\title{
Mira variables in the OGLE bulge fields ${ }^{\star}, \star \star$
}

\author{
M. A. T. Groenewegen ${ }^{1}$ and J. A. D. L. Blommaert ${ }^{1,2}$ \\ 1 Instituut voor Sterrenkunde, KU Leuven, Celestijnenlaan 200B, 3001 Leuven, Belgium \\ e-mail: groen@ster.kuleuven.ac.be \\ 2 Sterrenkundig Observatorium, Universiteit Gent, Krijgslaan 281-S9, 9000 Gent, Belgium
}

Received 25 March 2005 / Accepted 9 June 2005

\section{ABSTRACT}

The 222000 I-band light curves of variable stars detected by the OGLE-II survey in the direction of the Galactic Bulge have been fitted and also correlated with the DENIS and 2MASS all-sky release databases and with lists of known objects. Lightcurves and the results of the lightcurve fitting (periods and amplitudes) and DENIS and 2MASS data are presented for 2691 objects with $I$-band semi-amplitude larger than 0.45 mag, corresponding to classical Mira variables. That the Mira period distribution of 6 fields at similar longitude but spanning latitudes from -1.2 to -5.8 are statistically indistinguishable indicates similar populations with initial masses of $1.5-2 M_{\odot}$, corresponding to ages of $1-3$ Gyr. A field at similar longitude at $b=-0.05$ from Glass et al. (2001, MNRAS, 321, 77; erratum: 2002, MNRAS, 336, 1390) does show a significantly different period distribution, indicating the presence of a younger population of 2.5-3 $M_{\odot}$ and ages below 1 Gyr. The $K$-band period-luminosity relation is presented for the whole sample and for sub-fields. The zero point depends on Galactic longitude. Simulations are carried out to show that the observed dependence of the zero point with $l$, and the number of stars per field are naturally explained using the model of disk and bulge stars of Binney et al. (1997, MNRAS, 288, 365), for a viewing angle (major-axis Bar - axis perpendicular to the line-of-sight to the Galactic Centre) of $43 \pm 17$ degrees. The simulations also show that biases in the observed zero point are small, $<0.02 \mathrm{mag}$. A comparison is made with similar objects in the Magellanic Clouds. The slope of the $P L$-relation in the Bulge and the MCs agree within the errorbars. Assuming the zero point does not depend on metallicity, a distance modulus difference of 3.72 between Bulge and LMC is derived. This implies a LMC DM of 18.21 for an assumed distance to the Galactic Centre (GC) of $7.9 \mathrm{kpc}$, or, assuming a LMC DM of 18.50, a distance to the GC of $9.0 \mathrm{kpc}$. From the results in Groenewegen (2004, A\&A, 425, 595) it is found for carbon-rich Miras that the $P L$-relation implies a relative SMC-LMC DM of 0.38 , assuming no metallicity dependence. This is somewhat smaller than the often quoted value near 0.50 . Following theoretical work by Wood (1990, in From Miras to Planetary Nebulae, ed. M. O. Mennessier, \& A. Omont (Gif-sur-Yvette: Éditions Frontières), 67) a metallicity term of the form $M_{\mathrm{K}} \sim \beta \log Z$ is introduced. If a relative SMC-LMC DM of 0.50 is imposed, $\beta=0.4$ is required, and for that value the distance to the GC becomes $8.6 \pm 0.7 \mathrm{kpc}$ (for a LMC DM of 18.50), within the errorbar of the geometric determination of $7.9 \pm 0.4 \mathrm{kpc}$. An independent estimate leads to a distance estimate to the GC of $8.8 \pm 0.4 \mathrm{kpc}$.

Key words. stars: AGB and post-AGB - Galaxy: bulge - Galaxy: center

\section{Introduction}

In the course of the micro-lensing surveys in the 1990's the monitoring of the Small and Large Magellanic Clouds has revealed an amazing number and variety of variable stars. A big impact was felt and is being felt in all areas of variable star research, like Cepheids and RR Lyrae stars. Also in the area of variability in red variables (RVs) and AGB stars there has been remarkable progress. Wood et al. (1999) and Wood (2000) were the first to identify and label different sequences "ABC" thought to represent the classical Mira sequence ("C") and overtone pulsators ("A, B"), and sequence "D" which is not yet satisfactorily explained (Olivier \& Wood 2003;

${ }^{\star}$ Full Table 1 is available in electronic form at the CDS via anonymous ftp to cdsarc.u-strasbg.fr (130.79.128.5) or via http://cdsweb.u-strasbg.fr/cgi-bin/qcat?]/A+A/443/143

$\star \star$ Full Fig. 1, Full Table 2 and Appendices A-C are only available in electronic form at http://www. edpsciences.org
Wood et al. 2004. Stars on this last sequence are referred to as having Long Secondary Periods - LSPs). This view has been subsequently confirmed and expanded upon by Noda et al. (2002), Lebzelter et al. (2002), Cioni et al. (2003), Ita et al. (2004), Kiss \& Bedding (2003, 2004), and Fraser et al. (2005). These works differ in the source of the variability data (MACHO, OGLE, EROS, MOA), area (SMC or LMC), associated infrared data (Siding Spring $2.3 \mathrm{~m}$, DENIS, 2MASS, SIRIUS), and selection on pulsation amplitude or infrared colours. In a recent paper, Groenewegen (2004, hereafter G04) analysed the OGLE data in the SMC and LMC, and correlated these sources with the DENIS and 2MASS surveys. The paper discussed the variability properties of three samples: about 2300 spectroscopically confirmed AGB stars, around 400 previously known LPV variables, and about 570 candidate dust-obscured AGB stars.

The present paper is an extension of the analysis in G04 to the OGLE data in the direction of the Galactic Bulge (GB). For this area of the sky, several papers also exist that use the results 
of the micro-lensing surveys and have extended previous classical works on Bulge variable stars, like those of Lloyd Evans (1976), Glass \& Feast (1982), Whitelock et al. (1991), Glass et al. (1995, hereafter GWCF), Alard et al. (1996), and Glass et al. (2001).

Alard et al. (2001, hereafter ABC01) correlate ISOGAL sources within the NGC 6522 and Sgr I Baade windows with the MACHO database and present a list of 332 stars with complete 4-band $V, R$, and [7], [15] mag. Schultheis \& Glass (2001) extended Alard et al. by also considering the DENIS and 2MASS data in those fields in general and for the variables in particular. Glass \& Schultheis (2002, hereafter GS02) investigate a sample of $174 \mathrm{M}$-giants in the NGC 6522 Baade window and correlated them with DENIS ISOGAL and MACHO. Many stars of spectral type M 5 and all M 6 and later show variation, whereas subtypes M 1-M 4 do not (see also Glass et al. 1999).

Glass \& Schultheis (2003, hereafter GS03) investigated the variable stars in the NGC 6522 Baade's window using MACHO data, and also used DENIS IR data. Of the 1661 selected stars 1085 were found to be variable. They present $K$-band $P L$-relations for sequences "ABCD". Wray et al. (2004) investigated small amplitude red giants variables in a sub-set of 33 OGLE fields. They identified two groups that seem to correspond to groups "A-" and "B-" in Ita et al. (2004; also see G04).

In our paper we describe results on Mira variables selected from oGLE Bulge fields. The paper is structured as follows. In Sect. 2 the OGLE, 2MASS and DENIS surveys are described. In Sect. 3 the model for the lightcurve analysis is briefly presented. In the rest of the paper, different results are described. The Period-Luminosity diagram is discussed in Sect. 5. A description of the Mira population with respect to the overall bulge population is given in Sect. 8. In Sect. 9 we show that the Miras are distributed in a bar-like structure and give the orientation. In the final section we give the distance to the Galactic Centre, based on the period-luminosity relation.

\section{The data sets}

The OGLE-II micro-lensing experiment observed forty-nine fields in the direction of the GB. Each field is $14.2^{\prime} \times 57^{\prime}$ and was observed in $B V I$, with an absolute photometric accuracy of 0.01-0.02 mag (Udalski et al. 2002). Table 4 lists the galactic coordinates of the field centers and the total number of sources detected in these fields.

Wozniak et al. (2002) present a catalog of about 222000 variable objects based on the OGLE observations covering 1997-1999 and apply the difference image analysis (DIA) technique on the $I$-band data. The data files containing the $I$-band data of the candidate variable stars was downloaded from the OGLE homepage (http://sirius.astrouw.edu.pl/〜ogle/). According to Wozniak et al., the level of contamination by spurious detections is about $10 \%$, but we presume this level is much less at the brighter magnitudes of the LPVs considered here. Table 4 lists the number of detected variable stars per field (Wozniak et al. 2002).
The DENIS survey is a survey of the southern hemisphere in $I J K_{\mathrm{s}}$ (Epchtein et al. 1999). The second data release available through ViZier was used (The DENIS consortium, 2003). The 221801 OGLE objects were correlated on position using a $3^{\prime \prime}$ search radius and 59894 matches were found.

The 2MAss survey is an all-sky survey in the $J H K_{\mathrm{s}}$ nearinfrared bands. On March 25, 2003 the 2MASs team released the all-sky point source catalog (Cutri et al. 2003). The easiest way to check if a star is included in the 2MAss database is by uplinking a source table with coordinates to the 2MASS homepage. Such a table was prepared for the 221801 OGLE objects and correlated on position using a $3^{\prime \prime}$ radius. Data on 182361 objects were returned.

\section{Lightcurve analysis}

The model to analyse the lightcurves is described in detail in Appendices A-C in G04.

Briefly, a first code (see for details Appendix A in G04) sequentially read in the $I$-band data for the 222000 objects, determined periods through Fourier analysis, fit sine and cosine functions to the light curve through linear least-squares fitting, and made the final correlation with the pre-prepared DENIS and 2MASS source lists. All the relevant output quantities were written to file.

This file was read in by the second code (see for details Appendix B in G04). A further selection could be applied (typically on period, amplitude, and mean I-magnitude), multiple entries were filtered out (i.e. objects that appear in different OGLE fields), and a correlation made with pre-prepared lists of known non-LPVs and known LPVs or AGB stars. The output of the second code is a list with LPV candidates.

The third step (for details see Appendix C in G04) consisted of a visual inspection of the fits to the light curves of the candidate LPVs and a literature study through correlation with the SIMBAD database. Non-LPVs were removed, and sometimes the fitting was redone. The final list of LPV candidates was compiled.

Details on the small changes in the codes w.r.t. the implementation in G04 are given in Appendix A of the present paper.

\section{Comparison of the datasets}

\subsection{Astrometry}

As in G04, correlation between the OGLE objects and known LPVs and AGB stars, and known non-LPVs, was actually done in 2 steps. In the first step the correlation was made (for a $3^{\prime \prime}$ search radius), and the differences and spread in $\triangle \mathrm{RA} \cos (\delta)$ and $\Delta \delta$ determined. These mean offsets were then applied in most cases to make the final cross-correlation, and this usually increased the number of matches. The results are listed in Table 3.

\subsection{Photometry}

As in G04, a comparison was made between the (mean) OGLE I and the (single-epoch) DENIS $I$, and between the (single-epoch) 
Table 1. First entries in the electronically available table, which lists: OGLE-field and number, the three fitted periods with errors and amplitude (0.00 means no fit), mean $I_{\text {ogle }}$, associated DENIS $I J K$ photometry with errors, and associated 2MASS $J H K$ photometry with errors (99.9 and 9.99 means no association, or no value).

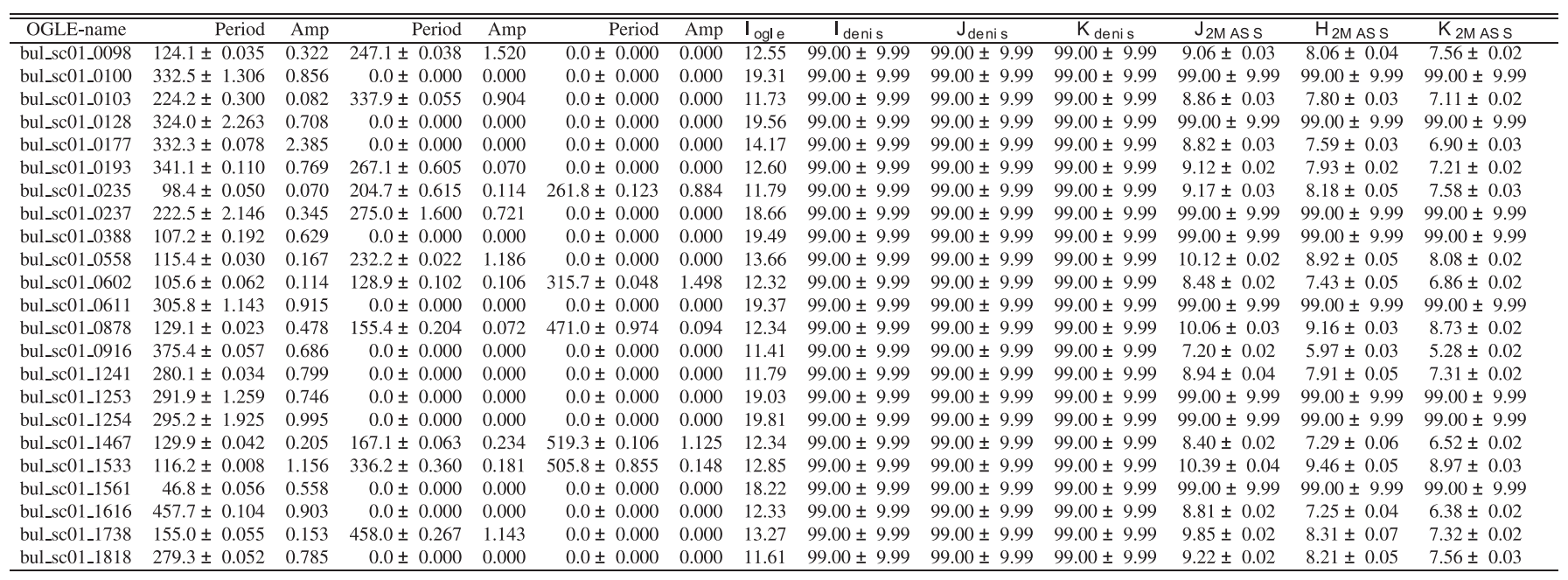

DENIS $J K$ and the (single-epoch) 2MASS $J K$ magnitudes. This was done by selecting those objects with an amplitude in the I-band of $<0.05 \mathrm{mag}$.

Figure 2 shows the final results when offsets $I$ (denisogle $)=-0.03, J($ denis -2 mass $)=-0.02$, and $K($ denis -2 mass $)=$ -0.03 are applied. The latter values are consistent with those derived in OOSO3 based on the 2MASS second incremental data release who found $J$ (denis-2mass) $=-0.02 \pm 0.09$, and $K($ denis -2 mass $)=-0.00 \pm 0.07$.

\section{Period-luminosity relations}

The full machinery outlined in Sect. 3 was performed. As in G04, all derived periods are given in Table 1 and are shown in Fig. 1. The present paper discusses only objects which have at least one period with an I-band amplitude larger than $0.45 \mathrm{mag}^{1}$, i.e. classical Mira variables (e.g. Hughes 1989). After visual inspection of the lightcurves, a sample of 2691 such objects remain. The number of objects per field is listed in the last column of Table 4.

Table 1 lists the stars in the sample, the fitted periods with errors and amplitudes, and the DENIS and 2MASS photometry of the associated sources. Table 2 lists alternative names and references from the literature. Figure 1 presents lightcurves and their fits.

Magnitudes are de-reddened using the $A_{\mathrm{V}}$ values that correspond to the respective OGLE field taken from Sumi (2004; and $A_{\mathrm{V}}=6.0$ for the field 44 that they do not discuss), selective reddenings of $A_{\mathrm{I}} / A_{\mathrm{V}}=0.49, A_{\mathrm{J}} / A_{\mathrm{V}}=0.27, A_{\mathrm{H}} / A_{\mathrm{V}}=0.20$, $A_{\mathrm{K}} / A_{\mathrm{V}}=0.12$ (Draine 2003), and implicitly assuming that all objects suffer this reddening value (i.e. ignoring differential reddening within a field, and ignoring that foreground and background objects would suffer a different reddening). Sumi's method is based on the absolute magnitude of the Red Clump

1 The amplitude, $A$, is used in the mathematical sense in the present paper, $y=A \sin x$. The peak-to-peak amplitude is 0.90 mag. giants and is absolute calibrated using the $(V-K)$ colours of 20 RR Lyrae stars in Baade's window. Popowski et al. (2003) present an extinction map (over 9000 resolution elements of $4 \times 4$ arcmin size) towards the GB based on MACHO $V, R$ photometry, under the assumption that colour-magnitude diagrams would look similar in the absence of extinction. For the centre of the OGLE fields it was checked if there was a tile in the Popowski et al. set within 0.05 degrees distance. For those, the value of the visual extinction has been listed next to the value in Sumi in Table 4. The rms difference $A_{\mathrm{V}}$ for the 21 field with values from both references is 0.18 . Finally, Schultheis et al. (1999b) present a reddening map for the inner GB comparing DENIS $J, K$ photometry to isochrones. Table 4 lists the values they find for two OGLE fields: SC44 which was not considered by Sumi (2004), and SC5 for which Sumi derive a larger $A_{\mathrm{V}}$ than Schultheis et al.: 5.73 versus 4.13 .

In the further discussion we only use periods that fulfil the following conditions are used in the calculations (with $\Delta P$ the error in the period): $\Delta P / P<0.01$ for $P<500^{\mathrm{d}} ; \Delta P<5^{\mathrm{d}}$ for $500^{\mathrm{d}}<P<800^{\mathrm{d}}$ and $\Delta P<1.5^{\mathrm{d}}$ for $P>800^{\mathrm{d}}$. The latter constraint was necessary because the long periods become comparable to the length of the dataset.

Figure 3 shows the $K$-band $P L$-relation for all periods which have an $I$-band amplitude larger than 0.45 mag and $(J-K)_{0}<2.0$ among the 2691 stars. The cut in $(J-K)$ colour is needed to prevent the $K$-magnitude being affected by circumstellar extinction, as shown in G04. Like G04, the $K$-magnitude is on the 2MASs system, and is the average of the DENIS and 2MASS photometry. In particular, if both DENIS and 2MASS $K$-band data are available, the DENIS data point is corrected as explained above (i.e. 0.03 mag added), and averaged with the 2MASs data point. This should take out some of the scatter in the $P L$-diagram, as the effect of the variability in the $K$-band is reduced. If only DENIS is available, the corrected value was used. In the left-hand panel the boundaries of the boxes "A-, A+, B-, B+, C, D" were taken from G04, but shifted by -4.0 to account for the approximate difference 

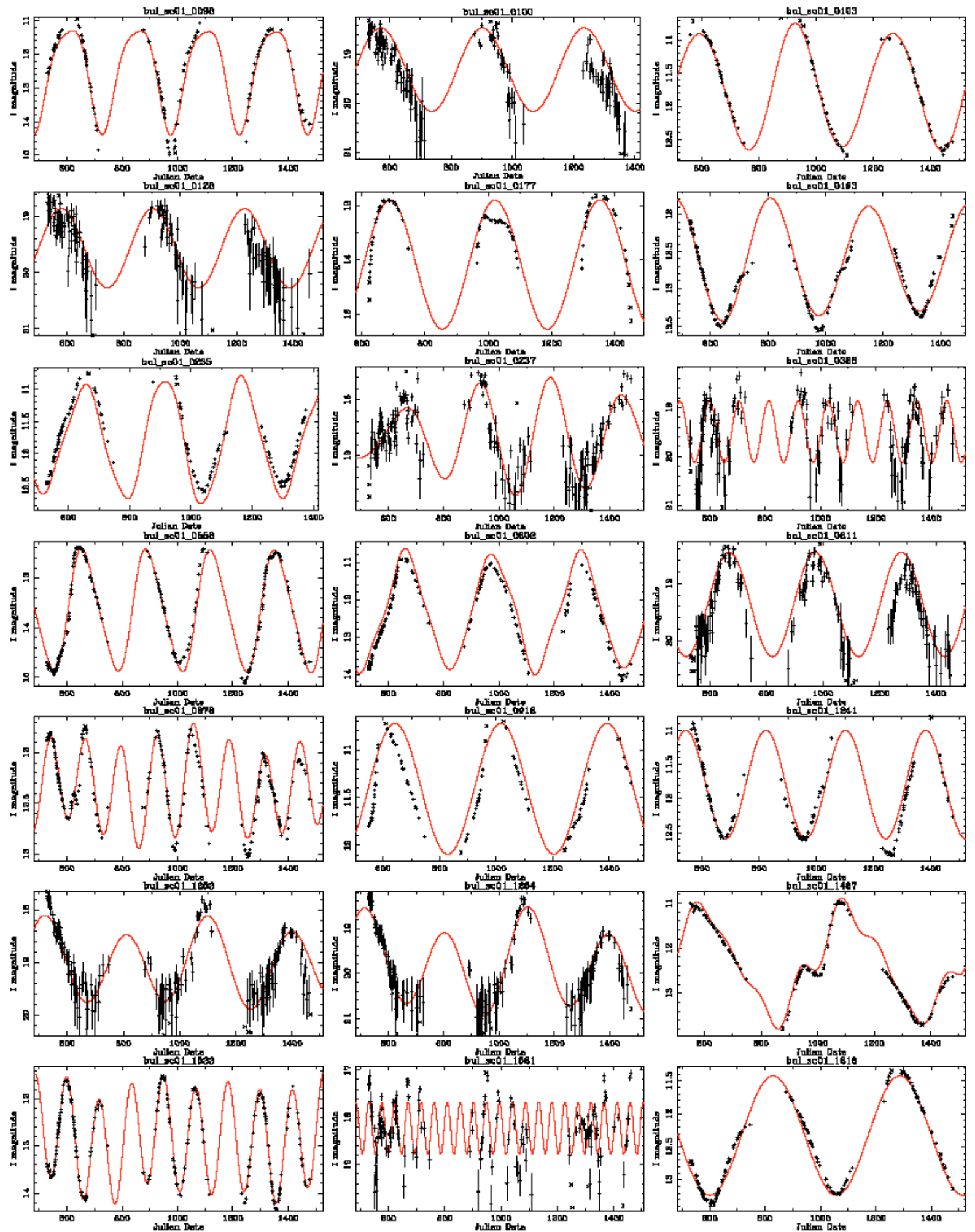

Fig. 1. First entries of electronically available figure with all lightcurves. The fit is indicated by the (red) solid line. Crosses indicate data points not included in the fit.

in distance modulus (DM), as, e.g., follows from the recent determination of $7.9 \pm 0.4 \mathrm{kpc}$ (Eisenhauer et al. 2003) for the distance to the GC, and 18.50 for the DM to the LMC (e.g. recent reviews by Walker 2003; Feast 2004a).

There is a reasonably well-defined sequence in Box " $\mathrm{C}$ ", but when compared to the similar figure for the SMC and LMC in G04 (his Fig. 3) some differences can be noted as well. For the present Bulge sample there are a few objects located in Box "B+", and many in Box "D". In the SMC and LMC for this cut in amplitude, there are none in Box " $\mathrm{B}+$ " and few in " $\mathrm{D}$ ". Several issues may play a role. Applying a certain cut in amplitude may sample slightly different variables in SMC, LMC, 

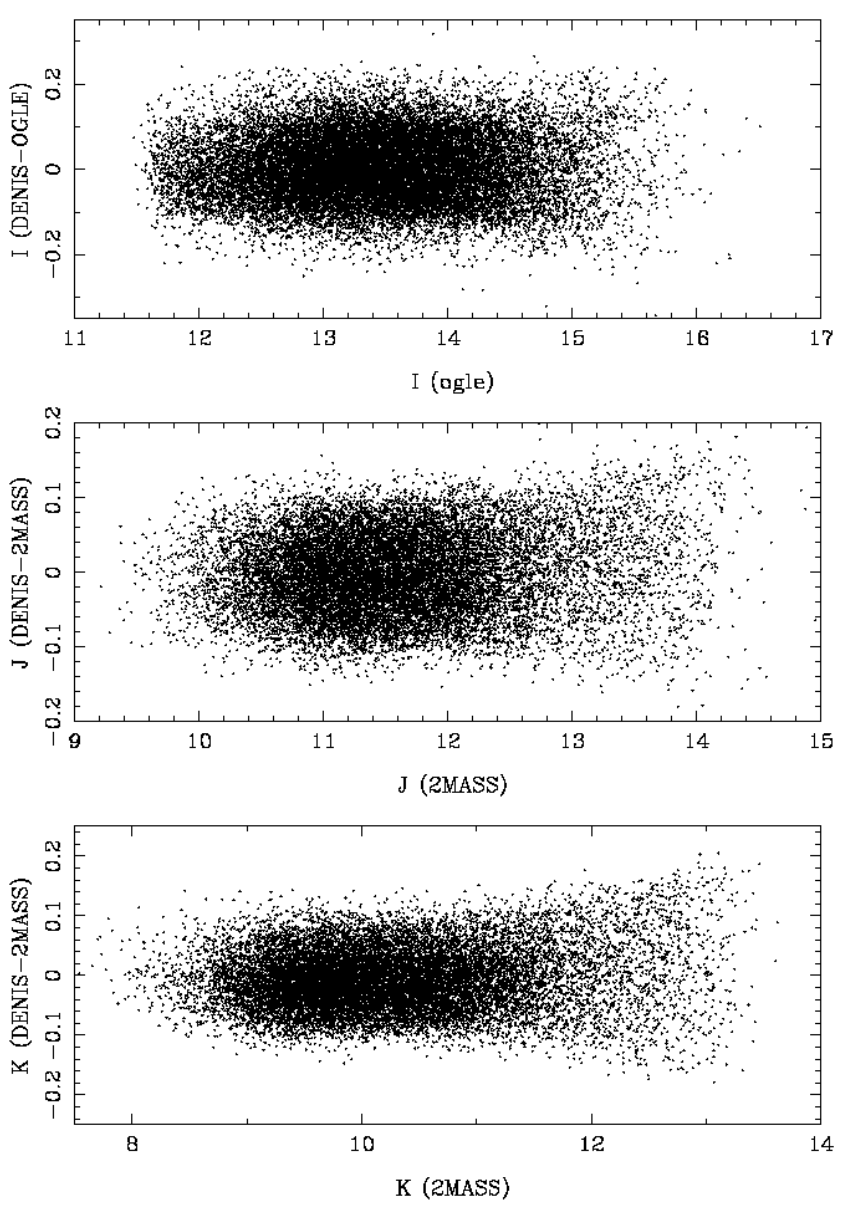

Fig. 2. Difference in photometry, after the following offsets were applied: $I($ denis-ogle $)=-0.03, J($ denis- 2 mass $)=-0.02, K($ denis2 mass $)=-0.03$.

and Bulge. Figure 3 in G04 clearly shows how lowering the cut in amplitude results in populating Box " $\mathrm{B}+$ " and then " $\mathrm{A}+$ ", and increases the number of objects in " $D$ ". Another effect is the possible contribution of objects in the foreground and background of the Bulge, the depth of the Bulge, and finally, the orientation of the Bar, as the OGLE fields span 20 degrees in longitude (this last effect will be discussed later). Finally, the difference in DM may be different from the adopted value of 4.0 .

To verify if the objects in Box "D" actually show LSP, they were all visually inspected. Few have a LSP, in agreement with the finding for LMC and SMC (for amplitudes $>0.45 \mathrm{mag}$ ). This would call for enlargement of Box "C" to properly sample the $P L$-relation of the large amplitude (Mira) variables. To define this enlarged box, the $P L$-relation was inspected for each field independently. The right-hand panel in Fig. 3 shows the finally adopted boundaries of Box "C", which implies that Box "D" has contracted. Stars inside this redefined Box will be used to define the $P L$-relation. The $K$-band $P L$-relation is determined to be

$m_{\mathrm{K}}=(-3.37 \pm 0.09) \log P+(15.44 \pm 0.21)$

with an rms of 0.42 and based on 1292 stars, as shown in Fig. 3. The value of the slope is consistent with the median value when
Table 2. First entries in the electronically available table, which list: OGLE-field and number, other names, spectral type, and references. ISOGAL sources from the official catalog (OGA03) have the prefix "ISOGAL". ISOGAL sources studied in OOS03 have the prefix "OOS03". References in Col. 4 are given in the bibliography of the main text.

\begin{tabular}{|c|c|c|c|}
\hline " OGLE-name & Other name & Chemical type & Remarks \\
\hline bul_sc01_0098 & V 4713 Sgr & & \\
\hline bul_sc01_0100 & & & \\
\hline bul_sc01_0103 & & & \\
\hline bul_sc01_0128 & & & \\
\hline bul_sc01_0177 & & & \\
\hline bul_sc01_0193 & & & \\
\hline bul_sc01_0235 & & & \\
\hline bul_sc01_0237 & & & \\
\hline bul_sc01_0388 & & & \\
\hline bul_sc01_0558 & BW6_V1_MISC & & \\
\hline bul_sc01_0602 & & & \\
\hline bul_sc01_0611 & & & \\
\hline bul_sc01_0878 & & & \\
\hline bul_sc01_0916 & & & \\
\hline bul_sc01_1241 & & & \\
\hline bul_sc01_1253 & & & \\
\hline bul_sc01_1254 & & & \\
\hline bul_sc01_1467 & BW5_V1_MISC & & \\
\hline bul_sc01_1533 & & & \\
\hline bul_sc01_1561 & & & \\
\hline bul_sc01_1616 & & & \\
\hline bul_sc01_1738 & BW5_V4_MISC & & \\
\hline bul_sc01_1818 & & & \\
\hline
\end{tabular}

the $P L$-relation is determined for all fields individually. For reference, fitting all stars in Fig. 3, for a fixed slope of -3.37 results in a $\mathrm{ZP}$ of $15.47 \pm 0.55$.

\section{Historical versus current periods}

Table 5 compares the period derived in the present paper (in case of multiple periods the one with the largest amplitude) with values derived in the literature. There are three cases where a previously determined period may be a harmonic of the present period, but overall there is good agreement between periods. In the 12 cases where there is a period available from LE76 (with the photographic plates taken between 1969 and 1971 , hence 28 years of time difference with OGLE), there is no clear case for a star that has changed period. By comparison, G04 found that about $8 \%$ of LMC variables changed their period by more than $10 \%$ over about a 17 -year timespan. To find 0 out of 12 in the present sample is consistent with this.

\section{Colour-colour diagrams}

The 2MASS and DENIS Colour-colour and colour-magnitude diagrams are shown in Fig. 4, together with that of spectroscopically confirmed M-stars in the LMC (see also Fig. 12 in G04). There appear to be more of the redder stars in the Bulge sample, but this is likely due to under representation in the LMC sample as this was restricted to spectroscopically known 
Table 3. Comparison of coordinates and number of positional matches, before and after a correction was applied.

\begin{tabular}{|c|c|c|c|c|c|c|c|}
\hline $\begin{array}{l}\text { OGLE } \\
\text {-(other) }\end{array}$ & 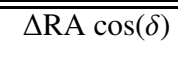 & 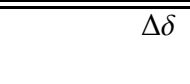 & $\bar{N}$ & 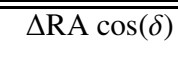 & 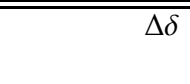 & $\bar{N}$ & Remark \\
\hline IRAS & $0.14 \pm 0.94$ & $0.50 \pm 1.44$ & 80 & & & & IRAS sources, not corrected \\
\hline OGA03 & $-0.80 \pm 0.85$ & $-0.16 \pm 0.89$ & 1309 & $-0.11 \pm 0.82$ & $-0.02 \pm 0.90$ & 1319 & ISOGAL sources from Omont et al. (2003; OGA03) \\
\hline OOS03 & $-0.80 \pm 0.88$ & $-0.30 \pm 0.95$ & 800 & $-0.10 \pm 0.86$ & $-0.03 \pm 0.99$ & 817 & ISOGAL sources from Ojha et al. (2003; OOS03) \\
\hline GSO2 & $-0.02 \pm 0.58$ & $0.30 \pm 0.61$ & 79 & $-0.04 \pm 0.67$ & $0.00 \pm 0.61$ & 80 & MACHO sources from Glass \& Schultheis (2002; GS02) \\
\hline $\mathrm{ABC} 01$ & $-0.82 \pm 0.98$ & $-0.70 \pm 0.96$ & 293 & $-0.17 \pm 1.08$ & $-0.12 \pm 1.01$ & 320 & ISOGAL sources from Alard et al. (2001; ABC01) \\
\hline OGLE-I & $-0.08 \pm 0.70$ & $-0.18 \pm 0.44$ & 728 & & & & OGLE-I sources, not corrected \\
\hline BMB & $-0.04 \pm 0.70$ & $-0.52 \pm 0.90$ & 282 & $+0.10 \pm 0.79$ & $-0.08 \pm 0.91$ & 286 & sources from Blanco et al. (1984; BMB) \\
\hline TLE & $-0.08 \pm 0.84$ & $-0.25 \pm 1.43$ & 21 & & & & sources from Lloyd-Evans (1976), not corrected \\
\hline B84 & $-0.46 \pm 0.75$ & $0.50 \pm 0.97$ & 61 & $-0.02 \pm 0.76$ & $0.08 \pm 1.05$ & 63 & sources from Blanco (1984; B84) \\
\hline
\end{tabular}
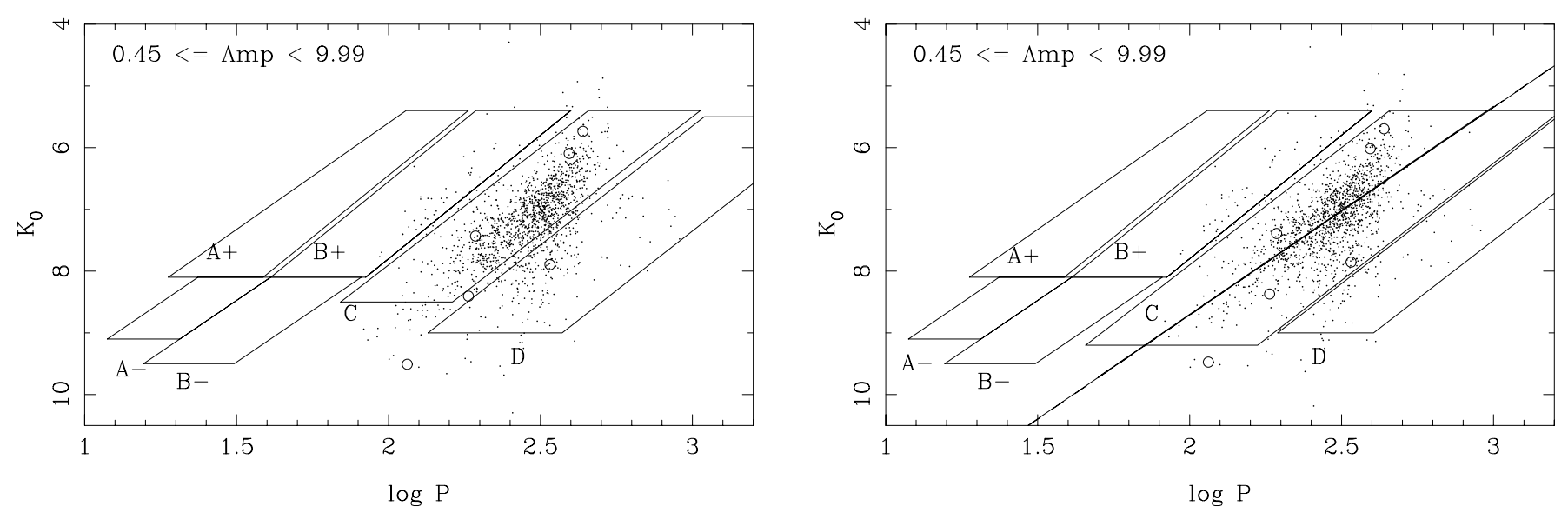

Fig. 3. $K$-band $P L$-relation for periods with an $I$-band amplitude larger than 0.45 mag and $(J-K)_{0}<2.0$. Left panel: sequences/Boxes "A+, A-, B+, B-, C, D" taken from G04 for the LMC and shifted by -4.0 in distance modulus. Right panel: enlarged Box "C" (and contracted Box "D",) to define the region for which the $P L$-relations will be computed. Only periods from Table 1 that fulfil $\Delta P / P<0.01$ for $P<500^{\mathrm{d}}$; $\Delta P<5^{\mathrm{d}}$ for $500^{\mathrm{d}}<P<800^{\mathrm{d}}$ and $\Delta P<1.5^{\mathrm{d}}$ for $P>800^{\mathrm{d}}$ are plotted and used in the analysis. Known M-stars are indicated by open circles. The line in the right-hand panel indicates the $P L$-relation of Eq. (1).

M-stars (i.e. in general optically selected). The sample of candidate infrared-selected AGB stars in the LMC (Fig. 12 in G04) does cover the $(I-K)$ and $(J-K)$ colour range observed in the Bulge).

The other main difference is that the Bulge stars are redder by $\sim 2$ mag in $(I-J)_{0}$ than both LMC and SMC stars, as was also shown by Lebzelter et al. (2002) in a comparison of LMC and Bulge variable stars. As the diagrams involving $J, H, K$ colours appear similar, it seems that this difference in $(I-J)$ must be due to a difference in $I$. The $I$-band measurements of $\mathrm{M}$ stars is strongly affected by the $\mathrm{TiO}$ and $\mathrm{VO}$ molecular absorption features (Lançon \& Wood 2000). It is expected that for larger metallicities these lines will be stronger (Schultheis et al. 1999a), which will lead to redder $(I-J)$ colours.

The bullets connected by a line in the Bulge DENIS $(I-J)-$ $(J-K)$ colour-colour diagram are the average colours of $\mathrm{M} 1$, M 2, .., M 6, M 6.5, M 7, M 8 giants in the NGC 6522 Baade's window (Blanco 1986, GS02). There is a spread of typically 0.3-0.5 mag in $(I-J)$ and 0.2-0.3 mag in $(J-K)$ around these means, and there is only $1 \mathrm{M} 8$ giant in their sample. The colours of the Miras follow those of normal giants well until M 6.5, when the Miras become redder in $(I-J)$. There are also stars redder in $(J-K)$ than the single M 8 star in the sample of
GS02, indicating either the presence of later spectral types or the onset of circumstellar reddening.

The conclusion of GS02 that "Many M 5 and all stars M 6 and later show variation, whereas subtypes (M 1-M 4) do not", is confirmed here, as there are essentially no objects located in the region of the DENIS $(I-J)-(J-K)$ colour-colour diagram occupied by spectral types of M 4 and earlier.

\section{Mira bulge population as function of latitude}

Figure 5 shows the period distribution of Miras in Box " $\mathrm{C}$ ". A distinction is made between all Miras and those with $(J-$ $K)_{0}<2.0$ (dashed histograms). The latter selection minimises any influence of circumstellar extinction. For comparison, the period distribution of LMC and SMC Miras is also shown ${ }^{2}$. The Kolmogorov-Smirnov (KS) test was performed to indicate that the probability that the period distributions of Bulge-LMC, Bulge-SMC, LMC-SMC are the same for all stars (those with $\left.(J-K)_{0}<2\right)$ is, respectively $0.36\left(10^{-8}\right), 0.05(0.31)$, and 0.05 $(0.05)$.

\footnotetext{
${ }^{2}$ Derived following the implementation of the code and definition of the "boxes" as in G04, and applying the same selection criteria as in the present paper, i.e. $I$-amplitude larger than $0.45 \mathrm{mag}$.
} 
Table 4. Properties of the OGLE-fields.

\begin{tabular}{|c|c|c|c|c|c|c|}
\hline BUL_SC & $l$ & $b$ & Total $^{(a)}$ & Variable $^{(b)}$ & $A_{\mathrm{V}}{ }^{(c)}$ & $\operatorname{LPV}^{(d)}$ \\
\hline 1 & 1.08 & -3.62 & 730 & 4597 & $1.68 / 1.49$ & 42 \\
\hline 2 & 2.23 & -3.46 & 803 & 5279 & $1.55 / 1.65$ & 48 \\
\hline 3 & 0.11 & -1.93 & 806 & 8393 & 2.89 & 115 \\
\hline 4 & 0.43 & -2.01 & 774 & 9096 & $2.59 / 2.94$ & 86 \\
\hline 5 & -0.23 & -1.33 & 434 & 7257 & $5.73 /-/ 4.13$ & 118 \\
\hline 6 & -0.25 & -5.70 & 514 & 3211 & 1.37 & 47 \\
\hline 7 & -0.14 & -5.91 & 463 & 1618 & $1.33 / 1.28$ & 21 \\
\hline 8 & 10.48 & -3.78 & 402 & 2331 & 2.14 & 8 \\
\hline 9 & 10.59 & -3.98 & 330 & 1847 & 2.08 & 21 \\
\hline 10 & 9.64 & -3.44 & 458 & 2499 & 2.23 & 16 \\
\hline 11 & 9.74 & -3.64 & 426 & 2256 & 2.27 & 18 \\
\hline 12 & 7.80 & -3.37 & 535 & 3476 & $2.29 / 2.20$ & 33 \\
\hline 13 & 7.91 & -3.58 & 570 & 3084 & $2.06 / 1.82$ & 21 \\
\hline 14 & 5.23 & 2.81 & 619 & 4051 & 2.49 & 51 \\
\hline 15 & 5.38 & 2.63 & 601 & 3853 & 2.77 & 71 \\
\hline 16 & 5.10 & -3.29 & 700 & 4802 & $2.15 / 2.23$ & 45 \\
\hline 17 & 5.28 & -3.45 & 687 & 4690 & $1.94 / 2.29$ & 167 \\
\hline 18 & 3.97 & -3.14 & 749 & 5805 & 1.83 & 55 \\
\hline 19 & 4.08 & -3.35 & 732 & 5255 & 2.74 & 51 \\
\hline 20 & 1.68 & -2.47 & 785 & 5910 & $1.94 / 2.02$ & 64 \\
\hline 21 & 1.80 & -2.66 & 883 & 7449 & $1.83 / 1.78$ & 60 \\
\hline 22 & -0.26 & -2.95 & 715 & 5589 & 2.74 & 70 \\
\hline 23 & -0.50 & -3.36 & 723 & 4815 & 2.70 & 60 \\
\hline 24 & -2.44 & -3.36 & 612 & 4304 & 2.52 & 56 \\
\hline 25 & -2.32 & -3.56 & 622 & 3046 & 2.34 & 61 \\
\hline 26 & -4.90 & -3.37 & 728 & 4713 & 1.86 & 39 \\
\hline 27 & -4.92 & -3.65 & 691 & 3691 & 1.69 & 46 \\
\hline 28 & -6.76 & -4.43 & 406 & 1472 & 1.64 & 16 \\
\hline 29 & -6.64 & -4.62 & 492 & 2398 & 1.53 & 36 \\
\hline 30 & 1.94 & -2.84 & 762 & 6893 & $1.91 / 1.78$ & 50 \\
\hline 31 & 2.23 & -2.94 & 790 & 4789 & $1.81 / 1.74$ & 59 \\
\hline 32 & 2.34 & -3.14 & 797 & 5007 & $1.61 / 1.82$ & 54 \\
\hline 33 & 2.35 & -3.66 & 739 & 4590 & $1.70 / 1.82$ & 105 \\
\hline 34 & 1.35 & -2.40 & 961 & 7953 & $2.52 / 2.32$ & 60 \\
\hline 35 & 3.05 & -3.00 & 771 & 5169 & $1.84 / 2.20$ & 39 \\
\hline 36 & 3.16 & -3.20 & 873 & 8805 & $1.62 / 1.52$ & 38 \\
\hline 37 & 0.00 & -1.74 & 664 & 8367 & 3.77 & 108 \\
\hline 38 & 0.97 & -3.42 & 710 & 5072 & $1.83 / 1.94$ & 57 \\
\hline 39 & 0.53 & -2.21 & 784 & 7338 & $2.63 / 2.70$ & 99 \\
\hline 40 & -2.99 & -3.14 & 631 & 4079 & 2.94 & 55 \\
\hline 41 & -2.78 & -3.27 & 603 & 4035 & 2.65 & 49 \\
\hline 42 & 4.48 & -3.38 & 601 & 4360 & 2.29 & 32 \\
\hline 43 & 0.37 & 2.95 & 474 & 3351 & 3.67 & 112 \\
\hline 44 & -0.43 & -1.19 & 319 & 7836 & $6.0 /-/ 6.00$ & 132 \\
\hline 45 & 0.98 & -3.94 & 627 & 2262 & $1.64 / 1.53$ & 32 \\
\hline 46 & 1.09 & -4.14 & 552 & 2057 & $1.71 / 1.65$ & 26 \\
\hline 47 & -11.19 & -2.60 & 301 & 1152 & 2.60 & 12 \\
\hline 48 & -11.07 & -2.78 & 287 & 973 & 2.35 & 12 \\
\hline 49 & -11.36 & -3.25 & 251 & 826 & 2.09 & 18 \\
\hline Total & & & 30490 & 221701 & & 2691 \\
\hline
\end{tabular}

(a) Total number of objects detected in the field. From Udalski et al. (2002), in units of $10^{3}$ objects. ${ }^{(b)}$ Total number of candidate variable stars. From Wozniak et al. (2002). ${ }^{(c)}$ Visual extinction. From Sumi (2004), except for SC44, where $A_{\mathrm{V}}=6.0$ has been adopted based on the proximity to SC5. The second value - when listed - comes from Popowski et al. (2003). The third value - when listed - comes from Schultheis et al. (1999b). ${ }^{(d)}$ Total number of LPVs.
Any difference, in particular between Bulge and LMC period distribution, is difficult to quantify further as this depends in a complicated way on the Star Formation History and evolutionary tracks ( $T_{\text {eff }}-$ Luminosity - Mass - metallicity). Regarding the period distribution of Bulge Miras as such, previous studies are limited to selected small fields (e.g. TLE, GWCF, Glass et al. 2001). Whitelock et al. (1991) present the period distribution of about 140 IRAS sources, but no direct comparison is possible because of the difference in the selection criteria of the samples.

Figure 6 shows the period distribution of selected fields with very similar longitudes that cover a range in latitudes (the stars with $(J-K)_{0}<2.0$ are again shown as dashed histogram). To add a field even closer to the GC than surveyed by OGLE the data in Glass et al. $(2001,2002)$ is considered on a field centred on $l=-0.05, b=-0.05$. They present the results of a $K$-band survey of $24 \times 24 \operatorname{arcmin}^{2}$ for LPVs down to $K \sim 12.0$. From the list of 409 stars, 14 were removed because of double entries, quality index of zero, uncertain or no listed period or amplitude. The coordinates were uploaded to the IPAC webserver, and 2MASs data within $2.5^{\prime \prime}$ was retrieved for these 395 stars to get information on the $(J-K)$ colour. As an additional check and to eliminate multiple stars within the search circle, it was verified that the single-epoch 2MASS $K$ magnitude is consistent with the mean $K$-magnitude and amplitude listed in Glass et al. For 345 stars 2MAss data is available. The magnitudes were corrected for interstellar reddening using the extinction map of the inner GB at $2^{\prime}$ resolution by Schultheis et al. (1999b). The extinction value of the nearest available grid point in this map was taken. The extinction values range between 18.5 and 30.4 with a mean of 24.7. The top panel in Fig. 6 lists 333 stars with $K>7$ (to eliminate 3 likely foreground objects) and $K$-band amplitude larger than 0.35 (to correspond roughly to the cut in I-band amplitude of $0.45 \mathrm{mag}$ ), and 88 (the histogram with slanted hatching), or 236 (dashed histogram) stars which also have $(J-K)_{0}<2.0$. The last sample is the one that results when the reddening values from Schultheis et al. are multiplied by 1.35 . They mention that the reddening may be underestimated in the direction of the GC because of $J$-band nondetections. For the one field in common, their value is a factor 1.3-1.4 smaller than derived by Sumi (2004). In addition, for their default reddening (the histogram with slanted hatching in Fig. 6) there would be many stars even at periods shorter than about 250 days that still would have $(J-K)_{0}>2.0$ which is not observed in the other fields. This could of course be real, but it is generally believed (e.g. Launhardt et al. 2002) that the population of low- and intermediate mass stars in the Nuclear Bulge (the inner about $30 \mathrm{pc}$ from the GC) and GB are similar, but that in the former there is an overabundance of $10^{7}-10^{8}$ year old stars. In this picture one would expect the period distributions to be similar at shorter periods, essentially independent of latitude. Therefore the period distribution of stars with $(J-K)_{0}<2.0$ for the increased reddening is adopted.

The KS test was performed on consecutive fields in latitude for the distributions based on the stars with $(J-K)_{0}<2.0$. It was found that the probability that the distributions are the same is $10^{-10}$ for the $b=-0.05 /-1.21$ fields, 0.50 for the $b=$ $-1.21 /-1.39$ fields, 0.80 for the $b=-1.39 /-1.81$, and $>0.99$ 
Table 5. Current period compared to values listed in the literature.

\begin{tabular}{lll}
\hline \hline OGLE-name & Periods & Remark \\
\hline bul_sc01_0558 & 232.2 (OGLE-II), 217.0, Mira (OGLE-I) & harmonic? \\
bul_sc01_1467 & 519.3 (OGLE-II), 265.0, Mira (OGLE-I) & harmonic? \\
bul_sc01_1738 & 458.0 (OGLE-II), 227.5, Mira (OGLE-I) & \\
bul_sc01_2079 & 524.7 (OGLE-II), 520.0, Mira (OGLE-I) & \\
bul_sc20_0832 & 245.9 (OGLE-II), 254.1: (ABC01), 235 (LE76), 336 (GWCF) & \\
bul_sc20_0975 & 167.7 (OGLE-II), 145.8 (ABC01) & harmonic? \\
bul_sc20_1189 & 220.0 (OGLE-II), 121.3 (ABC01) & \\
bul_sc20_1292 & 87.7 (OGLE-II), 88.1 (ABC01) & \\
bul_sc20_1761 & 231.6 (OGLE-II), 107.9 (ABC01), 240 (LE76), 235 (GWCF) & \\
bul_sc20_1826 & 331.7 (OGLE-II), 330: (LE76) & \\
bul_sc20_1928 & 297.6 (OGLE-II), 300 (LE76), 293 (GWCF) & \\
bul_sc20_2013 & 453.3 (OGLE-II), 474.2: (ABC01), 500:: (LE76), 480 (GWCF) & \\
bul_sc20_2269 & 409.3 (OGLE-II), 400: (LE76), 383 (GWCF) & \\
bul_sc20_2291 & 311.2 (OGLE-II), 306.9: (ABC01), 315 (LE76), 308 (GWCF) & \\
bul_sc34_3759 & 289.8 (OGLE-II), 293.8 (ABC01), 265 (LE76), 237 (GWCF) & \\
bul_sc45_0704 & 405.3 (OGLE-II), 430 (LE76), 467, Mira (OGLE-I), 413 (GS03) & \\
bul_sc45_1068 & 115.2 (OGLE-II), 115 (LE76), 116 (GCVS), 116 (GS03) & \\
bul_sc45_1586 & 193.3 (OGLE-II), 190 (LE76), 195 (GS03) & \\
bul_sc46_0866 & 322.1 (OGLE-II), 305 (LE76), 320 (GS03) & \\
bul_sc46_1163 & 343.7 (OGLE-II), 329.0, Mira (OGLE-I) & \\
\hline
\end{tabular}

2MASS
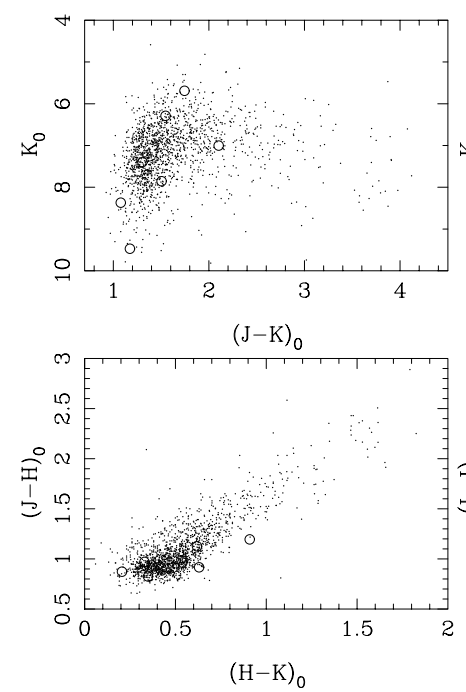

DENIS
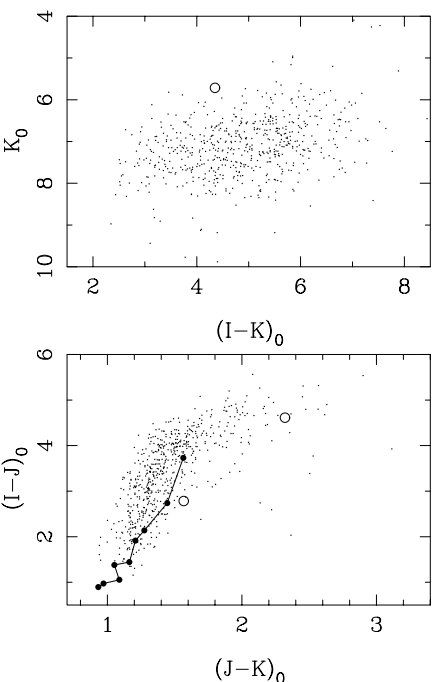

2MASS
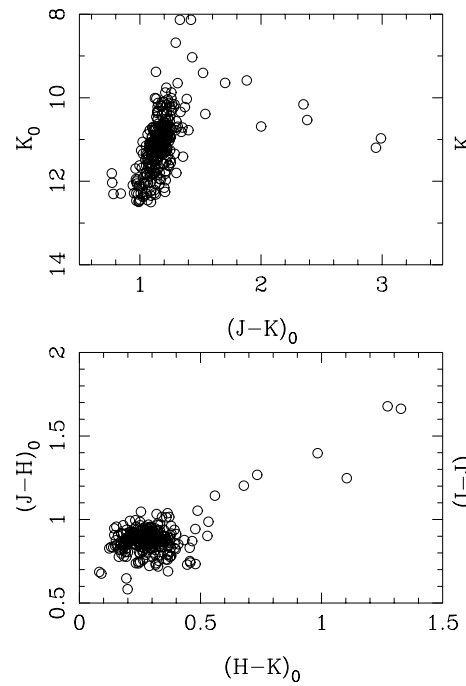

DENIS

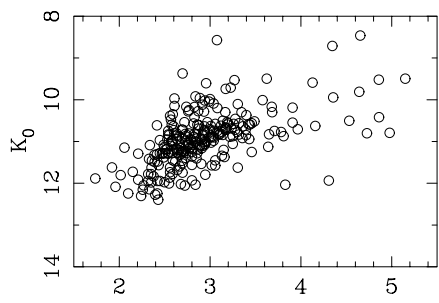

$(\mathrm{I}-\mathrm{K})_{0}$

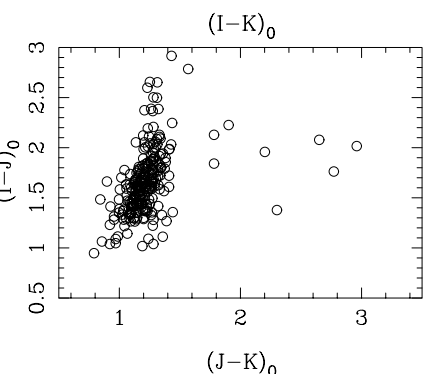

Fig. 4. Colour-magnitude and colour-colour diagrams using 2MASS and DENIS photometry for the Bulge stars (left), and spectroscopically confirmed M-stars in the LMC (right, from G04). The bullets connected by a line in the Bulge DENIS $(I-J)-(J-K)$ diagram are the average colours of M 1, M 2, .., M 6, M 6.5, M 7, M 8 giants in the NGC 6522 Baade's window (Blanco 1986; Glass \& Schultheis 2002).

for the fields at more negative latitudes. The conclusion is that the period distributions of the fields at and below -1.2 degree are statistically indistinguishable, but that the field at -0.05 latitude has a significantly different period distribution; the probability that this distribution is the same as the distribution of the combined 6 oGLE fields is $10^{-22}$. This conclusion is independent of the assumed reddening of the inner Bulge field, which influences how many stars will have $(J-K)_{0}<2.0$. For the default reddening of Schultheis et al., the probability that the distributions are the same for the $b=-0.05 /-1.21$ fields is still only 0.0033 . The difference in the period distributions is especially clear at longer periods. Of the 236 stars in the inner field with $(J-K)_{0}<2.061$ have $P>500$ days, while in the other fields this is 3 out of 367 .

The difference in period distribution might be due to an under representation of short period stars in the inner field. However, Fig. 4 in Glass et al. illustrates that the expected $K$-magnitudes at short periods are not fainter than the completeness limit of their survey. In fact, Glass et al. mention that they expect that the number of short-period Miras $(P<$ 250 days) is at least $75 \%$ complete. As a test, one-third of those stars with $P<250$ days were randomly duplicated and added 

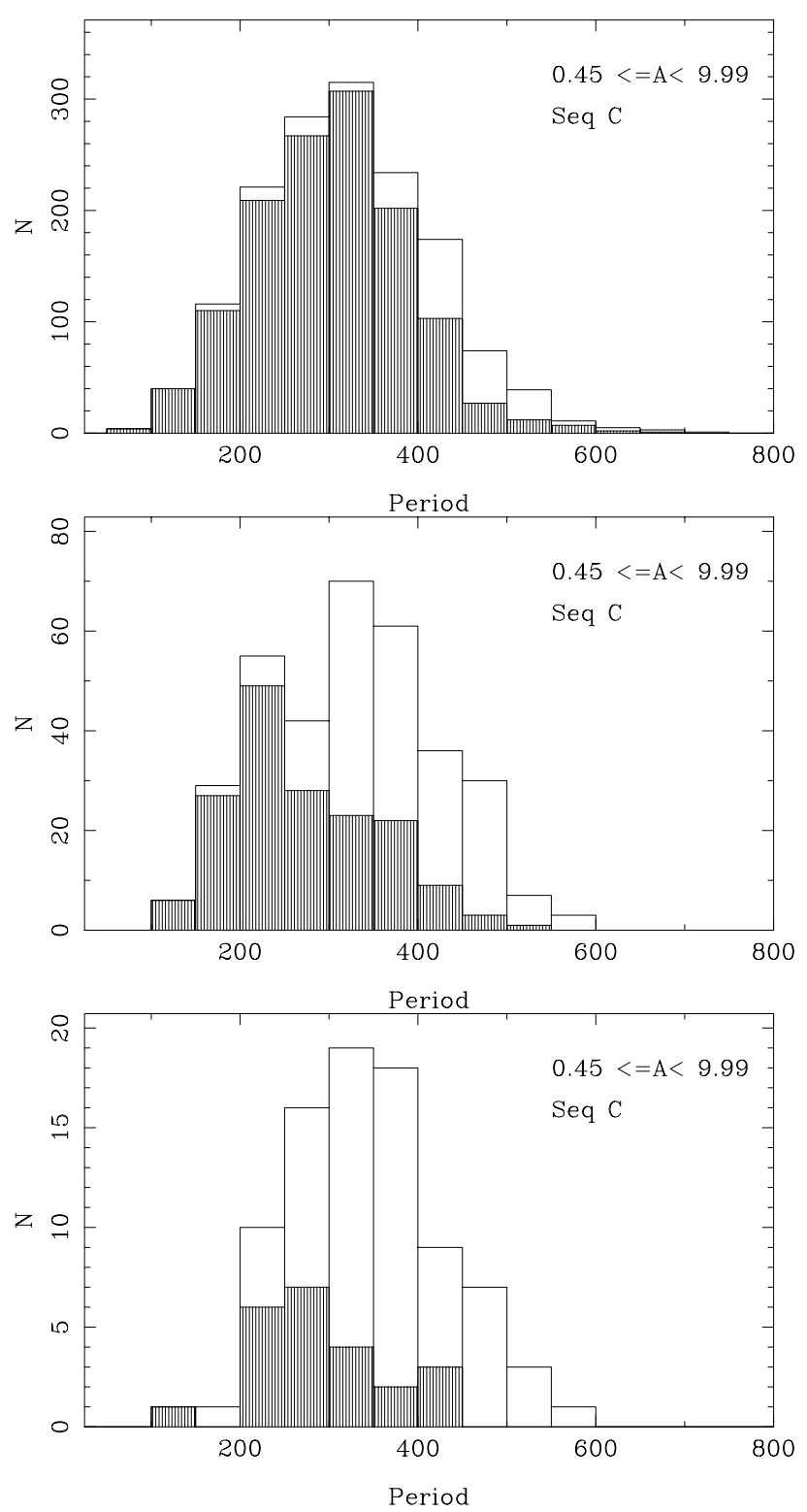

Fig. 5. Period distribution of large amplitude variables in box "C", for Bulge (top), LMC (middle), and SMC (bottom). For the shaded histogram only stars with $(J-K)_{0}<2.0$ have been included.

to the sample, and the KS test was repeated to find again a large difference between the period distribution of the field at -0.05 degrees and the other fields.

This difference is emphasised in Fig. 7 where the scaled period distribution of stars which have $(J-K)_{0}<2.0$ in the 5 fields between $b=-1.39^{\circ}$ and $b=-5.8^{\circ}$ has been subtracted from the inner field. The scaling was done in such a way that at shorter periods the two distributions would cancel at a level of $1 \sigma$ (based on Poisson errors). Even if the scaling is done in a slightly different way, the result is always very similar, in the sense that there is a significant $(>4 \sigma)$ overabundance of LPVs in the inner field between about 350 and 600 days.

The conclusion is that there is a significant population of LPVs with period $\gtrsim 500$ days present in the inner field, which remains barely present at latitude $-1.2^{\circ}$ and is absent for $b \lesssim$ $-1.4^{\circ}$. This was indirectly noted by Glass et al., who noticed that the average period of the stars in this field at $b=-0.05^{\circ}$ is $427 \mathrm{~d}$ (and that of the known OH/IR stars $524 \mathrm{~d}$ ), while the average period in the Sgr I window $\left(b=-2.6^{\circ}\right)$ is $333 \mathrm{~d}$, with no known OH/IR stars (GWCF).

To quantify the nature of the Mira Bulge population, synthetic AGB evolutionary models were calculated, which are described in detail in Appendix C.

In brief, the synthetic AGB code of Wagenhuber \& Groenewegen (1998) was fine-tuned to reproduce the models of Vassiliadis \& Wood (1993) and then extended to more initial masses, including mass loss on the RGB. For several initial masses the fundamental mode period distribution was calculated for stars inside the observed instability strip for which the mass loss was below a critical value to simulate the fact that they should be optically visible. Vassiliadis \& Wood (1993) provide calculations for 4 different metal abundances: $Z=0.016,0.008,0.004$, and 0.001 . We used the models for $Z=0.016$, representing a solar mix, which is most appropriate for our Bulge sample (e.g. Rich 1998). We also show that our results are essentially unchanged if $Z=0.01$ or 0.02 are adopted. From the comparison of the observed period distribution for fields more than $1.2^{\circ}$ away from the galactic centre with the theoretical ones, we deduced that the periods can be explained with a population of stars with Main Sequence masses in the range of 1.5 to $2.0 M_{\odot}$. A possible extension to smaller masses is possible, but not necessary to explain the periods below 200 days. To explain the excess periods in the range of 350-600 days observed closer to the centre, we need initial masses in the range $2.5-3 M_{\odot}$. The presence of more massive stars in the inner field at $b=-0.05^{\circ}$ cannot be excluded, as it turns out that for more massive stars, the optically visible Mira phase is essentially absent. Sevenster (1999) analyses OH/IR stars (which are LPVs with longer periods and higher mass loss rates than the Miras) in the inner Galaxy and come to the conclusion that $\mathrm{OH} / \mathrm{IR}$ stars in the bulge have a minimum intial mass of about $1.3 M_{\odot}$, based on an analysis of infrared colours, compatible with our results. We briefly mention here the result from Olivier et al. (2001) who studied a sample of LPVs in the solar neighbourhood with periods in the 300 to 800 days range. They conclude that a majority of these stars had initial masses in the range of $1-2 M_{\odot}$, with an average value of $1.3 M_{\odot}$, lower than what we find for the 300 to 600 day range sample. This difference may be explained by the fact that our conclusions are only valid for a sample with no, or only low, mass loss rates $\left(\lesssim 5 \times 10^{-6} M_{\odot} / \mathrm{yr}\right)$, contrary to their sample which was selected to contain stars with significant mass loss $\left(\sim 10^{-5} M_{\odot} / \mathrm{yr}\right)$. As can be seen in the Vassiliadis \& Wood (1993) models, the period increases considerably when the stellar mass is reduced by the mass loss process.

We did not see a variation in the period distributions for the higher latitude fields (beyond $1^{\circ}$ latitude) and can consider this as a homogeneous "bulge" population, which according to the Vassiliadis \& Wood (1993) model, has ages in the range of 1 to about 3 Gyr. The excess population closer to the Galactic Centre is younger than 1 Gyr. According to Launhardt et al. (2002), the Nuclear Bulge (approximately the central degree) contains, besides the bulge population seen at higher latitudes, an additional population due to recent star formation closer to 

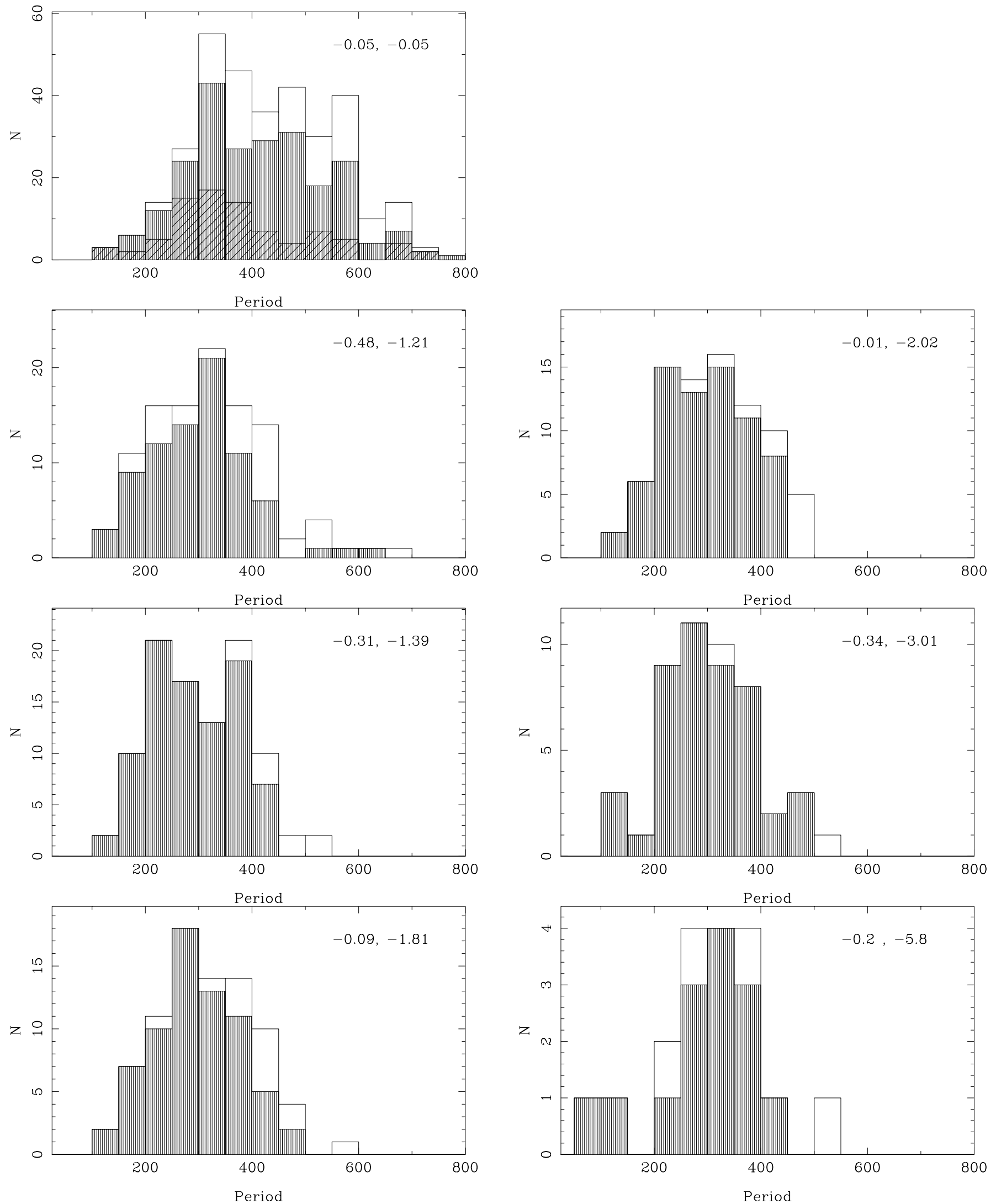

Fig. 6. Mira period distribution for 7 fields with similar longitudes but a range in latitudes (as indicated in the top right corner). For the field at $b \sim-5.8^{\circ}$, OGLE fields 6 and 7 have been combined. For the shaded histograms only stars with $(J-K)_{0}<2.0$ have been included. The field at $(-0.05,-0.05)$ is based on Glass et al. (2001); see main text for details. The histogram with slanted hatching is for the reddening by Schultheis et al. (1999b) for stars in this field, the shaded histogram for the adopted reddening which is 1.35 times larger. 


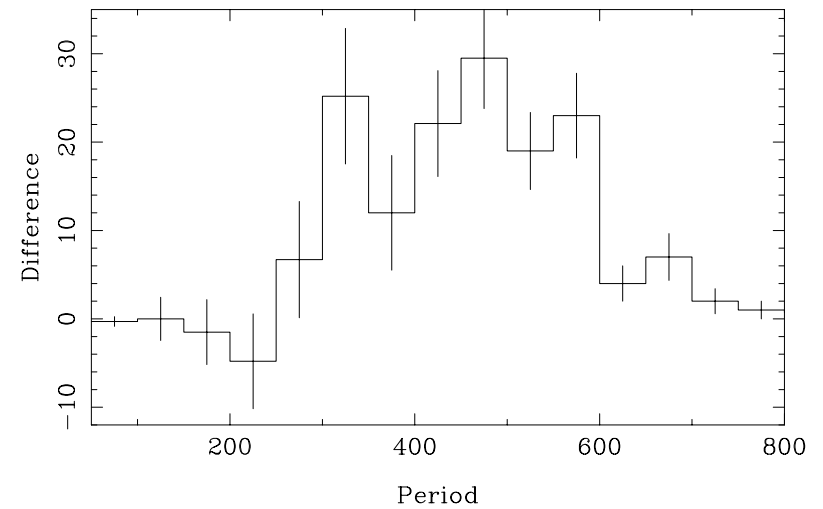

Fig. 7. Period distribution of the stars in the $b=-0.05^{\circ}$ field with $(J-K)_{0}<2.0$ minus the suitably scaled period distribution of the stars in the $b=-1.39$ to $-5.8^{\circ}$ fields with $(J-K)_{0}<2.0$. The scaling is such that below $\lesssim 300$ days the two distributions cancel at the $1 \sigma$ level.

the galactic centre. Blommaert et al. (1998) find that the extrapolation of the number density of bulge $\mathrm{OH} / \mathrm{IR}$ stars towards the galactic centre would explain half of the galactic centre $\mathrm{OH} / \mathrm{IR}$ population, but that an additional population, intrinsic to the galactic centre, exists, which agrees with what we see in the distribution here.

The formation history of the Bulge is still a matter of debate. In several works such as Kuijken \& Rich (2002) and recently in Zoccali et al. (2004), the bulge is considered to be old $(>10 \mathrm{Gyr})$ and formed on a relatively short timescale $(<1 \mathrm{Gyr})$ (e.g. Ferreras et al. 2003). On the basis of the modelling of colour-magnitude diagrams, Zoccali et al. claim that no trace has been found of any younger stellar population than $10 \mathrm{Gyr}$. The bulge Miras do not fit in this picture as they are considerably younger, according to our analysis. The field studied by Zoccali et al. is centred at $(l, b)=(0.277,-6.167)$ so at a slighter higher latitude than our extreme fields $\left(b \approx-5.8^{\circ}\right)$. Although we were limited by the small number of Miras detected at the highest latitude fields, we did not see a change in period distribution for those fields. Zoccali et al. (2004) acknowledge the presence of Miras, but consider them as part of the old population. It is true that Miras are also detected in globular clusters and thus can be associated with old ages, as is the case for a $1 M_{\odot}$ star in the Vassiliadis \& Wood (1993) model, but these stars produce periods shorter than 200 days (Fig. C.1), insufficient to explain the period distribution seen in the bulge. The periods of Miras in Globular Clusters range from 150 to 300 days (Frogel \& Whitelock 1998) and so only overlap with the shorter periods of the bulge Miras.

Our results agree more with the analysis of the infrared ISOGAL survey discussed in van Loon et al. (2003). They conclude that the bulk of the bulge population is old (more than 7 Gyr) but that a fraction of the stars is of intermediate age ( 1 to several Gyr). The Miras in our study can thus be considered as the intermediate age population seen in their analysis. van Loon et al. also see evidence of an even younger population $(<200 \mathrm{Myr})$, but according to our findings, this would be restricted to the area close to the Galactic Centre.

Our discussion of the ages of the Mira stars is based on the assumption that they have evolved from single stars.
An alternative scenario suggested by Renzini \& Greggio (1990) would be that the brighter (longer period) Miras could evolve from close binaries where the components coalesced to form one single star. This could lead to an underestimation of the age as the Mira essentially is the product of lower mass and thus older stars. This scenario may seem in better agreement with the idea that the bulge consists of an old stellar population. It does however, suffer from the same problem as the intermediate age population, in the sense that no clear evidence for Blue Stragglers (which would be the Main Sequence counterpart of the Miras) is found (Kuijken \& Rich 2002).

If indeed the bulk of the bulge population is old and formed quickly and if the Miras are of intermediate age, then our Miras must be representatives of a population which was added at a later stage, and it is unclear how it relates to the overall bulge. An interesting scenario suggested in Kormendy \& Kennicutt (2004) is the one in which a secondary bulge, also called pseudo-bulge, forms within an old bulge. Such a process would be connected to the presence of a "bar" which would add "disky" material into the old classical bulge. The Miras are indeed situated in a bar-structure, as discussed in the following section.

\section{The orientation of the bar}

Table 6 lists the zero points (ZPs) of the the $K$-band $P L$-relation (for a fixed slope of -3.37 ) for the individual OGLE fields. To increase the statistics, some neighbouring fields have been added together, as indicated in the first column of the table. The galactic coordinates listed are the mean values of all individual objects, rather than the mean of the field centres. Figure 9 plots these ZPs (with error bars) as a function of Galactic longitude. There is a clear correlation as the formal weighted fit has a slope of $-0.023 \pm 0.005$ (magnitude/degree). Restricting the fields to those with longitudes $-5<l<+5$ (reducing the contamination by disk stars, see Appendix B) the fit becomes:

$m_{\mathrm{K}}=(-0.0192 \pm 0.0087) l+(15.484 \pm 0.019)$

with an rms of 0.10 and based on 32 fields.

The interpretation of this correlation is that the Bulge Miras are located in the Galactic Bar that has a certain orientation towards the observer. A similar correlation was found by Wray (2004), who concluded that an appropriately chosen ZP in $I$ for the small amplitude OGLE variables in their sample (which they identify as corresponding to in Box "A-") correlated with Galactic longitude. No estimate for the orientation of the Bar was given however.

In Appendix B Monte Carlo simulations were carried out in order to quantify two issues: can these observations be used to constrain the orientation of the Galactic Bar, and, second, given the specific location of the OGLE fields, if there is any bias in the derived zero point compared to a fiducial $\mathrm{ZP}$, when all Miras would be located exactly in the Galactic Centre (GC). As described in Appendix B, for a spatial distribution of Bulge and Disk stars following Binney et al. (1997), viewing angles $\phi$ of 43 and 79 degrees (see the orientation in Fig. 8) result in slopes (magnitude versus $l$, Eq. (2)) in agreement with observations. However, the model with $\phi=43^{\circ}$ gives a much better fit to 
Table 6. Zero point of the $K$-band $P L$-relation.

\begin{tabular}{|c|c|c|c|c|}
\hline Fields & $\begin{array}{r}l^{(a)} \\
(\mathrm{deg})\end{array}$ & $\begin{array}{r}b^{(a)} \\
(\mathrm{deg})\end{array}$ & $\begin{array}{r}\mathrm{ZP}^{(b)} \\
(\mathrm{mag})\end{array}$ & $\mathbf{N}^{(c)}$ \\
\hline $8,9,10,11$ & 9.98 & -3.75 & $15.58 \pm 0.59$ & 23 \\
\hline 12,13 & 7.74 & -3.50 & $15.43 \pm 0.44$ & 27 \\
\hline 15 & 5.34 & +2.59 & $15.38 \pm 0.48$ & 22 \\
\hline 17 & 5.21 & -3.50 & $15.36 \pm 0.47$ & 24 \\
\hline 14 & 5.14 & +2.73 & $15.36 \pm 0.38$ & 29 \\
\hline 16 & 5.04 & -3.37 & $15.41 \pm 0.62$ & 17 \\
\hline 42 & 4.29 & -3.52 & $15.46 \pm 0.45$ & 13 \\
\hline 19 & 3.98 & -3.41 & $15.35 \pm 0.28$ & 20 \\
\hline 18 & 3.90 & -3.19 & $15.37 \pm 0.45$ & 20 \\
\hline 35,36 & 3.04 & -3.18 & $15.42 \pm 0.39$ & 29 \\
\hline 33 & 2.24 & -3.72 & $15.55 \pm 0.37$ & 22 \\
\hline 32 & 2.26 & -3.22 & $15.57 \pm 0.37$ & 24 \\
\hline 31 & 2.15 & -3.01 & $15.52 \pm 0.41$ & 32 \\
\hline 2 & 2.08 & -3.59 & $15.49 \pm 0.39$ & 19 \\
\hline 30 & 1.90 & -2.90 & $15.56 \pm 0.41$ & 25 \\
\hline 21 & 1.77 & -2.70 & $15.50 \pm 0.44$ & 33 \\
\hline 20 & 1.64 & -2.52 & $15.42 \pm 0.43$ & 41 \\
\hline 34 & 1.33 & -2.43 & $15.29 \pm 0.33$ & 35 \\
\hline 46 & 1.08 & -4.14 & $15.46 \pm 0.32$ & 18 \\
\hline 1 & 0.94 & -3.69 & $15.51 \pm 0.34$ & 22 \\
\hline 45 & 1.00 & -3.96 & $15.44 \pm 0.51$ & 15 \\
\hline 38 & 0.88 & -3.49 & $15.44 \pm 0.36$ & 25 \\
\hline 39 & 0.48 & -2.26 & $15.30 \pm 0.39$ & 69 \\
\hline 4 & 0.30 & -2.10 & $15.48 \pm 0.46$ & 54 \\
\hline 43 & 0.21 & +2.84 & $15.42 \pm 0.35$ & 47 \\
\hline 3 & -0.01 & -2.02 & $15.43 \pm 0.40$ & 70 \\
\hline 37 & -0.09 & -1.81 & $15.34 \pm 0.38$ & 68 \\
\hline 7 & -0.09 & -5.86 & $15.67 \pm 0.36$ & 6 \\
\hline 5 & -0.31 & -1.39 & $15.38 \pm 0.42$ & 90 \\
\hline 6 & -0.28 & -5.70 & $15.80 \pm 0.46$ & 8 \\
\hline 22 & -0.34 & -3.01 & $15.50 \pm 0.42$ & 46 \\
\hline 44 & -0.48 & -1.26 & $15.36 \pm 0.36$ & 79 \\
\hline 23 & -0.63 & -3.44 & $15.53 \pm 0.40$ & 30 \\
\hline 25 & -2.41 & -3.64 & $15.44 \pm 0.31$ & 33 \\
\hline 24 & -2.56 & -3.46 & $15.62 \pm 0.34$ & 29 \\
\hline 41 & -2.86 & -3.35 & $15.63 \pm 0.38$ & 24 \\
\hline 40 & -3.16 & -3.27 & $15.50 \pm 0.37$ & 34 \\
\hline 26,27 & -4.99 & -3.58 & $15.63 \pm 0.42$ & 39 \\
\hline 28,29 & -6.75 & -4.55 & $15.55 \pm 0.42$ & 13 \\
\hline $47,48,49$ & -11.19 & -2.78 & $15.95 \pm 0.35$ & 18 \\
\hline
\end{tabular}

${ }^{(a)}$ Mean values of individual objects. ${ }^{(b)}$ Zero point of the $K$-band $P L$ relation for a slope of -3.37 . ${ }^{(c)}$ Number of objects in Box " $C$ " used to calculate the $P L$-relation.

the number of stars per field. The bias in the ZPs is essentially independent of viewing angles, and for the best fitting model the observed ZP derived for all stars (Eq. (1)) is too bright by $0.018 \mathrm{mag}( \pm 0.013)$, while the ZP in Eq. (2) is too bright by $0.002( \pm 0.021)$ mag.

The preferred value of $\phi=43^{\circ}$ is in agreement with the values of about $45^{\circ}$ by Whitelock (1992), based on 104 IRAS detected Mira variables, and the preferred value of $46^{\circ}$ by Sevenster et al. (1999), based on an analysis of OH/IR stars in the inner Galaxy.

Other values in the literature are usually much larger, between 60 and 80 degrees: Dwek et al. (1995) and

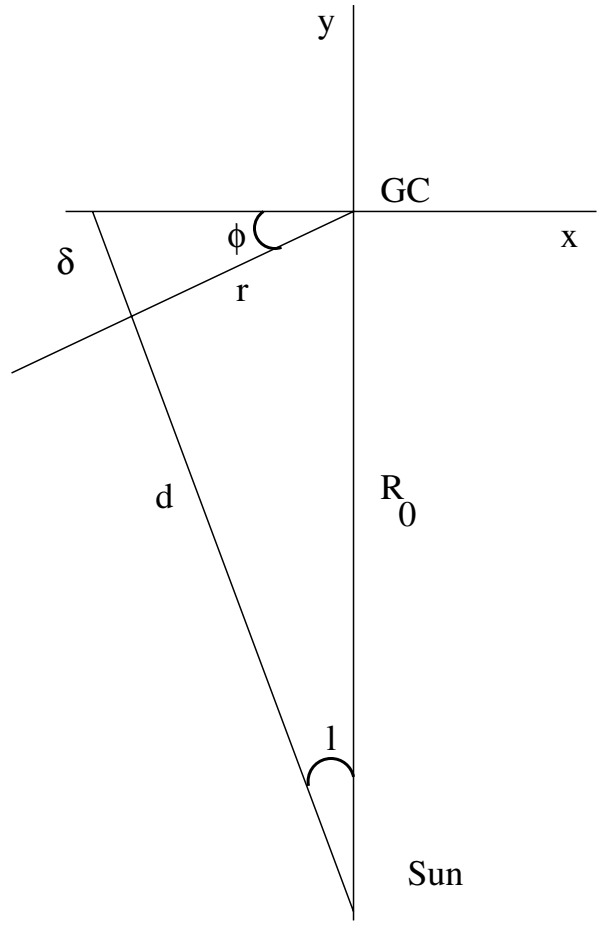

Fig. 8. Schematic drawing of the orientation of the major-axis of the Galactic Bar w.r.t. the Sun and the Galactic Centre. The $z$-axis is directed towards the reader. See Appendix B.
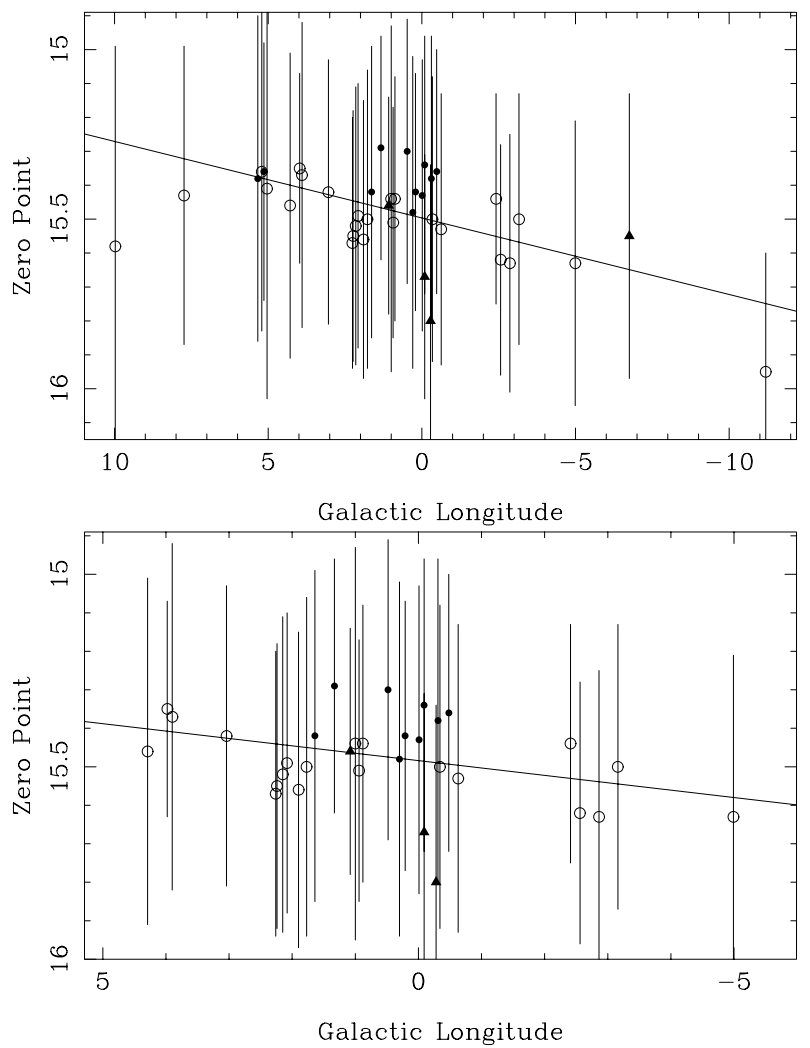

Fig. 9. Zero point of the $K$-band $P L$-relation as a function of Galactic longitude. Galactic latitudes below -4.0 are indicated by filled triangles, those larger than -2.6 by filled circles, and the remaining by open circles. Error bars are also plotted. The lines represent linear leastsquares fits to all fields (top panel), and those with $|l|<5^{\circ}$ (bottom panel). 
Binney et al. (1997), based on COBE-DIRBE data; Stanek et al. (1997), based on bulge red clump stars; Robin et al. (2003) and Picaud \& Robin, based on colour-magnitude fitting. Sevenster et al. (1999), however, argue that these values are commonly found when no velocity data is available, the longitude range is too narrow, or when low latitudes are excluded. It is also possible that these studies are tracing other populations, which may be distributed differently than the Miras. Whitelock et al. and Sevenster et al. do use populations closely related to the Mira stars and find an angle of the bar close to the one we derive.

\section{The distance to the galactic centre}

A slope of the $K$-band $P L$-relation of $-3.37 \pm 0.09$ was derived, a quantity which has seldom been determined before. GS03 derived a $P L$-relation in NGC 6522 based on 34 MACHO variables with $r$-amplitude $>1.5$ and DENIS $K$ photometry: $m_{\mathrm{K}}=-4.6 \log P+18.1$. No errors or rms were given, as by their own account - this fit was made by eye. Much better agreement is found with GWCF. Based on multi-epoch data of 55 stars they found $m_{\mathrm{K}}=(-3.47 \pm 0.35) \log P+(15.64 \pm 0.86)$ $(\mathrm{rms}=0.35)$ in the Sgr I field.

Zero points for the $K$-band $P L$-relation were derived in two ways. First, a direct fit to all stars resulting in (15.44 \pm 0.21$)$, and secondly, determining ZPs per (sub)-field, and fitting this as a function of $l$, resulting in $(15.484 \pm 0.019)$. Applying the small bias corrections discussed at the end of Sect. 9 and averaging over the two estimates, the adopted $K$-band $P L$-relation for Miras at the GC is:

$m_{\mathrm{K}}=-3.37 \log P+(15.47 \pm 0.03)$.

The derived $P L$-relation can be compared to the one derived for 83 O-rich LPVs in the LMC derived in G04: $m_{\mathrm{K}}=(-3.52 \pm$ $0.16) \log P+(19.56 \pm 0.38)$, with an rms of 0.26 . Since the slopes are not exactly the same, the magnitudes were compared at the approximate mean period of $\log P=2.45$. The difference in magnitude is 3.72. Adopting the LMC based slope of -3.52 for the GB Miras and re-fitting the ZP, the bias corrected ZP would become 15.85 , resulting in a GB-LMC DM difference of 3.71, essentially the same value. If the distance to the GC is assumed to be $7.94 \mathrm{kpc}$ (Eisenhauer et al. 2003; in a recent preprint this was even lowered to $7.62 \pm 0.32 \mathrm{kpc}$, Eisenhauer et al. 2005), then the LMC would be at a DM $=18.21$; or if the DM to the LMC is assumed to be 18.50 (Walker 2003; Feast 2004a), then the GC would be at $9.0 \mathrm{kpc}$. A similar result was found by GWCF, who derived a distance to the GC of $8.9 \pm 0.7 \mathrm{kpc}$, assuming 18.55 for the LMC DM and $\phi=45^{\circ}$. The analysis so far has assumed no metallicity dependence of the Mira $P L$-relation. Wood (1990) present linear non-adiabatic pulsation calculations that suggest a dependence of the form $\log P \sim 0.46 \log Z+1.59 \log L$, but he notes that in the $K$ band the dependence is expected to be weaker, and following the example he presents, one infers a dependence of $0.25 \log Z$ in the $K$-band. In G04, $K$-band $P L$-relations were derived for carbon-miras in the SMC and LMC. At a characteristic period of $\log P=2.45$, one infers a relative difference in DM of 0.38 , which is smaller than the commonly quoted value of near $0.50(0.48-0.53 \pm 0.11$, FO cepheids, Bono et al. 2002;
$0.46-0.51 \pm 0.15$, FU cepheids, Groenewegen $2000 ; 0.44 \pm 0.05$, TRGB, Cioni et al. 2000). This may hint at a metallicity dependence of the Mira $K$-band $P L$-relation. To test this hypothesis, a correction to the $K$-magnitude of $+\beta \log Z$ was assumed ${ }^{3}$ (for both O- and C-rich LPVs), and the Bulge, LMC, and SMC was assumed to have solar, solar $/ 2$, and solar/4 metallicity, respectively. For a value $\beta=0.25$ the relative SMC-LMC DM based on the C-Miras was increased from 0.38 to 0.46 , while the relative DM LMC-GC was increased from 3.72 to 3.80 . If the relative SMC-LMC DM is fixed at 0.50 , then $\beta=0.40$ is required, and the relative DM LMC-GC becomes 3.84 for that value. For an LMC DM of 18.50, the distance to the GC then becomes $8.6 \mathrm{kpc}$. The error in this value is somewhat difficult to estimate, as the $P L$-relations derived in G04 and here are from - at best - the average of two $K$ values. Work by Feast et al. (1989) indicates that in the case of multi-epoch data (and for the small depth effect in the LMC), the intrinsic dispersion in the $P L$-relation is about $0.13 \mathrm{mag}$. Therefore we assign an error of 0.18 to the difference in DM, which implies an error of $0.7 \mathrm{kpc}$. Based on this large sample of Mira variables in the direction of the $\mathrm{GB}$, the conclusion is that the distance to the $\mathrm{GC}$ is between 8.6 and $9.0( \pm 0.7) \mathrm{kpc}$, depending on the metallicity dependence of the $K$-band $P L$-relation. Feast (2004b) discusses the zeropoint of the Mira $K$-band $P L$-relation and, adopting the slope observed in the LMC (-3.47), derives a zeropoint of $1.00 \pm 0.08$, averaging over independently derived ZPs from trigonometric parallaxes, OH VLBI expansion parallaxes and Galactic Globular Clusters. Adopting a slope of -3.47 and refitting the $\mathrm{ZP}$ of the Bulge sample, the bias corrected value becomes $15.73 \pm 0.03$ and, without metallicity correction (consistent with the assumption above about the metallicities in Bulge, LMC, SMC), leads to a distance to the GC of $8.8 \pm 0.4 \mathrm{kpc}$. This independent distance estimate is in between the values derived using no or a strong metallicity dependent zero point.

Acknowledgements. This research made use of the SIMBAD database, operated at the CDS, Strasbourg, France. This publication makes use of data products from the Two Micron All Sky Survey, which is a joint project of the University of Massachusetts and the Infrared Processing and Analysis Center/California Institute of Technology, funded by the National Aeronautics and Space Administration and the National Science Foundation.

\section{References}

Alard, C., Terzan, A., \& Guibert, J. 1996, A\&AS, 120, 275

Alard, C., Blommaert, J. A. D. L., Cesarsky, C., et al. 2001, ApJ, 552, 289 (ABC01)

Binney, J., Gerhard, O., \& Spergel, D. 1997, MNRAS, 288, 365

Blanco, B. M. 1984, AJ, 89, 1836 (B84)

Blanco, B. M. 1986, AJ, 91, 290

Blanco, V. M., McCarthy, M. F., \& Blanco, B. M. 1984, AJ, 89, 636 (BMB)

Blommaert, J. A. D. L., van der Veen, W. E. C. J., van Langevelde, H. J., Habing, H. J., \& Sjouwerman, L. O. 1998, A\&A, 329, 991

\footnotetext{
${ }^{3}$ That is, $M_{\mathrm{K}}=M_{\mathrm{K}}($ ref $)+\beta \log \left(Z / Z_{\text {ref }}\right)$, where $M_{\mathrm{K}}($ ref $)$ is the known magnitude in a galaxy with metallicity $Z_{\mathrm{ref}}$, and $M_{\mathrm{K}}$ the magnitude it would have in a galaxy with metallicity $Z$.
} 
Bono, G., Groenewegen, M. A. T., Marconi, M., \& Caputo, F. 2002, ApJ, 574, L33

Catchpole, R. M. 1982, MNRAS, 200, 33p

Cieslinski, D. 2003, PASP, 115, 193

Cioni, M.-R. L., Blommaert, J. A. D. L., Groenewegen, M. A. T., et al. 2003, A\&A, 406, 51

Cioni, M.-R. L., van der Marel, R. P., Loup, C., \& Habing, H. J. 2000, A\&A, 359, 601

Cutri, R. M., Skrutskie, M. F., Van Dyk, S., et al. 2003, Explanatory Supplement to the 2MASS All-Sky Data Release

DENIS consortium 2003, http://vizier.u-strasbg.fr/ viz-bin/VizieR?-source=DENIS2

Dominici, T. P., Horvath, J. E., Medina Tanco, G. A., Teixeira, R., \& Benevides-Soares, P. 1999, A\&AS, 139, 321 (DHM99)

Draine, B. T. 2003, ARA\&A, 41, 241

Dutra, C. M., \& Bica, E. 2000, A \& A 359, L9

Dwek, E., Arendi, R. G., Hauser, M. G., et al. 1995, ApJ, 445, 716

Eisenhauer, F., Schödel, R., Genzel, R., et al. 2003, ApJ, 597, L12

Eisenhauer, F., Genzel, R., Alexander, T., et al. 2005, ApJ, 628, 24

Epchtein, N., Deul, E., Derriere, S., et al. 1999, A\&A, 349, 236

Feast, M. W. 2004a, in Nearby Large-Scale Structures and the Zone of Avoidance, ed. A. P. Fairall, \& P. Woudt, ASP Conf. Ser, in press [arXiv:astro-ph/0405440]

Feast, M. W. 2004b, in Variable Stars in the Local Group, ed. D. W. Kurtz, \& Karen Pollard, IAU Coll., 193, ASP Conf. Ser., 310, 304

Feast, M. W., Glass, I. S., Whitelock, P. A., \& Catchpole, R. M. 1989, MNRAS, 241, 375

Felli, M., Testi, L., Schuller, F., \& Omont, A. 2002, A\&A, 392, 971

Ferreras, I., Wyse, R. F. G., \& Silk, J. 2003, MNRAS, 355, 64

Fluks, M. A., Plez, B., Thé, P. S., et al. 1994, A\&AS, 105, 311

Fraser, O. J., Hawley, S. L., Cook, K. H., \& Keller, S. C. 2005, AJ, 129,768

Frogel, J. A., \& Whitelock, P. A. 1998, AJ, 116, 754

Frogel, J. A., \& Whitford, A. E. 1987, ApJ, 320, 199 (FW87)

Glass, I. S. 1986, MNRAS, 221, 879 (G86)

Glass, I. S., \& Feast, M. W. 1982, MNRAS, 198, 199 (GF82)

Glass, I. S., \& Schultheis, M. 2002, MNRAS, 337, 519 (GS02)

Glass, I. S., \& Schultheis, M. 2003, MNRAS, 345, 39 (GS03)

Glass, I. S., Whitelock, P. A., Catchpole, R. M., \& Feast, M. W. 1995, MNRAS, 273, 383 (GWCF)

Glass, I. S., Ganesh, S., Alard, C., et al. 1999, MNRAS, 308, 127

Glass, I. S., Matsumoto, S., Carter, B. S., \& Sekiguchi, K. 2001, MNRAS, 321, 77; erratum: 2002, MNRAS, 336, 1390

Groenewegen, M. A. T. 1993, Ph.D. Thesis, Chap. 5, University of Amsterdam

Groenewegen, M. A. T. 2000, A\&A, 363, 901

Groenewegen, M. A. T. 2004, A\&A, 425, 595 (G04)

Hughes, S. M. G. 1989, AJ, 97, 1634

Ita, Y., Tanabé, T., Matsunaga, N., et al. 2004, MNRAS, 347, 720

Izumiura, H., Deguchi, S., Hashimoto, O., et al. 1995, ApJ, 453, 837

Kiss, L. L., \& Bedding, T. 2003, MNRAS, 343, L79

Kiss, L. L., \& Bedding, T. 2004, MNRAS, 347, L83

Kormendy, J., \& Kennicutt, C., Jr. 2004, ARAA, 42, 603

Kohoutek, L. 2002, AN 323, 57

Kuijken, K., \& Rich, R. M. 2002, AJ, 124, 2054

Kwok, S., Volk, K., \& Bidelman, W. P. 1997, ApJS, 112, 557

Lançon, A., \& Wood, P. 2000, A\&AS, 146, 217

Launhardt, R., Zylka, R., \& Mezger, P. G. 2002, A\&A, 384, 112

Lebzelter, T., Schulteis, M., \& Melchior, A. L. 2002, A\&A, 393, 573

Lloyd-Evans, T. 1976, MNRAS, 174, 169 (LE76)

Maíz-Apellániz, J. 2001, AJ, 121, 2737

McWilliam, A., \& Rich, R. R. 1994, ApJS, 91, 749

Medina Tanco, G. A., \& Steiner, J. E. 1995, AJ, 109, 1770
Messineo, M., Habing, H. J., Sjouwerman, L. O., Omont, A., \& Menten, K. M. 2002, A\&A, 393, 115

Munari, U., \& Zwitter, T. 2002, A\&A, 383, 188

Noda, S., Takeuti, M., Abe, F., et al. 2002, MNRAS, 330, 137

Ojha, D. K., Omont, A., Schuller, F., et al. 2003, A\&A, 403, 141 (OOS03)

Olivier, E. A., \& Wood, P. R. 2003, ApJ, 584, 1035

Omont, A., Ganesh, S., Alard, C., et al. 1999, A\&A, 348, 755

Paunzen, E., Maitzen, H. M., Rakos, K. D., \& Sschombert, J. 2003, A\&A, 403, 937

Picaud, S., \& Robin, A. C. 2004, A\&A, 428, 891

Popowski, P., Cook, K. H., \& Becker, A. C. 2003, AJ, 126, 2910

Raharto, M., Hamajima, K., Ichikawa, T., Ishida, K., \& Hidayat, B. 1984, AnTok 19, 46

Renzini, A., \& Greggio, L. 1990, in Bulges of Galaxies, ESO-CTIO workshop No. 35, ed. B. J. Jarvis, \& D. M. Terndrup, 47

Rich, R. M. 1998, in The Central Regions of the Galaxy and Galaxies (Dordrecht: Kluwer), ed. Sofue Y., IAU Symp., 184, 11

Robin, A. C., Reylé, C., Derrière, \& Picaud, S. 2003, A\&A, 409, 523; erratum: 2004, A\&A, 416, 157

Sevenster, M. N. 1999, MNRAS, 310, 629

Sevenster, M. N., Chapman, J. M., Habing, H. J., Killeen, N. E. B., \& Lindqvist, M. 1997, A\&AS, 122, 79

Sevenster, M. N., Saha, P., Valls-Gabaud, D., \& Fux, R. 1999, MNRAS, 307, 584

Schultheis, M., \& Glass, I. S. 2001, MNRAS, 327, 1193

Schultheis, M., Aringer, B., Jorgensen, U. G., Lebzelter, T., \& Plez, B. 1999a, in Abstract of the 2nd Austrian ISO workshop Atmospheres of M, S and C giants, Vienna, Austria, ed. J. Hron, \& S. Höfner, 93

Schultheis, M., Ganesh, S., Simin, G., et al. 1999b, A\&A, 349, L69

Sivagnnanam, P., Braz, M. A., Le Squeren, A. M., \& Tran Minh, F. 1990, A\&A, 233, 112

Stanek, K. Z., Udalski, A., Szymański, M., et al. 1997, ApJ, 477, 163

Sumi, T. 2004, MNRAS, 349, 193

Sumi, T., Wu, X., Udalski, A., et al. 2004, MNRAS, 348, 1439

te Lintel Hekkert, P., Caswell, J. L., Habing, H. J., Haynes, R. F., \& Norris, R. P. 1991, A\&AS, 90, 327

Terzan, A., \& Gosset, E. 1991, A\&AS, 90, 451 (TG91)

Terzan, A., \& Ounnas, C. 1988, A\&AS, 76, 205 (TO88)

Udalski, A., Kubiak, M., Szymański, M., et al. 1994, AcA, 44, 317

Udalski, A., Szymański, M., Kaluzny, J., et al. 1995a, AcA, 45, 1

Udalski, A., Olech, A., Szymański, M., et al. 1995b, AcA, 45, 433

Udalski, A., Olech, A., Szymański, M., et al. 1996, AcA, 46, 51

Udalski, A., Olech, A., Szymański, M., et al. 1997, AcA, 47, 1

Udalski, A., Szymański, M., Kubiak, M. et al. 2002, AcA, 52, 217

Vassiliadis, E., \& Wood, P. R. 1993, ApJ, 413, 641

Wagenhuber, J., \& Groenewegen, M. A. T. 1998, A\&A, 340, 183

Walker, A. R. 2003, in Stellar candles for the extragalactic distance scale, Lect. Notes Phys., 635, 265

Whitelock, P. A. 1992, in Variable stars and galaxies, ASPC Ser., 30, 11

Whitelock, P. A., Feast, M. W., \& Catchpole, R. M. 1991, MNRAS, 248, 276

Wood, P. R. 1990, in From Miras to Planetary Nebulae, ed. M. O. Mennessier, \& A. Omont (Gif-sur-Yvette: Éditions Frontières), 67

Wood, P. R. 2000, PASA 17, 18

Wood, P. R., Alcock, C., Allsman, R. A., et al. 1999, in AGB stars, ed. T. Le Bertre, A. Lèbre, \& C. Waelkens (Kluwer Academic Publishers, ASP), IAU Symp., 191, 151

Wood, P. R., Olivier, E. A., \& Kawaler, S. D. 2004, ApJ, 604, 800

Wozniak, P. R., Udalski, A., Szymanski, M., et al. 2002 AcA, 52, 129

Wray, J. J., Eyer, L., \& Paczynski, B. 2004, MNRAS, 349, 1059

Zoccali, M., Renzini, A., Ortolani, S., et al. 2003, A\&A, 399, 931 
M. A. T. Groenewegen and J. A. D. L. Blommaert: Mira variables in the bulge, Online Material $p 1$

\section{Online Material}


M. A. T. Groenewegen and J. A. D. L. Blommaert: Mira variables in the bulge, Online Material p 2

\section{Appendix A: The light curve analysis model}

Some small changes w.r.t. the implementation of the lightcurve analysise model in G04 are described.

The only change in the first part of the code is the level at which a period is accepted as significant. This level was set at significance $=1.0 \times 10^{-16}$, compared to $5.5 \times 10^{-11}$ in G04. This was possible as - contrary to G04 - only objects with large amplitudes were searched for. The resulting increase in the number of spurious periods was then caught in the process of visual inspection.

As in G04 a list of known objects (both known non-LPVs, and known AGB giants and LPVs) was compiled to ease automatic association. The list comprises:

(1) 14833 IRAS sources within $10 \mathrm{deg}$. radius of the centre of the 49 OGLE fields at RA $=268.87$, Dec $=-31.03^{4}$;

(2) 51141 ISOGAL sources from Omont et al. (2003, hereafter OGA03; those within the extreme values of the OGLE field boundaries $261.612<=$ RA $<=276.133$, $-40.726<=$ Dec $<=-21.328)$;

(3) 268 pulsating variable stars, 1650 Eclipsing Binaries, and 943 Miscellaneous variable stars from OGLE-I (Udalski et al. 1994, 1995a,b, 1996, 1997);

(4) 332 objects from Alard et al. (2001, hereafter ABC01), who correlated ISOGAL sources within the NGC 6522 and Sgr I Baade windows with the MACHO database;

(5) 2353 objects from Ojha et al. (2003, hereafter OOS03), who studied sources in 9 ISOGAL fields;

(6) 174 M-giants later than spectral type M0 in the NGC 6522 Baade's window from Glass \& Schultheis (2002, hereafter GS02);

(7) 421 objects from Glass et al. (2001, and erratum), who monitored in $K$ over four years an $24 \times 24$ arcmin area near the Galactic Centre;

(8) 122 objects from Alard et al. (1996, identified as Ter[number]), who identified LPV using red photographic plates;

(9) 494 objects from Lloyd-Evans (1976, hereafter LloydEvans (1976), who identified Mira variables in the three Baade windows, identified as TLE-[field]-[number] in Table 2), Blanco et al. (1984, hereafter BMB, who identified M giants in Baade's window), and Blanco (1984, hereafter B84, who identified RR Lyrae variables) with coordinates listed in the SIMBAD database to $1^{\prime \prime}$ or better;

(10) 33 Nova related objects from Cieslinski (2003). The total number of sources used in the automatic correlation is 72764 .

\section{Appendix B: Simulations of the Galactic bulge and foreground disk stars}

This appendix describes the calculations to model a population in the direction of the Galactic Bulge.

The basic model is essentially the one proposed by Binney et al. (1997) to model the dust-corrected near-infrared

\footnotetext{
${ }^{4}$ Retrieved from the infrared science archive at IPAC, http://irsa.ipac.caltech.edu/
}

COBE/DIRBE surface brightness map of the inner galaxy. The number density of bulge stars is assumed to be:

$f_{\mathrm{b}}=f_{0} \exp \left(-a^{2} / a_{\mathrm{m}}^{2}\right) /\left(1+a / a_{0}\right)^{\beta}$

with $f_{0}=624, a_{\mathrm{m}}=1.9 \mathrm{kpc}, a_{0}=0.10 \mathrm{kpc}, \beta=1.8$. Binney et al. assume a tri-axial bulge with axial ratios 1:0.6:0.4. For numerical convenience a prolate ellipsoid is assumed here: $a=\sqrt{x^{2}+(y / \eta)^{2}+(z / \eta)^{2}}$ with the value of $\eta=0.5$ taken from Binney et al.

The number of Bulge objects up to a radius $r$ from the centre, which defines the probability density function in the simulation, is approximated as

$N_{\mathrm{b}}(r)=\int_{0}^{r} 4 \pi a^{2} f_{\mathrm{b}}(a) \mathrm{d} a$

up to a maximum radius that is taken to be the co-rotation radius, with a default value of $R_{\mathrm{cr}}=2.4 \mathrm{kpc}$, following Dwek et al. (1995).

The number density of disk stars is assumed to be:

$$
\begin{aligned}
f_{\mathrm{d}}= & \left(\exp \left(-|z| / z_{0}\right)+\alpha \exp \left(-|z| / z_{1}\right)\right) \\
& \times R_{\mathrm{d}}\left(\exp \left(-r / R_{\mathrm{d}}\right)-f_{\mathrm{h}} \exp \left(-r / R_{\mathrm{h}}\right)\right)
\end{aligned}
$$

with $z_{0}=0.210 \mathrm{kpc}, z_{1}=0.042 \mathrm{kpc}, \alpha=0.27, R_{\mathrm{d}}=2.5 \mathrm{kpc}$ (Binney et al.) and $R_{\mathrm{h}}=1.3 \mathrm{kpc}$ (Picaud \& Robin 2004). This functional form follows Binney et al., but also allows for a "hole" in the inner disk (the scaling parameter is $0 \leq f_{\mathrm{h}} \leq 1$ and identical to zero in Binney et al.). The total number of disk stars and the probability density functions are defined as:

$N_{\mathrm{d}} \equiv N_{\mathrm{d}, z}(z) \times N_{\mathrm{d}, r}(r)$

given by,

$$
\begin{aligned}
N_{\mathrm{d}}= & {\left[2 \int_{0}^{z} \exp \left(-z / z_{0}\right)+\alpha \exp \left(-z / z_{1}\right) \mathrm{d} z\right] } \\
& \times\left[\int_{0}^{r} 2 \pi r R_{\mathrm{d}}\left(\exp \left(-r / R_{\mathrm{d}}\right)-f_{\mathrm{h}} \exp \left(-r / R_{\mathrm{h}}\right)\right) \mathrm{d} r\right]
\end{aligned}
$$

up to maximum values $z_{\max }=\left(R_{\mathrm{cr}} \eta\right)$, and $R_{\max }=8.0 \mathrm{kpc}$, respectively.

A disk or bulge star is generated according to the ratio $N_{\mathrm{b}} /\left(N_{\mathrm{b}}+N_{\mathrm{d}, z} \times N_{\mathrm{d}, r}\right)$. In the case of a disk star, its height above the plane $z$, distance to the GC $r$, and a random angle between 0 and $2 \pi$ in the Galactic plane are drawn according to the probability functions $N_{\mathrm{d}, z}(z)$ and $N_{\mathrm{d}, r}(r)$. Its coordinates $x, y, z$ are then known.

In the case of a bulge star, the distance $a$ to the GC is drawn according to the probability function $N_{\mathrm{b}}$, and then a star is randomly placed on the surface of the appropriate ellipsoid, to find $x, y, z$. These values are then rotated by an angle $\phi$ in the Galactic plane (see Fig. 8). The Galactic coordinates are then derived assuming a distance from the Sun to the GC of $R_{0}=8.5 \mathrm{kpc}$, and height above the plane of $z_{0}=+24 \mathrm{pc}$ (Maíz-Apellániz 2001)

In a second step the known distance to the Sun is used to calculate every stars appararent magnitude, assuming an arbitrary $M_{\mathrm{K}}$ of $-7.5 \mathrm{mag}$ with a Gaussian dispersion of $\sigma_{\mathrm{K}}=$ 0.15 mag. This is close to the dispersion observed in the 
$P L$-relation in LMC Miras when multi-epoch photometry is available to accurately determine mean-light magnitudes (Feast et al. 1989).

In a third step it is verified for every simulated star if it is located within one of the 40 lines-of-sight considered, listed in Table 6 . The field sizes of $14.2^{\prime} \times 57^{\prime}$ are approximated by a circle of radius 0.27 degrees. If so, it is assumed the star would have been "detected". Given the relative brightness of the LPVs it is assumed that completeness is not an issue.

The number of stars drawn was such that a total of about 1200 objects were "detected", similar to the actual number. At the end of the simulation, the average magnitude and dispersion per line-of-sight was determined, and a weighted least-square fit was made of the mean magnitude versus longitude for all fields and for those with $|l|<5^{\circ}$, as for the observations. In addition, the mean magnitude and dispersion for all "detected" stars was determined.

Such a simulation was repeated 1000 times. Then, distribution functions and from that median and 1-sigma values of the following parameters were determined: the number of stars (total, disk, bulge), mean magnitude and sigma (for every lineof-sight), mean magnitude and sigma for all stars, and slope and error in the slope both when fitted over all longitudes, and for those fields with $|l|<5^{\circ}$.

For the standard model of Binney et al. described above (i.e. $f_{\mathrm{h}}=0$ ), it turns out that for two values of $\phi$ a slope (because of the contamination by disk stars in the outer fields, the slope fitted over $|l|<5^{\circ}$ is considered from now on) in agreement with observations is found: $\phi=43$ and 79 degrees (with values between 25 and 85 degrees resulting in predicted slopes within 1- $\sigma$ of the observed one.)

Figures B.1-B.3 show the results for these two cases. Figure B.1 shows the distribution on the Galactic Plane for a random sub-sample of all stars simulated, and illustrates a fundamental difference between the two cases. For large viewing angles, the outer fields $|l| \gtrsim 10^{\circ}$ will be dominated by disk stars. Figure B.2 shows for the same random sub-sample the observed magnitude as a function of $l$. In Fig. B.3 the simulated mean magnitude and error for each field are compared to the observations in the top panel, while in the bottom panel the observed and predicted number of objects are compared. It is from this plot that one may conclude that the model with $\phi=43^{\circ}$ is to be preferred over the one with $\phi=79^{\circ}$ as the latter model predicts too few stars, especially in the outer fields, compared to the observations. Comparing only the observed and predicted number of stars (in an $\chi^{2}$ sense) a best fit is found for $\phi=35^{\circ}$ (with values between 0 and 60 degrees resulting in a reduced $\chi^{2}$ within 1 unit of the minimum $\chi^{2}$ ). Combining the constraints from the slope and the number of stars a viewing angle of $\phi=43 \pm 17$ degrees is the preferred value.

One may consider the ratio of Bulge to Disk stars as uncertain, and therefore, a model was considered with $\phi=79^{\circ}$ and $f_{0}=350$. The latter value was set so that the model predicted the observed number of stars in the $l=-11^{\circ}$ field. Such a model would still underestimate the number of stars in the outer fields at positive $l$, and would give a slope no longer in agreement with observations.
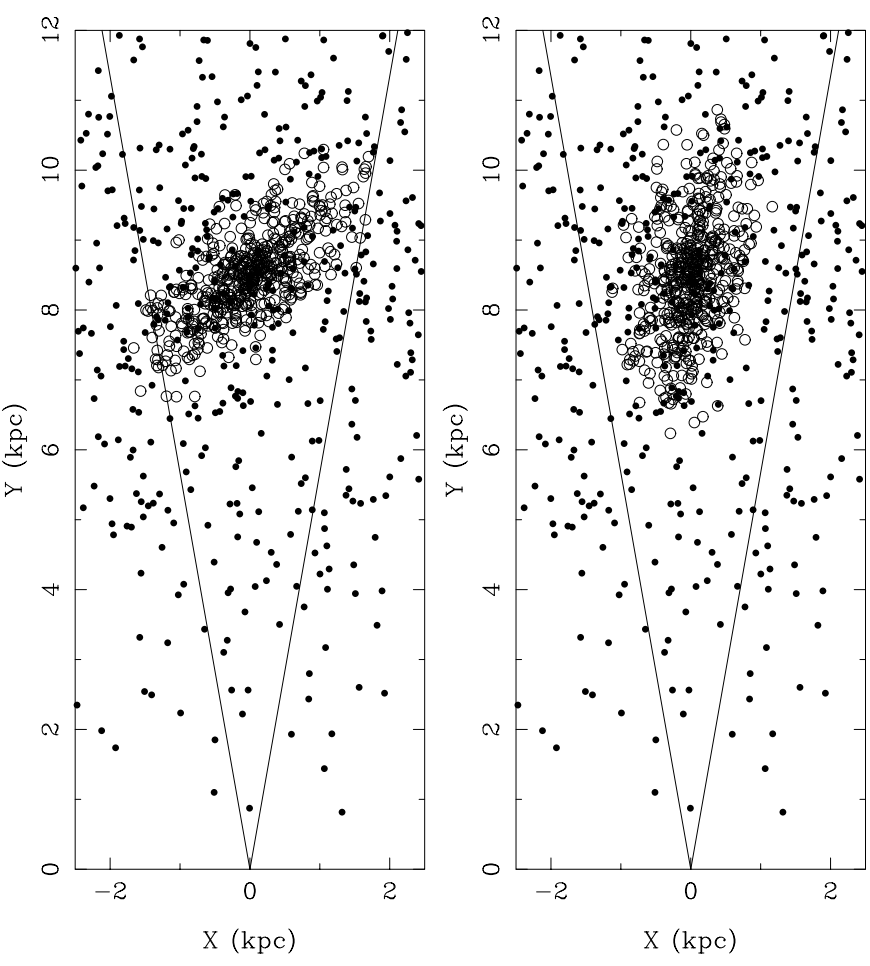

Fig. B.1. For angles of $\phi$ of 43 and 79 degrees (left and right panel), the projection (drawn to scale) on the Galactic Plane of 1300 randomly drawn stars. Disk stars are represented by filled circles, Bulge stars by open circles. The Sun is at $X=0, Y=0$. The Galactic Centre is at $X=$ $0, Y=8.5$. The lines illustrate Galactic longitudes of \pm 10 degrees.

Finally, a model including a hole in the inner disk was considered (i.e. $f_{\mathrm{h}}=1$ ). To have the same ratio of bulge to disk stars, $f_{0}$ was set to 425 . The results are very similar and the best fitting angle is now 40 degrees.

For reference, the predicted number of disk and bulge stars for the viewing angles of 43 and 79 degrees and for the model with $\phi=40^{\circ}$ and the central hole in the disk are listed in Table B.1. As they are quite different, these predictions may be useful when additional data (proper motion ${ }^{5}$, radial velocities) become available to constrain the ratio of disk to bulge objects as a function of galactic coordinates.

\section{Appendix C: Comparing stellar evolution codes}

The models described in Vassiliadis \& Wood (1993, VW) were downloaded from P. Wood's webpage (http://www.mso.anu.edu.au/ wood/). These files list the relevant stellar quantities (remaining stellar mass, luminosity, and effective temperature amongst other quantities), and the evolutionary time, for the individual calculated models on the AGB. They are available for $Z=0.016(1.0,1.5,2.0,2.5$, $\left.3.5,5.0 M_{\odot}\right), Z=0.008\left(0.945,1.0,1.5,2.0,2.5,3.5,5.0 M_{\odot}\right)$, $Z=0.004\left(0.89,1.0,1.5,2.0,2.5,3.5,5.0 M_{\odot}\right)$, and $Z=0.001$ $\left(1.0,1.5 M_{\odot}\right)$.

\footnotetext{
5 Sumi et al. (2004) present proper motions for 5 million objects in the OGLE fields, centred on the expected $I_{0}$ magnitude and $(V-I)_{0}$ colour of red clump giants but also including some red giants. Of the 2691 LPVs in the present study, 1612 are listed in Sumi et al.
} 
M. A. T. Groenewegen and J. A. D. L. Blommaert: Mira variables in the bulge, Online Material p 4
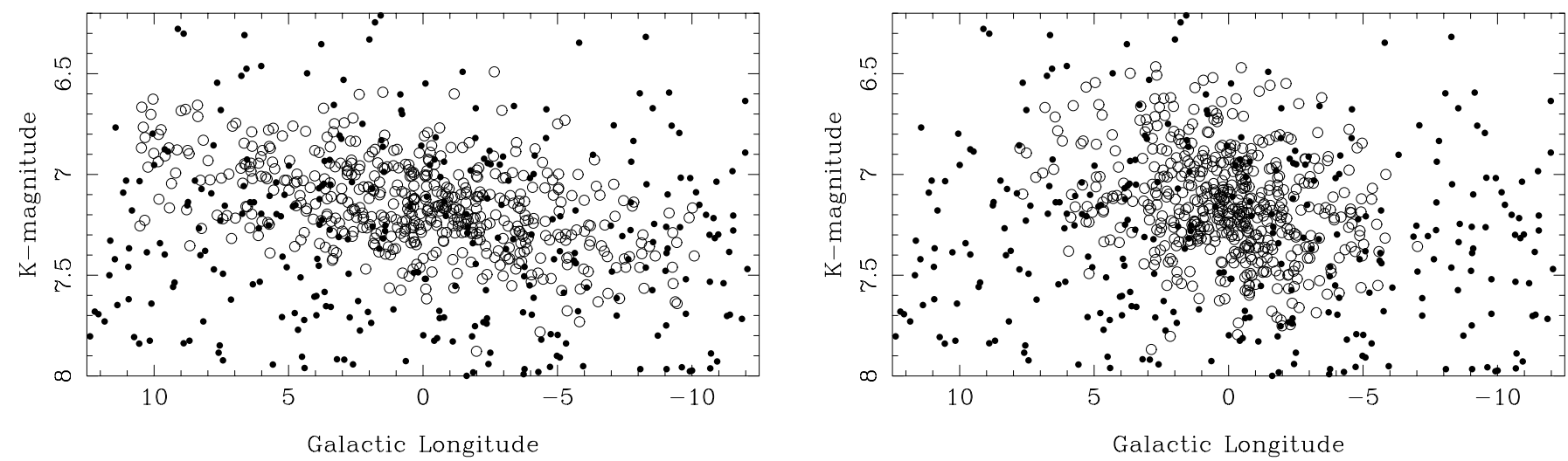

Fig. B.2. For angles of $\phi$ of 43 and 79 degrees (left and right panel), the distribution of $K$-magnitudes as a function of Galactic longitude for the stars shown in Fig. B.1. Disk stars are represented by filled circles, Bulge stars by open circles.
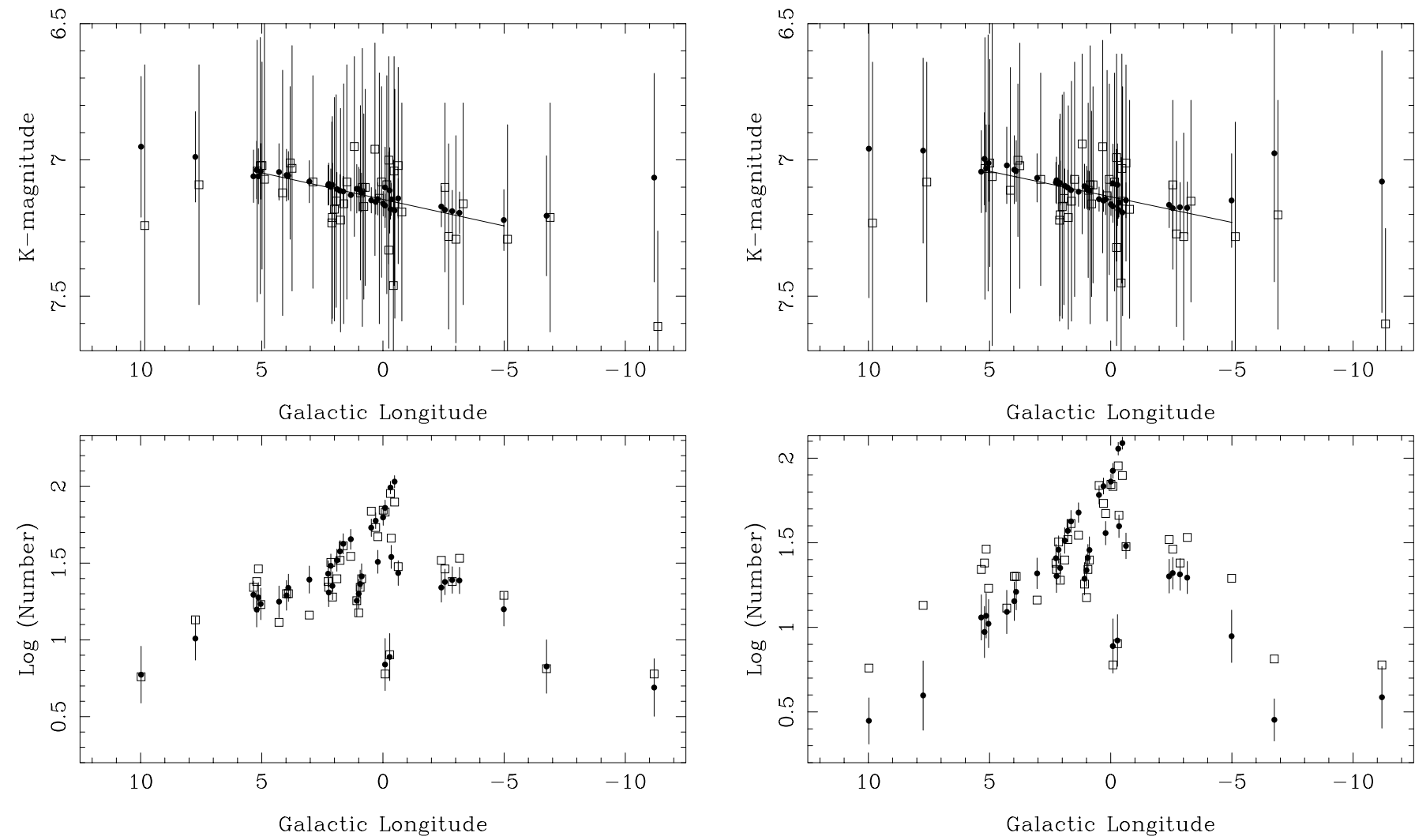

Fig. B.3. For angles of $\phi$ of 43 and 79 degrees (left and right panel), the top panel compares the observed (open squares) and modelled (filled circles) mean magnitudes and errors for the 40 fields listed in Table 6, with the line indicating the best fit over the range $|l|<5^{\circ}$. For clarity the observed and modelled points are slightly off-set in longitude. The bottom panel compares the observed (open squares) and modelled (filled circles) number of stars per field. For both angles the observed slope over the range $|l|<5^{\circ}$ is reproduced, but the distribution of stars is fitted much better for $\phi=43^{\circ}$.

For our comparison with the simulations, we used the solar metallicity models, which are expected to be the most representative for our Bulge sample. However, different studies indicate that the Bulge may have quite a broad metallicity distribution, peaking at about -0.25 dex with dispersion of 0.3 dex (see e.g. McWilliam \& Rich 1994; Ramirez et al. 2000). The AGB lifetime, LPV lifetime, and LPV period distribution was determined. The AGB lifetime is defined as the time between the first model in the file (the start of the AGB) up to the point where the remaining envelope mass becomes less than
$0.04 M_{\odot}$, or $T_{\text {eff }}>4500 \mathrm{~K}$, which is taken as the start of the post-AGB evolution.

For each timestep, the fundamental period is calculated following VW. The star is assumed to be in the Mira instability strip when the bolometric magnitude is within 0.20 mag (the assumed width of the instability strip at a given period) of the PL-relation (Feast et al. 1989)

$M_{\mathrm{bol}}=-3.00 \log P+2.85$ 
M. A. T. Groenewegen and J. A. D. L. Blommaert: Mira variables in the bulge, Online Material p 5

Table B.1. Observed total, and predicted number of disk and bulge stars per field.

\begin{tabular}{|c|c|c|c|c|c|c|c|c|}
\hline \multirow[b]{2}{*}{$l$} & \multirow[b]{2}{*}{$b$} & \multirow[b]{2}{*}{$N_{\text {obs }}$} & \multicolumn{2}{|c|}{$\phi=43^{\circ}$} & \multicolumn{2}{|c|}{$\phi=79^{\circ}$} & \multicolumn{2}{|c|}{$\phi=40^{\circ}, f_{\mathrm{h}}=1$} \\
\hline & & & $N_{\text {disk }}^{\prime}$ & $N_{\text {bulge }}$ & $N_{\text {disk }}$ & $N_{\text {bulge }}$ & $N_{\text {disk }}$ & $N_{\text {bulge }}$ \\
\hline 9.98 & -3.75 & 5.75 & $2.1 \pm 1.5$ & $3.1 \pm 2.0$ & $2.8 \pm 0.9$ & $0.0 \pm 0.0$ & $2.0 \pm 1.5$ & $3.9 \pm 1.9$ \\
\hline 7.74 & -3.50 & 13.5 & $3.1 \pm 1.6$ & $6.8 \pm 2.6$ & $2.8 \pm 1.6$ & $1.8 \pm 1.2$ & $3.0 \pm 1.7$ & $7.5 \pm 2.8$ \\
\hline 5.34 & +2.59 & 22 & $5.6 \pm 2.4$ & $14.0 \pm 3.5$ & $4.0 \pm 1.9$ & $7.4 \pm 2.6$ & $4.2 \pm 2.2$ & $15.4 \pm 3.9$ \\
\hline 5.21 & -3.50 & 24 & $3.8 \pm 1.7$ & $12.0 \pm 3.6$ & $2.9 \pm 1.7$ & $6.6 \pm 2.5$ & $2.9 \pm 1.7$ & $12.9 \pm 3.6$ \\
\hline 5.14 & +2.73 & 29 & $5.0 \pm 2.3$ & $13.9 \pm 3.4$ & $3.8 \pm 1.8$ & $7.9 \pm 2.8$ & $3.9 \pm 2.0$ & $15.3 \pm 4.0$ \\
\hline 5.04 & -3.37 & 17 & $3.9 \pm 2.0$ & $13.0 \pm 3.5$ & $2.9 \pm 1.7$ & $7.4 \pm 2.7$ & $3.0 \pm 2.0$ & $13.9 \pm 3.5$ \\
\hline 4.29 & -3.52 & 13 & $3.8 \pm 1.8$ & $14.0 \pm 3.6$ & $2.8 \pm 1.7$ & $9.5 \pm 3.0$ & $2.9 \pm 1.5$ & $15.2 \pm 3.7$ \\
\hline 3.98 & -3.41 & 20 & $3.9 \pm 2.0$ & $15.5 \pm 4.1$ & $2.9 \pm 1.8$ & $11.2 \pm 3.4$ & $3.0 \pm 1.8$ & $16.6 \pm 4.0$ \\
\hline 3.90 & -3.19 & 20 & $4.8 \pm 2.2$ & $17.2 \pm 4.0$ & $3.7 \pm 1.7$ & $12.7 \pm 3.4$ & $3.7 \pm 1.8$ & $18.7 \pm 4.1$ \\
\hline 3.04 & -3.18 & 14.5 & $4.9 \pm 2.2$ & $19.9 \pm 4.4$ & $3.8 \pm 1.7$ & $17.1 \pm 4.1$ & $3.8 \pm 1.7$ & $21.1 \pm 4.7$ \\
\hline 2.24 & -3.72 & 22 & $3.0 \pm 1.9$ & $16.9 \pm 4.1$ & $2.1 \pm 1.5$ & $17.4 \pm 4.1$ & $2.0 \pm 1.5$ & $18.3 \pm 3.9$ \\
\hline 2.26 & -3.22 & 24 & $4.9 \pm 2.3$ & $21.7 \pm 4.5$ & $3.8 \pm 2.0$ & $21.8 \pm 4.4$ & $3.1 \pm 1.9$ & $23.5 \pm 4.7$ \\
\hline 2.15 & -3.01 & 32 & $5.7 \pm 2.2$ & $24.9 \pm 5.0$ & $4.0 \pm 1.9$ & $24.5 \pm 4.8$ & $3.9 \pm 1.8$ & $26.5 \pm 4.8$ \\
\hline 2.08 & -3.59 & 19 & $3.9 \pm 1.9$ & $18.5 \pm 4.3$ & $2.9 \pm 1.7$ & $19.4 \pm 4.3$ & $2.8 \pm 1.5$ & $19.9 \pm 4.5$ \\
\hline 1.90 & -2.90 & 25 & $6.1 \pm 2.5$ & $26.7 \pm 5.0$ & $4.7 \pm 2.1$ & $27.9 \pm 5.1$ & $4.1 \pm 2.0$ & $28.7 \pm 5.0$ \\
\hline 1.77 & -2.70 & 33 & $7.1 \pm 2.6$ & $30.4 \pm 5.4$ & $5.2 \pm 2.1$ & $31.6 \pm 5.4$ & $5.0 \pm 2.3$ & $32.2 \pm 5.4$ \\
\hline 1.64 & -2.52 & 41 & $8.3 \pm 2.9$ & $33.8 \pm 5.6$ & $6.0 \pm 2.4$ & $36.1 \pm 6.0$ & $5.8 \pm 2.2$ & $35.9 \pm 6.0$ \\
\hline 1.33 & -2.43 & 35 & $8.8 \pm 2.8$ & $36.6 \pm 6.1$ & $6.6 \pm 2.5$ & $41.0 \pm 6.3$ & $5.9 \pm 2.5$ & $39.1 \pm 5.9$ \\
\hline 1.08 & -4.14 & 18 & $2.2 \pm 1.5$ & $15.3 \pm 3.8$ & $1.9 \pm 1.3$ & $17.4 \pm 4.0$ & $1.9 \pm 1.3$ & $16.5 \pm 3.9$ \\
\hline 0.94 & -3.69 & 22 & $3.7 \pm 1.9$ & $19.6 \pm 4.4$ & $2.8 \pm 1.6$ & $22.9 \pm 4.6$ & $2.0 \pm 1.5$ & $21.0 \pm 4.2$ \\
\hline 1.00 & -3.96 & 15 & $2.9 \pm 1.7$ & $17.0 \pm 4.0$ & $2.0 \pm 1.4$ & $19.6 \pm 4.3$ & $2.0 \pm 1.3$ & $18.1 \pm 4.1$ \\
\hline 0.88 & -3.49 & 25 & $4.0 \pm 1.9$ & $21.7 \pm 4.6$ & $3.0 \pm 1.7$ & $25.4 \pm 4.8$ & $2.9 \pm 1.7$ & $23.4 \pm 4.5$ \\
\hline 0.48 & -2.26 & 69 & $10.1 \pm 3.0$ & $43.3 \pm 6.4$ & $7.5 \pm 2.6$ & $53.2 \pm 7.2$ & $6.9 \pm 2.6$ & $46.0 \pm 6.7$ \\
\hline 0.30 & -2.10 & 54 & $11.6 \pm 3.1$ & $48.1 \pm 6.3$ & $8.6 \pm 2.8$ & $59.6 \pm 7.2$ & $7.9 \pm 2.7$ & $51.0 \pm 6.6$ \\
\hline 0.21 & +2.84 & 47 & $5.0 \pm 2.4$ & $26.9 \pm 5.0$ & $3.9 \pm 2.1$ & $31.8 \pm 5.2$ & $3.7 \pm 1.7$ & $28.2 \pm 5.1$ \\
\hline-0.01 & -2.02 & 70 & $12.0 \pm 3.5$ & $50.6 \pm 6.4$ & $9.0 \pm 2.9$ & $63.6 \pm 7.0$ & $8.5 \pm 2.7$ & $53.6 \pm 6.8$ \\
\hline-0.09 & -1.81 & 68 & $14.2 \pm 3.6$ & $57.8 \pm 7.4$ & $10.7 \pm 3.0$ & $73.3 \pm 8.1$ & $10.1 \pm 3.1$ & $61.6 \pm 7.5$ \\
\hline-0.09 & -5.86 & 6 & $1.0 \pm 0.8$ & $6.0 \pm 2.4$ & $0.9 \pm 0.7$ & $7.0 \pm 2.5$ & $0.0 \pm 0.5$ & $6.3 \pm 2.6$ \\
\hline-0.31 & -1.39 & 90 & $20.7 \pm 4.4$ & $77.4 \pm 8.5$ & $15.2 \pm 3.8$ & $98.4 \pm 9.7$ & $14.8 \pm 3.8$ & $81.9 \pm 8.6$ \\
\hline-0.28 & -5.70 & 8 & $1.0 \pm 0.8$ & $6.9 \pm 2.6$ & $0.9 \pm 0.7$ & $7.7 \pm 2.8$ & $0.0 \pm 0.7$ & $7.1 \pm 2.6$ \\
\hline-0.34 & -3.01 & 46 & $5.8 \pm 2.2$ & $29.1 \pm 5.4$ & $4.2 \pm 2.0$ & $35.0 \pm 5.5$ & $3.9 \pm 1.8$ & $30.4 \pm 5.6$ \\
\hline-0.48 & -1.26 & 79 & $23.3 \pm 4.7$ & $83.5 \pm 8.8$ & $17.4 \pm 4.1$ & $105.5 \pm 9.9$ & $16.7 \pm 4.0$ & $88.6 \pm 9.0$ \\
\hline-0.63 & -3.44 & 30 & $4.0 \pm 1.9$ & $22.9 \pm 4.5$ & $3.0 \pm 1.8$ & $27.1 \pm 5.0$ & $2.9 \pm 1.6$ & $24.4 \pm 4.9$ \\
\hline-2.41 & -3.64 & 33 & $3.1 \pm 1.7$ & $18.3 \pm 4.2$ & $2.1 \pm 1.5$ & $17.5 \pm 3.9$ & $2.1 \pm 1.5$ & $19.8 \pm 4.4$ \\
\hline-2.56 & -3.46 & 29 & $3.9 \pm 2.1$ & $19.9 \pm 4.1$ & $2.9 \pm 1.7$ & $17.9 \pm 4.1$ & $2.9 \pm 1.7$ & $21.2 \pm 4.6$ \\
\hline-2.86 & -3.35 & 24 & $4.1 \pm 2.1$ & $20.1 \pm 4.2$ & $3.0 \pm 1.9$ & $17.1 \pm 4.2$ & $3.0 \pm 1.8$ & $21.2 \pm 4.5$ \\
\hline-3.16 & -3.27 & 34 & $4.3 \pm 2.0$ & $19.8 \pm 4.3$ & $3.1 \pm 1.7$ & $16.1 \pm 4.1$ & $3.0 \pm 1.8$ & $21.4 \pm 4.5$ \\
\hline-4.99 & -3.58 & 19.5 & $3.1 \pm 1.7$ & $12.2 \pm 3.5$ & $2.8 \pm 1.6$ & $6.1 \pm 2.6$ & $2.9 \pm 1.6$ & $13.5 \pm 3.5$ \\
\hline-6.75 & -4.55 & 6.5 & $1.9 \pm 1.2$ & $4.9 \pm 2.2$ & $2.0 \pm 1.0$ & $0.9 \pm 0.7$ & $1.0 \pm 1.0$ & $5.2 \pm 2.1$ \\
\hline-11.19 & -2.78 & 6.0 & $4.9 \pm 2.0$ & $0.0 \pm 0.0$ & $3.9 \pm 1.5$ & $0.0 \pm 0.0$ & $5.0 \pm 2.0$ & $0.0 \pm 0.5$ \\
\hline
\end{tabular}

when assuming a LMC distance of 18.50 and when the mass loss rate is below a critical value, as this $P L$-relation was derived for optically visible objects and the LPV samples studied in G04 and in the present paper have been culled by only considering objects with $(J-K)_{0}<2$.0. In such a way the lifetime and the period distribution of optically visible LPVs can be determined.

The critical mass loss rate is determined by taking typical values of luminosity and effective temperature inside the instability strip for each mass in the grid of solar metallicities and then using a radiative transfer program (Groenewegen 1993) with the appropriate model atmosphere for M-stars (Fluks et al. 1994), and typical dust properties (silicate dust, condensation temperature of $1500 \mathrm{~K}$, dust-to-gas ratio 0.005 , expansion velocity $10 \mathrm{~km} \mathrm{~s}^{-1}$ ) to determine the critical mass loss rate at which the star would become redder in $(J-K)$ than 2.0. The critical mass loss rates found were between 4 and $20 \times 10^{-6} M_{\odot} \mathrm{yr}^{-1}$ depending on the initial mass of the model. In fact, the critical mass loss rate was observed to scale with $\sqrt{L}$, as expected as the dust optical depth predominantly determines the infrared colours; hence, the mass loss rate is proportional to the stellar radius, all other things being equal. For the 1.0 and $1.5 M_{\odot}$ initial mass models, the mass loss rate inside the instability strip always remains below the critical mass loss rate. The adopted critical mass loss rate is $1.0 \times 10^{-5} \sqrt{L / 13000} M_{\odot} \mathrm{yr}^{-1}$. The results for the solar metallicty models are summarised in Table C.1.

We now consider the synthetic AGB models of Wagenhuber \& Groenewegen (1998; WG), first because the VW models exist only for a limited number of initial 
M. A. T. Groenewegen and J. A. D. L. Blommaert: Mira variables in the bulge, Online Material p 6

Table C.1. AGB lifetime and optically visible LPV lifetimes for two sets of models.

\begin{tabular}{|c|c|c|c|c|c|c|c|}
\hline \multirow[b]{2}{*}{$Z$} & \multirow[b]{2}{*}{$\begin{array}{l}\text { Mass } \\
\left(M_{\odot}\right)\end{array}$} & \multicolumn{3}{|c|}{ Vassiliadis \& Wood } & \multicolumn{3}{|c|}{ Wagenhuber \& Groenewegen } \\
\hline & & $\begin{array}{c}\text { MS lifetime } \\
\left(10^{9} \text { years }\right)\end{array}$ & $\begin{array}{c}\text { AGB lifetime } \\
\left(10^{3} \text { years }\right)\end{array}$ & $\begin{array}{l}\text { LPV lifetime } \\
\left(10^{3} \text { years }\right)\end{array}$ & $\begin{array}{c}\text { AGB lifetime } \\
\left(10^{3} \text { years }\right)\end{array}$ & $\begin{array}{l}\text { LPV lifetime } \\
\left(10^{3} \text { years }\right)\end{array}$ & Remark \\
\hline \multirow[t]{3}{*}{0.016} & 1.0 & 11.25 & 595 & 101 & 487 & 49 & $\eta_{\mathrm{RGB}}=0, \alpha=1.9$ \\
\hline & & & & & 560 & 93 & $\eta_{\mathrm{RGB}}=0, \alpha=2.0$ \\
\hline & & & & & 595 & 129 & $\eta_{\mathrm{RGB}}=0, \alpha=2.1$ \\
\hline \multirow[t]{3}{*}{0.016} & 1.5 & 2.742 & 929 & 272 & 873 & 303 & $\eta_{\mathrm{RGB}}=0, \alpha=1.9$ \\
\hline & & & & & 942 & 284 & $\eta_{\mathrm{RGB}}=0, \alpha=2.0$ \\
\hline & & & & & 1019 & 282 & $\eta_{\mathrm{RGB}}=0, \alpha=2.1$ \\
\hline \multirow[t]{2}{*}{0.016} & 2.0 & 1.236 & 1168 & 153 & 1596 & 253 & $\eta_{\mathrm{RGB}}=0, \alpha=2.0$ \\
\hline & & & & & 1684 & 222 & $\eta_{\mathrm{RGB}}=0, \alpha=2.1$ \\
\hline \multirow[t]{2}{*}{0.016} & 2.5 & 0.619 & 2291 & 8 & 2284 & 84 & $\eta_{\mathrm{RGB}}=0, \alpha=2.0$ \\
\hline & & & & & 2354 & 50 & $\eta_{\mathrm{RGB}}=0, \alpha=2.1$ \\
\hline \multirow[t]{2}{*}{0.016} & 3.5 & 0.231 & 543 & 0 & 497 & 0 & $\eta_{\mathrm{RGB}}=0, \alpha=2.0$ \\
\hline & & & & & 542 & 0 & $\eta_{\mathrm{RGB}}=0, \alpha=2.1$ \\
\hline \multirow[t]{2}{*}{0.016} & 5.0 & 0.096 & 673 & 0 & 175 & 0 & $\eta_{\mathrm{RGB}}=0, \alpha=2.0$ \\
\hline & & & & & 174 & 0 & $\eta_{\mathrm{RGB}}=0, \alpha=2.1$ \\
\hline
\end{tabular}

masses and second because mass loss on the RGB was only included for initial masses below $1 M_{\odot}$, while it is well known that the effect of mass loss on the RGB is substantial also at and above $1 M_{\odot}$. The VW mass loss rate recipe was implemented; and to mimic the VW tracks as closely as possible, the mixing length parameter $\alpha$ (basically setting the effective temperature scale) in the WG models was tuned to give similar AGB and LPV lifetimes. The results are summarised in Table C.1. It turns out that with $\alpha=2.0$ the lifetimes match very well especially at low initial mass.

Mass loss on the RGB is described by a Reimers law with a scaling factor $\eta_{\mathrm{RGB}}=0.35$. This gives the required mass loss $\left(0.13,0.16,0.17 M_{\odot}\right.$ for a star of $1.0 M_{\odot}$ initial mass at $Z=0.004,0.008,0.019$, respectively; M. Salaris, private communication) to give the observed mean colour of Horizontal Branch stars in Galactic Globular Clusters. Table C.2 summarises the AGB and LPV lifetime for a set of WG models with $\eta_{\mathrm{RGB}}=0.35$ and $\alpha=2.0$, and Fig. C. 1 displays the period distribution of optically visible stars inside the instability strip (normalised to one each time) for a few initial masses.

Table C. 2 also includes the results for a few initial masses if slightly different metallicities of $Z=0.01$ and 0.02 are adopted, and Fig. C. 2 shows the corresponding Period distribution. These results indicate that the effect of metallicity on the pulsation properties for the typical metallicities in the Bulge is small.
Table C.2. AGB lifetime and optically visible LPV lifetimes for the final set of synthetic models, with $\eta_{\mathrm{RGB}}=0.35$ and $\alpha=2.0$.

\begin{tabular}{rrrr}
\hline \hline$Z$ & $\begin{array}{r}\text { Mass } \\
\left(M_{\odot}\right)\end{array}$ & $\begin{array}{r}\text { AGB lifetime } \\
\left(10^{3} \text { years }\right)\end{array}$ & $\begin{array}{r}\text { LPV lifetime } \\
\left(10^{3} \text { years }\right)\end{array}$ \\
\hline 0.016 & 0.85 & 240 & 1.6 \\
0.016 & 0.9 & 288 & 1.0 \\
0.016 & 1.0 & 305 & 1.5 \\
0.016 & 1.1 & 445 & 41. \\
0.010 & 1.2 & 644 & 132. \\
0.016 & 1.2 & 552 & 108. \\
0.020 & 1.2 & 528 & 95. \\
0.016 & 1.3 & 648 & 161. \\
0.016 & 1.4 & 770 & 247. \\
0.016 & 1.5 & 858 & 296. \\
0.016 & 1.6 & 965 & 293. \\
0.016 & 1.7 & 1103 & 282. \\
0.016 & 1.8 & 1211 & 296. \\
0.016 & 1.9 & 1362 & 289. \\
0.010 & 2.0 & 1610 & 234. \\
0.016 & 2.0 & 1522 & 269. \\
0.020 & 2.0 & 1527 & 283. \\
0.016 & 2.1 & 1711 & 250. \\
0.016 & 2.2 & 1898 & 220. \\
0.016 & 2.3 & 2071 & 182. \\
0.016 & 2.4 & 2182 & 131. \\
0.016 & 2.5 & 2269 & 90. \\
0.016 & 2.6 & 2300 & 59. \\
0.016 & 2.7 & 2260 & 40. \\
0.016 & 2.8 & 2125 & 26. \\
0.016 & 2.9 & 1903 & 14. \\
0.010 & 3.0 & 1435 & 3. \\
0.016 & 3.0 & 1643 & 10. \\
0.020 & 3.0 & 1748 & 10. \\
0.016 & 3.1 & 1355 & 3. \\
0.016 & 3.4 & 641 & 0. \\
\hline & & &
\end{tabular}


M. A. T. Groenewegen and J. A. D. L. Blommaert: Mira variables in the bulge, Online Material $p 7$
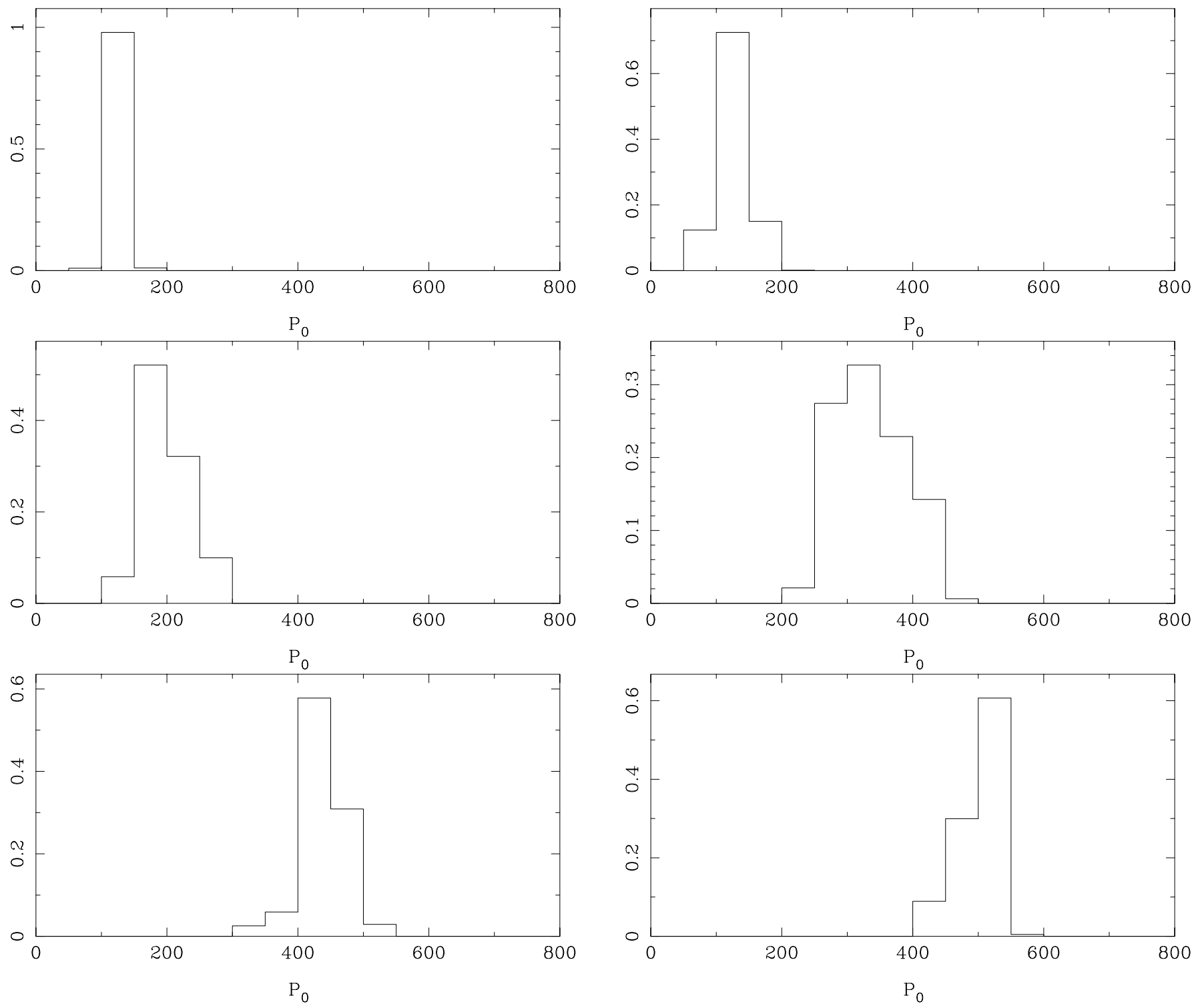

Fig. C.1. Theoretical period distribution of optically visible stars inside the observed instability strip for masses $1.1,1.2,1.5,2.0,2.5,3.0 M_{\odot}$ (left to right, top to bottom). 
M. A. T. Groenewegen and J. A. D. L. Blommaert: Mira variables in the bulge, Online Material $p 8$
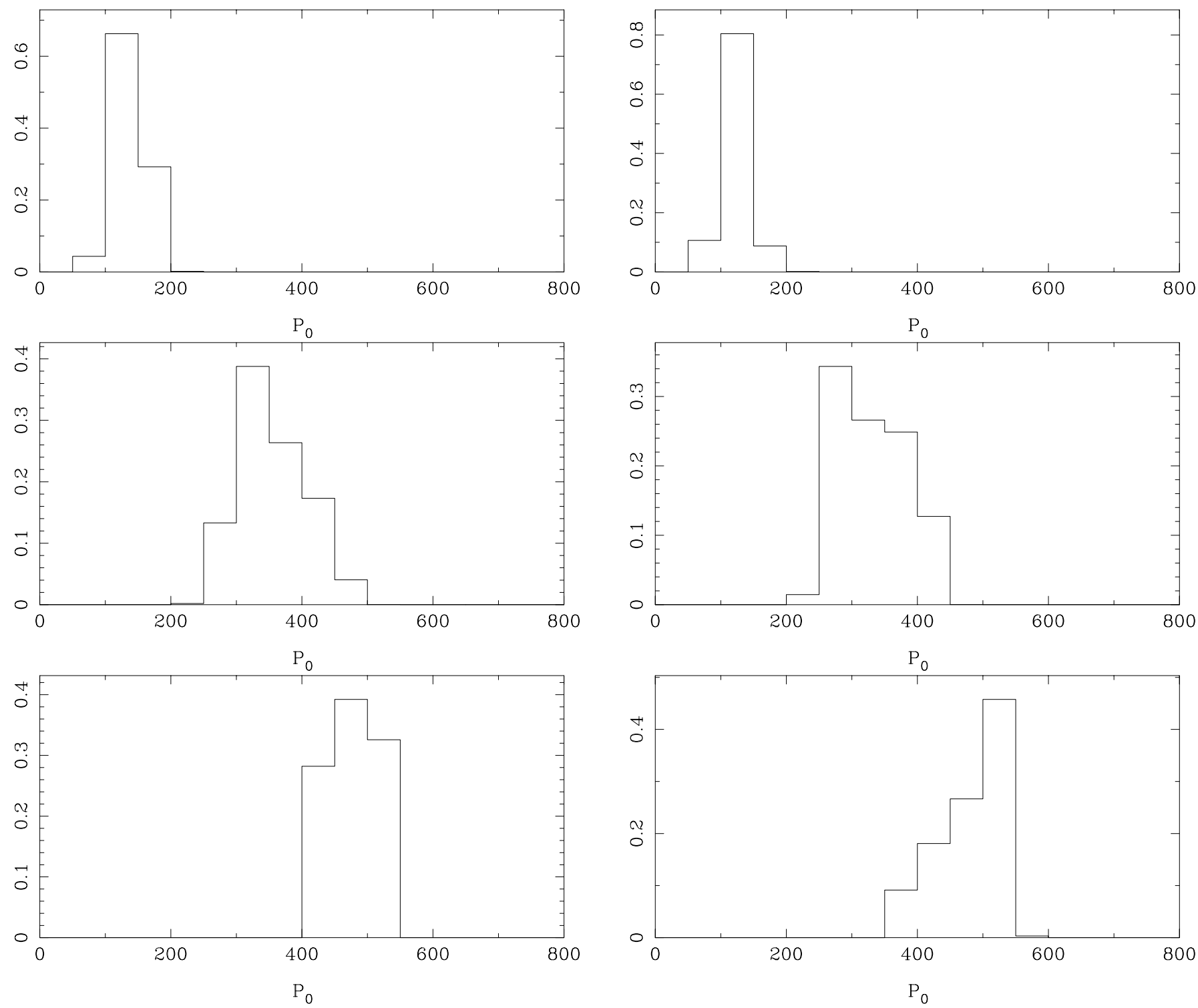

Fig. C.2. Like Fig. C.1 illustrating the influence of metallicity on the theoretical distribution. In the left hand panels for $Z=0.010$ and on the right for $Z=0.020$, for stars with inital masses 1.2 (top), 2.0 (middle) and 3.0 (bottom) $M_{\odot}$. 
M. A. T. Groenewegen and J. A. D. L. Blommaert: Mira variables in the bulge, Online Material $p 9$

Table 2. First entries in the electronically available table, which list: OGLE-field and number, other names, spectral type, and references. ISOGAL sources from the official catalog (OGA03) have the prefix "ISOGAL". ISOGAL sources studied in OOS03 have the prefix "OOS03". References in Col. 4 are given in the bibliography of the main text.

\begin{tabular}{|c|c|c|c|}
\hline OGLE-name & Other name & Chemical type & Remarks \\
\hline bul_sc01_0098 & \multirow[t]{9}{*}{ V $4713 \mathrm{Sgr}$} & & \\
\hline bul_sc01_0100 & & & \\
\hline bul_sc01_0103 & & & \\
\hline bul_sc01_0128 & & & \\
\hline bul_sc01_0177 & & & \\
\hline bul_sc01_0193 & & & \\
\hline bul_sc01_0235 & & & \\
\hline bul_sc01_0237 & & & \\
\hline bul_sc01_0388 & & & \\
\hline bul_sc01_0558 & \multirow[t]{8}{*}{ BW6_V1_MISC } & & \\
\hline bul_sc01_0602 & & & \\
\hline bul_sc01_0611 & & & \\
\hline bul_sc01_0878 & & & \\
\hline bul_sc01_0916 & & & \\
\hline bul_sc01_1241 & & & \\
\hline bul_sc01_1253 & & & \\
\hline bul_sc01_1254 & & & \\
\hline bul_sc01_1467 & \multirow{4}{*}{ BW5_V1_MISC } & & \\
\hline bul_sc01_1533 & & & \\
\hline bul_sc01_1561 & & & \\
\hline bul_sc01_1616 & & & \\
\hline bul_sc01_1738 & \multirow{4}{*}{ BW5_V4_MISC } & & \\
\hline bul_sc01_1818 & & & \\
\hline bul_sc01_1821 & & & \\
\hline bul_sc01_1944 & & & \\
\hline bul_sc01_2079 & \multirow[t]{5}{*}{ BW5_V2_MISC, V 3999 Sgr } & & \\
\hline bul_sc01_2222 & & & \\
\hline bul_sc01_2231 & & & \\
\hline bul_sc01_2303 & & & \\
\hline bul_sc01_2447 & & & \\
\hline bul_sc01_2542 & \multirow[t]{23}{*}{$\begin{array}{l}\text { TLE_NGC_6522_181, V } 1371 \text { Sgr, } \\
\text { MACHO } 119.19833 .209\end{array}$} & \multirow[t]{23}{*}{ M7 } & \multirow[t]{23}{*}{$\begin{array}{l}\text { GS03, Glass \& Feast (1982; GF82), } \\
\text { Lloyd-Evans (1976; hereafter LE76) }\end{array}$} \\
\hline bul_sc01_3024 & & & \\
\hline bul_sc01_3202 & & & \\
\hline bul_sc01_3212 & & & \\
\hline bul_sc01_3916 & & & \\
\hline bul_sc01_3955 & & & \\
\hline bul_sc01_4032 & & & \\
\hline bul_sc01_4178 & & & \\
\hline bul_sc01_4185 & & & \\
\hline bul_sc01_4194 & & & \\
\hline bul_sc01_4200 & & & \\
\hline bul_sc01_4350 & & & \\
\hline bul_sc02_0002 & & & \\
\hline bul_sc02_0033 & & & \\
\hline bul_sc02_0061 & & & \\
\hline bul_sc02_0080 & & & \\
\hline bul_sc02_0098 & & & \\
\hline bul_sc02_0136 & & & \\
\hline bul_sc02_0206 & & & \\
\hline bul_sc02_0341 & & & \\
\hline bul_sc02_0369 & & & \\
\hline bul_sc02_0409 & & & \\
\hline bul_sc02_0420 & & & \\
\hline
\end{tabular}


M. A. T. Groenewegen and J. A. D. L. Blommaert: Mira variables in the bulge, Online Material p 10

Table 2. continued.

\begin{tabular}{|c|c|c|c|}
\hline OGLE-name & Other name & Chemical type & Remarks \\
\hline bul_sc02_0529 & & & \\
\hline bul_sc02_0564 & & & \\
\hline bul_sc02_0574 & & & \\
\hline bul_sc02_0654 & & & \\
\hline bul_sc02_0748 & & & \\
\hline bul_sc02_1136 & & & \\
\hline bul_sc02_1214 & & & \\
\hline bul_sc02_1220 & & & \\
\hline bul_sc02_1331 & IRAS_18017-2907 & & \\
\hline bul_sc02_1492 & & & \\
\hline bul_sc02_1555 & & & \\
\hline bul_sc02_1561 & & & \\
\hline bul_sc02_1733 & & & \\
\hline bul_sc02_1985 & & & \\
\hline bul_sc02_2007 & & & \\
\hline bul_sc02_2276 & & & \\
\hline bul_sc02_2604 & & & \\
\hline bul_sc02_2755 & & & \\
\hline bul_sc02_2834 & & & \\
\hline bul_sc02_2842 & & & \\
\hline bul_sc02_2861 & & & \\
\hline bul_sc02_3055 & & & \\
\hline bul_sc02_3184 & & & \\
\hline bul_sc02_3258 & & & \\
\hline bul_scc02_3358 & & & \\
\hline bul_sc02_3360 & & & \\
\hline bul_sc02_3580 & & & \\
\hline bul_sc02_3721 & & & \\
\hline bul_sc02_3738 & & & \\
\hline bul_sc02_3778 & & & \\
\hline bul_sc02_3933 & & & \\
\hline bul_scc02_4290 & & & \\
\hline bul_sc02_4321 & & & \\
\hline bul_sc02_4772 & & & \\
\hline bul_sc02_4926 & & & \\
\hline bul_sc02_5217 & & & \\
\hline bul_sc02_5236 & & & \\
\hline bul_sc03_0047 & & & \\
\hline bul_sc03_0048 & & & \\
\hline bul_sc03_0057 & & & \\
\hline bul_sc03_0078 & & & \\
\hline bul_sc03_0114 & & & \\
\hline bul_sc03_0135 & & & \\
\hline bul_sc03_0191 & & & \\
\hline bul_sc03_0307 & & & \\
\hline bul_sc03_0318 & & & \\
\hline bul_sc03_0375 & & & \\
\hline bul_sc03_0689 & & & \\
\hline bul_sc03_0756 & & & \\
\hline bul_sc03_0773 & & & \\
\hline bul_sc03_0878 & & & \\
\hline bul_sc03_0897 & & & \\
\hline bul_sc03_0921 & & & \\
\hline bul_sc03_0926 & & & \\
\hline bul_sc03_1072 & & & \\
\hline bul_sc03_1082 & & & \\
\hline bul_sc03_1098 & & & \\
\hline bul_sc03_1105 & & & \\
\hline bul_sc03_1109 & & & \\
\hline
\end{tabular}


M. A. T. Groenewegen and J. A. D. L. Blommaert: Mira variables in the bulge, Online Material p 11

Table 2. continued.

\begin{tabular}{|c|c|c|c|}
\hline$\overline{\text { OGLE-name }}$ & Other name & Chemical type & Remarks \\
\hline \multicolumn{4}{|l|}{ bul_sc03_1272 } \\
\hline \multicolumn{4}{|l|}{ bul_sc03_1288 } \\
\hline \multicolumn{4}{|l|}{ bul_sc03_1393 } \\
\hline \multicolumn{4}{|l|}{ bul_sc03_1707 } \\
\hline \multicolumn{4}{|l|}{ bul_sc03_1788 } \\
\hline \multicolumn{4}{|l|}{ bul_sc03_1810 } \\
\hline \multicolumn{4}{|l|}{ bul_sc03_1833 } \\
\hline \multicolumn{4}{|l|}{ bul_sc03_1890 } \\
\hline \multicolumn{4}{|l|}{ bul_sc03_1952 } \\
\hline \multicolumn{4}{|l|}{ bul_sc03_2188 } \\
\hline \multicolumn{4}{|l|}{ bul_sc03_2334 } \\
\hline bul_sc03_2339 & ISOGAL-PJ175332.8-300746 & & \\
\hline bul_sc03_2487 & ISOGAL-PJ175336.4-300655 & & \\
\hline bul_sc03_2495 & ISOGAL-PJ175345.6-300709 & & \\
\hline bul_sc03_2532 & ISOGAL-PJ175358.1-300652 & & \\
\hline \multicolumn{4}{|l|}{ bul_sc03_2553 } \\
\hline \multicolumn{4}{|l|}{ bul_sc03_2695 } \\
\hline bul_sc03_2750 & ISOGAL-PJ175328.9-300529 & & \\
\hline bul_sc03_2751 & ISOGAL-PJ175330.7-300517 & & \\
\hline \multicolumn{4}{|l|}{ bul_sc03_2767 } \\
\hline \multirow{2}{*}{\multicolumn{4}{|c|}{$\begin{array}{l}\text { bul_sc03_2806 } \\
\text { bul_sc03_2855 }\end{array}$}} \\
\hline & & & \\
\hline bul_sc03_2862 & ISOGAL-PJ175319.0-300407 & & \\
\hline bul_sc03_2878 & ISOGAL-PJ175327.8-300410 & & \\
\hline bul_sc03_3023 & ISOGAL-PJ175331.3-300330 & & \\
\hline bul_sc03_3086 & ISOGAL-PJ175403.2-300321 & & \\
\hline bul_sc03_3177 & ISOGAL-PJ175335.7-300255 & & \\
\hline bul_sc03_3205 & ISOGAL-PJ175355.6-300231 & & \\
\hline bul_sc03_3346 & ISOGAL-PJ175306.1-300103 & & \\
\hline bul_sc03_3351 & ISOGAL-PJ175310.0-300037 & & \\
\hline \multicolumn{4}{|l|}{ bul_sc03_3552 } \\
\hline bul_sc03_3686 & ISOGAL-PJ175336.6-295916 & & \\
\hline \multicolumn{4}{|l|}{ bul_sc03 3710} \\
\hline bul_sc03_3712 & & & \\
\hline bul_sc03_3714 & & & \\
\hline bul_sc03_3870 & & & \\
\hline bul_sc03_3941 & ISOGAL-PJ175332.6-295712 & & \\
\hline bul_sc03_3951 & & & \\
\hline bul_sc03_4046 & ISOGAL-PJ175327.1-295643 & & \\
\hline bul_sc03_4235 & & & \\
\hline bul_sc03_4419 & & & \\
\hline bul_sc03_4426 & IRAS_17502-2953 & & \\
\hline bul_sc03_4494 & & & \\
\hline bul_sc03_4522 & & & \\
\hline bul_sc03_4551 & & & \\
\hline bul_sc03_4588 & & & \\
\hline bul_sc03_4629 & & & \\
\hline bul_sc03_4644 & & & \\
\hline bul_sc03_4774 & & & \\
\hline bul_sc03_4784 & & & \\
\hline bul_sc03_4865 & & & \\
\hline bul_sc03_5306 & & & \\
\hline bul_sc03_5364 & & & \\
\hline bul_sc03_5383 & & & \\
\hline bul_sc03_5408 & & & \\
\hline bul_sc03_5414 & & & \\
\hline bul_sc03_5426 & & & \\
\hline bul_sc03_5703 & & & \\
\hline bul_sc03_5877 & & & \\
\hline
\end{tabular}


M. A. T. Groenewegen and J. A. D. L. Blommaert: Mira variables in the bulge, Online Material p 12

Table 2. continued.

\begin{tabular}{|c|c|c|c|}
\hline \multirow{2}{*}{$\begin{array}{l}\text { OGLE-name } \\
\text { bul_sc03_5929 }\end{array}$} & Other name & Chemical type & Remarks \\
\hline & & & \\
\hline bul_sc03 6083 & & & \\
\hline bul_sc03_6107 & & & \\
\hline bul_sc03_6205 & & & \\
\hline bul_sc03_6207 & & & \\
\hline bul_sc03_6208 & & & \\
\hline bul_sc03_6508 & & & \\
\hline bul_sc03_6587 & & & \\
\hline bul_sc03_6668 & & & \\
\hline bul_sc03_6711 & & & \\
\hline bul_sc03_6756 & & & \\
\hline bul_sc03_6997 & & & \\
\hline bul_sc03_7010 & & & \\
\hline bul_sc03_7068 & & & \\
\hline bul_sc03_7129 & & & \\
\hline bul_sc03_7147 & & & \\
\hline bul_sc03_7270 & & & \\
\hline bul_sc03_7348 & & & \\
\hline bul_sc03_7487 & & & \\
\hline bul_sc03 7491 & & & \\
\hline bul_sc03_7505 & & & \\
\hline bul_sc03_7572 & & & \\
\hline bul_sc03_7614 & & & \\
\hline bul_sc03_7694 & & & \\
\hline bul_sc03_7733 & & & \\
\hline bul_sc03_7775 & & & \\
\hline bul_sc03_8032 & & & \\
\hline bul_sc03_8070 & & & \\
\hline bul_sc03_8185 & & & \\
\hline bul_sc03_8195 & IRAS_17507-2931 & & \\
\hline bul_sc03_8201 & [DB2000] 16 & & Dutra \& Bica (2000) \\
\hline bul_sc03_8207 & & & \\
\hline bul_sc03_8374 & & & \\
\hline bul_sc03_8429 & & & \\
\hline bul_sc04_0040 & & & \\
\hline bul_sc04_0047 & & & \\
\hline bul_sc04_0197 & & & \\
\hline bul_sc04_0352 & & & \\
\hline bul_sc04_0375 & ISOGAL-PJ175405.7-300902 & & \\
\hline bul_sc04_0408 & & & \\
\hline bul_sc04_0429 & & & \\
\hline bul_sc04_0456 & & & \\
\hline bul_sc04_0478 & & & \\
\hline bul_sc04_0585 & & & \\
\hline bul_sc04_0678 & & & \\
\hline bul_sc04_1100 & & & \\
\hline bul_sc04_1490 & & & \\
\hline bul_sc04_1491 & & & \\
\hline bul_sc04_1616 & & & \\
\hline bul_sc04_1688 & & & \\
\hline bul_sc04_1709 & & & \\
\hline bul_sc04_1817 & & & \\
\hline bul_sc04_1841 & & & \\
\hline bul_sc04_1842 & & & \\
\hline bul_sc04_1895 & & & \\
\hline bul_sc04_1972 & & & \\
\hline bul_sc04_1992 & & & \\
\hline bul_sc04_2209 & & & \\
\hline bul $\mathrm{sc} 042303$ & & & \\
\hline
\end{tabular}


M. A. T. Groenewegen and J. A. D. L. Blommaert: Mira variables in the bulge, Online Material p 13

Table 2. continued.

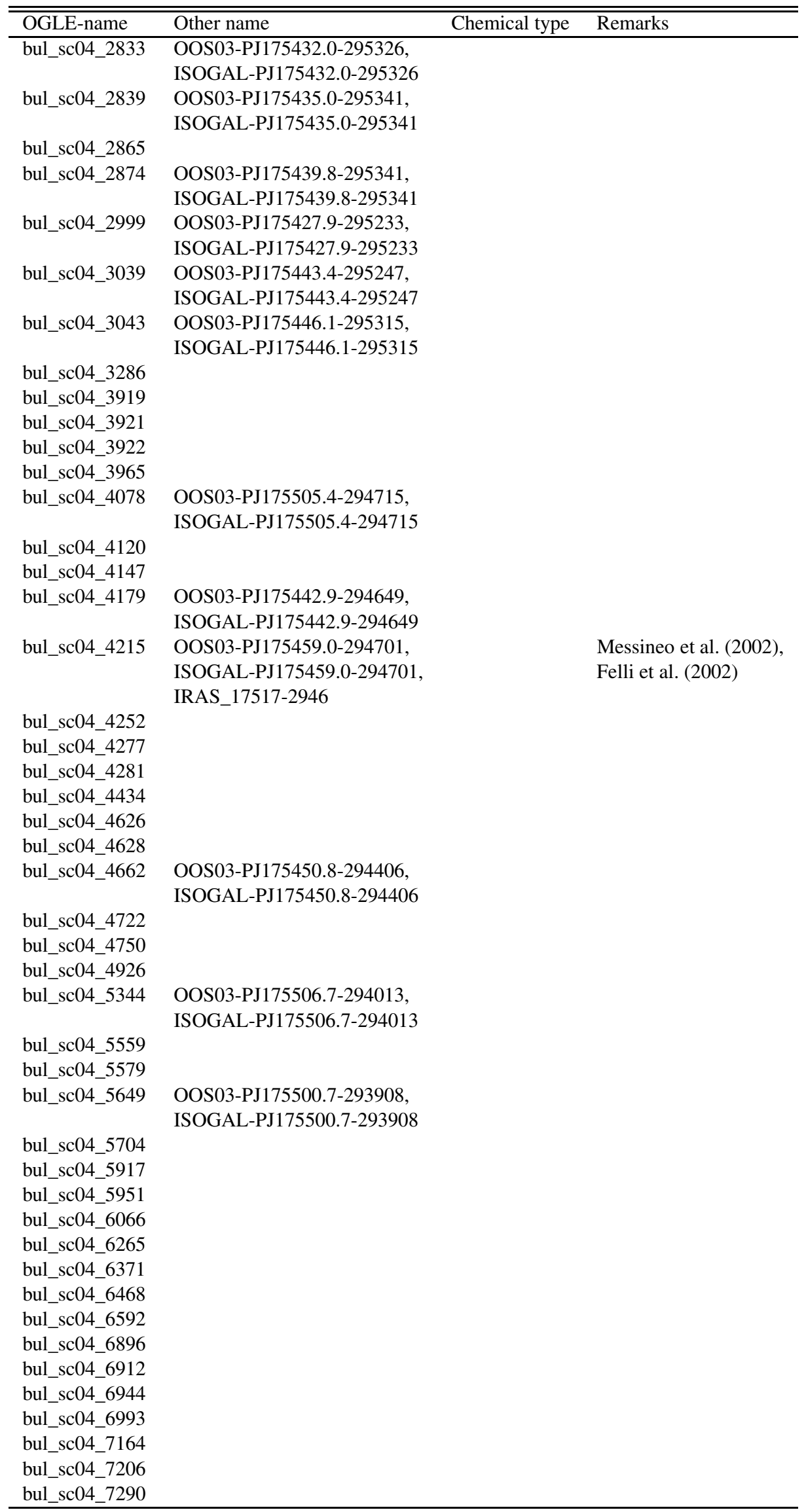


M. A. T. Groenewegen and J. A. D. L. Blommaert: Mira variables in the bulge, Online Material p 14

Table 2. continued.

\begin{tabular}{|c|c|c|c|}
\hline OGLE-name & Other name & Chemical type & Remarks \\
\hline \multicolumn{4}{|l|}{ bul_sc04_7534 } \\
\hline \multicolumn{4}{|l|}{ bul_sc04_7541 } \\
\hline \multicolumn{4}{|l|}{ bul_sc04_7691 } \\
\hline \multicolumn{4}{|l|}{ bul_sc04_8070 } \\
\hline \multicolumn{4}{|l|}{ bul_sc04_8241 } \\
\hline \multicolumn{4}{|l|}{ bul_sc04_8585 } \\
\hline \multicolumn{4}{|l|}{ bul_sc04_8592 } \\
\hline \multicolumn{4}{|l|}{ bul_sc04_8667 } \\
\hline \multicolumn{4}{|l|}{ bul_sc04_8749 } \\
\hline \multicolumn{4}{|l|}{ bul_sc04_8786 } \\
\hline \multicolumn{4}{|l|}{ bul_sc04_8831 } \\
\hline \multicolumn{4}{|l|}{ bul_sc04_8886 } \\
\hline \multicolumn{4}{|l|}{ bul_sc04_8891 } \\
\hline \multicolumn{4}{|l|}{ bul_sc04_9008 } \\
\hline \multicolumn{4}{|l|}{ bul_sc04_9056 } \\
\hline \multicolumn{4}{|l|}{ bul_sc05_0084 } \\
\hline \multicolumn{4}{|l|}{ bul_sc05_0108 } \\
\hline bul_sc05_0118 & & & \\
\hline bul_sc05_0126 & & & \\
\hline bul_sc05_0260 & V 2187 & & Terzan \& Ounnas (1988; TO88) \\
\hline bul_sc05_0277 & & & \\
\hline bul_sc05_0347 & & & \\
\hline bul_sc05_0363 & & & \\
\hline bul_sc05_0364 & & & \\
\hline bul_sc05_0367 & & & \\
\hline bul_sc05_0373 & & & \\
\hline bul_sc05_0479 & & & \\
\hline bul_sc05_0513 & & & \\
\hline bul_sc05_0519 & & & \\
\hline bul_sc05_0638 & & & \\
\hline bul_sc05_0903 & & & \\
\hline bul_sc05_0918 & & & \\
\hline bul_sc05_0939 & & & \\
\hline bul_sc05_1006 & & & \\
\hline bul_sc05_1072 & & & \\
\hline bul_sc05_1133 & & & \\
\hline bul_sc05_1185 & & & \\
\hline bul_sc05_1304 & & & \\
\hline bul_sc05_1419 & & & \\
\hline bul_sc05_1533 & & & \\
\hline bul_sc05_1534 & & & \\
\hline bul_sc05_1584 & & & \\
\hline bul_sc05_1708 & V 2155 & & TO88 \\
\hline bul_sc05_1795 & & & \\
\hline bul_sc05_1843 & & & \\
\hline bul_sc05_1845 & & & \\
\hline bul_sc05_1894 & NGC 6451 PMR 775 & & Paunzen et al (2003) \\
\hline bul_sc05_1907 & & & \\
\hline bul_sc05_2051 & & & \\
\hline bul_sc05_2107 & & & \\
\hline bul_sc05_2132 & & & \\
\hline bul_sc05_2136 & & & \\
\hline bul_sc05_2152 & & & \\
\hline bul_sc05_2241 & V 2186 & & TO88 \\
\hline bul_sc05_2367 & & & \\
\hline bul_sc05_2524 & V 2162 & & TO88 \\
\hline bul_sc05_2556 & & & \\
\hline bul_sc05_2659 & & & \\
\hline bul_sc05_2677 & & & \\
\hline
\end{tabular}


M. A. T. Groenewegen and J. A. D. L. Blommaert: Mira variables in the bulge, Online Material p 15

Table 2. continued.

\begin{tabular}{|c|c|c|c|}
\hline OGLE-name & Other name & Chemical type & Remarks \\
\hline bul_sc05_2790 & & & \\
\hline bul_sc05 3021 & & & \\
\hline bul_sc05_3027 & & & \\
\hline bul_sc05_3050 & & & \\
\hline bul_sc05_3125 & & & \\
\hline bul_sc05_3330 & & & \\
\hline bul_sc05_3346 & & & \\
\hline bul_sc05_3414 & & & \\
\hline bul_sc05_3416 & & & \\
\hline bul_sc05_3431 & & & \\
\hline bul_sc05 3451 & & & \\
\hline bul_sc05_3601 & & & \\
\hline bul_sc05_3893 & & & \\
\hline bul_sc05_3894 & & & \\
\hline bul_sc05_3902 & & & \\
\hline bul_sc05_3949 & & & \\
\hline bul_sc05_4084 & & & \\
\hline bul_sc05_4093 & & & \\
\hline bul_sc05_4104 & & & \\
\hline bul_sc05_4161 & & & \\
\hline bul_sc05_4196 & & & \\
\hline bul_sc05_4269 & & & \\
\hline bul_sc05_4342 & & & \\
\hline bul_sc05_4354 & & & \\
\hline bul_sc05_4447 & & & \\
\hline bul_sc05_4498 & & & \\
\hline bul_sc05_4511 & & & \\
\hline bul_sc05_4531 & & & \\
\hline bul_sc05_4543 & & & \\
\hline bul_sc05_4598 & & & \\
\hline bul_sc05_4660 & & & \\
\hline bul_sc05_4765 & & & \\
\hline bul_sc05_4880 & & & \\
\hline bul_sc05_4911 & & & \\
\hline bul_sc05_4916 & & & \\
\hline bul_sc05_4968 & & & \\
\hline bul_sc05_5001 & & & \\
\hline bul_sc05_5003 & & & \\
\hline bul_sc05_5082 & & & \\
\hline bul_sc05_5088 & & & \\
\hline bul_sc05_5118 & & & \\
\hline bul_sc05_5226 & & & \\
\hline bul_sc05_5246 & & & \\
\hline bul_sc05_5247 & & & \\
\hline bul_sc05_5366 & & & \\
\hline bul_sc05_5391 & & & \\
\hline bul_sc05_5464 & & & \\
\hline bul_sc05_5473 & & & \\
\hline bul_sc05_5635 & & & \\
\hline bul_sc05_5748 & & & \\
\hline bul_sc05_5823 & & & \\
\hline bul_sc05_6058 & & & \\
\hline bul_sc05_6075 & & & \\
\hline bul_sc05_6188 & & & \\
\hline bul_sc05_6232 & & & \\
\hline bul_sc05_6393 & & & \\
\hline bul_sc05_6467 & & & \\
\hline bul_sc05_6478 & & & \\
\hline bul_sc05_6479 & & & \\
\hline
\end{tabular}


M. A. T. Groenewegen and J. A. D. L. Blommaert: Mira variables in the bulge, Online Material p 16

Table 2. continued.

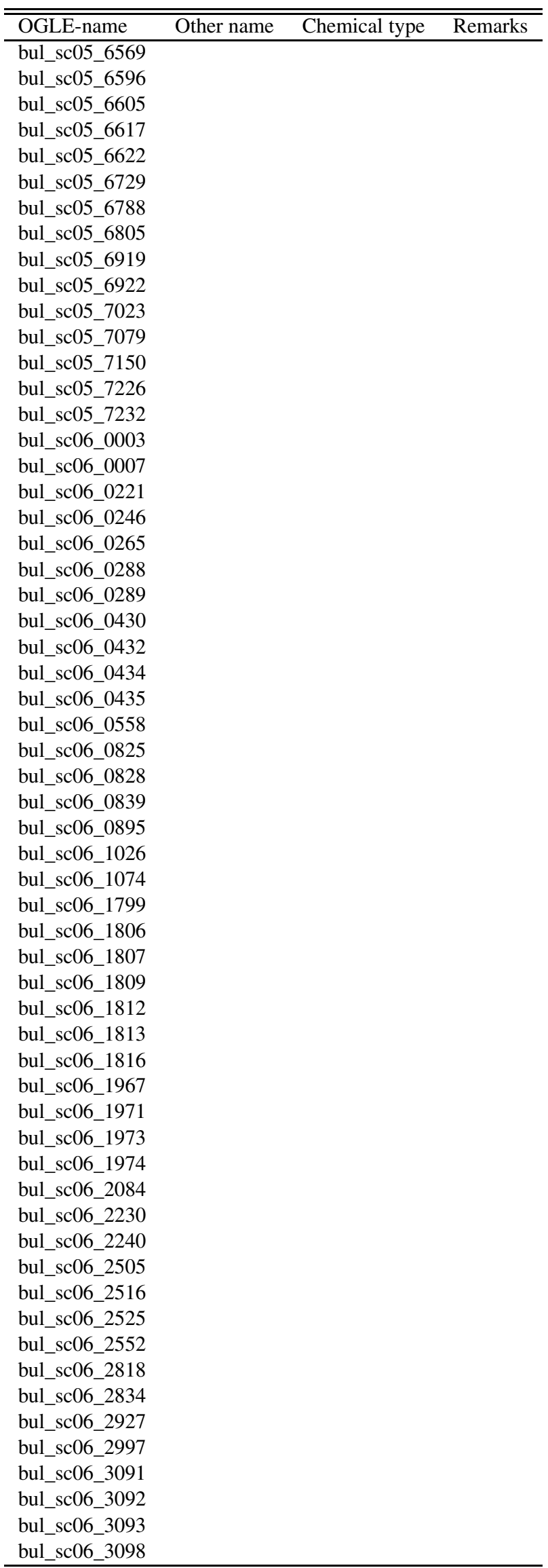


M. A. T. Groenewegen and J. A. D. L. Blommaert: Mira variables in the bulge, Online Material p 17

Table 2. continued.

\begin{tabular}{|c|c|c|c|}
\hline OGLE-name & Other name & Chemical type & Remarks \\
\hline bul_sc06_3099 & & & \\
\hline bul_sc06_3103 & & & \\
\hline bul_sc06_3195 & & & \\
\hline bul_sc07_0078 & & & \\
\hline bul_sc07_0081 & & & \\
\hline bul_sc07_0082 & & & \\
\hline bul_sc07_0083 & & & \\
\hline bul_sc07_0085 & & & \\
\hline bul_sc07_0175 & & & \\
\hline bul_sc07_0193 & & & \\
\hline bul_sc07_0202 & & & \\
\hline bul_sc07_0203 & & & \\
\hline bul_sc07_0413 & & & \\
\hline bul_sc07_0425 & IRAS_18059-3218 & & \\
\hline bul_sc07_0749 & & & \\
\hline bul_sc07_0750 & & & \\
\hline bul_sc07_1162 & & & \\
\hline bul_sc07_1267 & & & \\
\hline bul_sc07_1282 & & & \\
\hline bul_sc07_1464 & [DHM99] BE582 & & DMH99 \\
\hline bul_sc07_1469 & & & \\
\hline bul_sc07_1487 & & & \\
\hline bul_sc07_1491 & & & \\
\hline bul_sc07_1607 & IRAS_18061-3140 & & $\begin{array}{l}\text { Kwok et al. (1997), te Lintel Hekkert et al. (1991), } \\
\text { Sivagnanam et al. (1990) }\end{array}$ \\
\hline bul_sc08_0190 & & & \\
\hline bul_sc08_0616 & & & \\
\hline bul_sc08_1081 & & & \\
\hline bul_sc08_1622 & & & \\
\hline bul_sc08_1687 & & & \\
\hline bul_sc08_1693 & & & \\
\hline bul_sc08_2299 & & & \\
\hline bul_sc08_2311 & & & \\
\hline bul_sc09_0078 & & & \\
\hline bul_sc09_0210 & & & \\
\hline bul_sc09_0286 & & & \\
\hline bul_sc09_0320 & & & \\
\hline bul_sc09_0321 & & & \\
\hline bul_sc09_0322 & & & \\
\hline bul_sc09_0414 & & & \\
\hline bul_sc09_0536 & & & \\
\hline bul_sc09_0539 & & & \\
\hline bul_sc09_0660 & & & \\
\hline bul_sc09_0689 & & & \\
\hline bul_sc09_1074 & & & \\
\hline bul_sc09_1113 & & & \\
\hline bul_sc09_1115 & & & \\
\hline bul_sc09_1177 & & & \\
\hline bul_sc09_1180 & & & \\
\hline bul_sc09_1233 & & & \\
\hline bul_sc09_1610 & & & \\
\hline bul_sc09_1767 & & & \\
\hline bul_sc09_1769 & & & \\
\hline bul_sc09_1771 & & & \\
\hline bul_sc10_0079 & & & \\
\hline bul_sc10_0256 & & & \\
\hline bul_sc10_0268 & & & \\
\hline bul_sc10_0318 & & & \\
\hline bul_sc10_0406 & & & \\
\hline
\end{tabular}


M. A. T. Groenewegen and J. A. D. L. Blommaert: Mira variables in the bulge, Online Material p 18

Table 2. continued.

\begin{tabular}{|c|c|c|c|}
\hline OGLE-name & Other name & Chemical type & Remarks \\
\hline bul_sc10_0792 & & & \\
\hline bul_sc10 0937 & & & \\
\hline bul_sc10_1238 & & & \\
\hline bul_sc10_1411 & & & \\
\hline bul_sc10_1456 & & & \\
\hline bul_sc10_1690 & & & \\
\hline bul_sc10_1979 & & & \\
\hline bul_sc10_2017 & & & \\
\hline bul_sc10_2048 & & & \\
\hline bul_sc10_2052 & & & \\
\hline bul_sc10_2383 & & & \\
\hline bul_sc11_0274 & & & \\
\hline bul_sc11_0416 & & & \\
\hline bul_sc11_0709 & & & \\
\hline bul_sc11_0795 & IRAS_18177-2233 & & \\
\hline bul_sc11_0850 & & & \\
\hline bul_sc11_0857 & & & \\
\hline bul_sc11_1132 & IRAS_18180-2225 & & \\
\hline bul_sc11_1432 & & & \\
\hline bul_sc11_1448 & & & \\
\hline bul_sc11_1528 & & & \\
\hline bul_sc11_1531 & & & \\
\hline bul_sc11_1532 & & & \\
\hline bul_sc11_1540 & & & \\
\hline bul_sc11_1743 & & & \\
\hline bul_sc11_1916 & & & \\
\hline bul_sc11_1930 & & & \\
\hline bul_sc11_2001 & & & \\
\hline bul_sc11_2049 & & & \\
\hline bul_sc12_0116 & & & \\
\hline bul_sc12_0124 & & & \\
\hline bul_sc12_0353 & & & \\
\hline bul_sc12_0412 & & & \\
\hline bul_sc12_0508 & & & \\
\hline bul_sc12_0509 & & & \\
\hline bul_sc12_0573 & & & \\
\hline bul_sc12_0700 & & & \\
\hline bul_sc12_0804 & IRAS_18131-2413 & & \\
\hline bul_sc12_0809 & & & \\
\hline bul_sc12_0827 & & & \\
\hline bul_sc12_0995 & & & \\
\hline bul_sc12_0999 & & & \\
\hline bul_sc12_1066 & & & \\
\hline bul_sc12_1428 & & & \\
\hline bul_sc12_2084 & & & \\
\hline bul_sc12_2115 & & & \\
\hline bul_sc12_2149 & & & \\
\hline bul_sc12_2267 & & & \\
\hline bul_sc12_2346 & & & \\
\hline bul_sc12_2375 & & & \\
\hline bul_sc12_2395 & & & \\
\hline bul_sc12_2403 & & & \\
\hline bul_sc12_2435 & & & \\
\hline bul_sc12_2454 & & & \\
\hline bul_sc12_2487 & & & \\
\hline bul_sc12_2502 & & & \\
\hline bul_sc12_2599 & IRAS_18125-2345 & & \\
\hline bul_sc12_2905 & & & \\
\hline bul_sc12_3025 & IRAS_18131-2338 & & \\
\hline
\end{tabular}


M. A. T. Groenewegen and J. A. D. L. Blommaert: Mira variables in the bulge, Online Material p 19

Table 2. continued.

\begin{tabular}{|c|c|c|c|}
\hline OGLE-name & Other name & Chemical type & Remarks \\
\hline bul_sc12_3370 & & & \\
\hline bul_sc12 3386 & & & \\
\hline bul_sc12_3387 & & & \\
\hline bul_sc13_0045 & & & \\
\hline bul_sc13_0151 & & & \\
\hline bul_sc13_0324 & & & \\
\hline bul_sc13_0605 & & & \\
\hline bul_sc13_0689 & & & \\
\hline bul_sc13_0909 & & & \\
\hline bul_sc13_1016 & & & \\
\hline bul_sc13_1142 & & & \\
\hline bul_sc13_1286 & & & \\
\hline bul_sc13_1542 & & & \\
\hline bul_sc13_1563 & & & \\
\hline bul_sc13_1581 & & & \\
\hline bul_sc13_1867 & & & \\
\hline bul_sc13_1986 & & & \\
\hline bul_sc13_2194 & & & \\
\hline bul_sc13_2245 & & & \\
\hline bul_sc13_2413 & & & \\
\hline bul_sc13_2662 & & & \\
\hline bul_sc13_2868 & & & \\
\hline bul_sc13_2987 & & & \\
\hline bul_sc13_3002 & & & \\
\hline bul_sc14_0057 & & & \\
\hline bul_sc14_0061 & & & \\
\hline bul_sc14_0104 & & & \\
\hline bul_sc14_0106 & & & \\
\hline bul_sc14_0189 & & & \\
\hline bul_sc14_0212 & & & \\
\hline bul_sc14_0359 & & & \\
\hline bul_sc14_0378 & & & \\
\hline bul_sc14_0582 & & & \\
\hline bul_sc14_0636 & & & \\
\hline bul_sc14_0650 & & & \\
\hline bul_sc14_0894 & & & \\
\hline bul_sc14_0900 & & & \\
\hline bul_sc14_0969 & & & \\
\hline bul_sc14_1172 & & & \\
\hline bul_sc14_1275 & & & \\
\hline bul_sc14_1349 & & & \\
\hline bul_sc14_1361 & & & \\
\hline bul_sc14_1616 & & & \\
\hline bul_sc14_1671 & & & \\
\hline bul_sc14_1673 & & & \\
\hline bul_sc14_1685 & & & \\
\hline bul_sc14_1810 & & & \\
\hline bul_sc14_1854 & & & \\
\hline bul_sc14_2081 & & & \\
\hline bul_sc14_2456 & & & \\
\hline bul_sc14_2459 & & & \\
\hline bul_sc14_2466 & & & \\
\hline bul_sc14_2527 & & & \\
\hline bul_sc14_2579 & & & \\
\hline bul_sc14_2664 & & & \\
\hline bul_sc14_2733 & & & \\
\hline bul_sc14_2913 & & & \\
\hline bul_sc14_2914 & & & \\
\hline bul_sc14_2921 & & & \\
\hline
\end{tabular}


M. A. T. Groenewegen and J. A. D. L. Blommaert: Mira variables in the bulge, Online Material p 20

Table 2. continued.

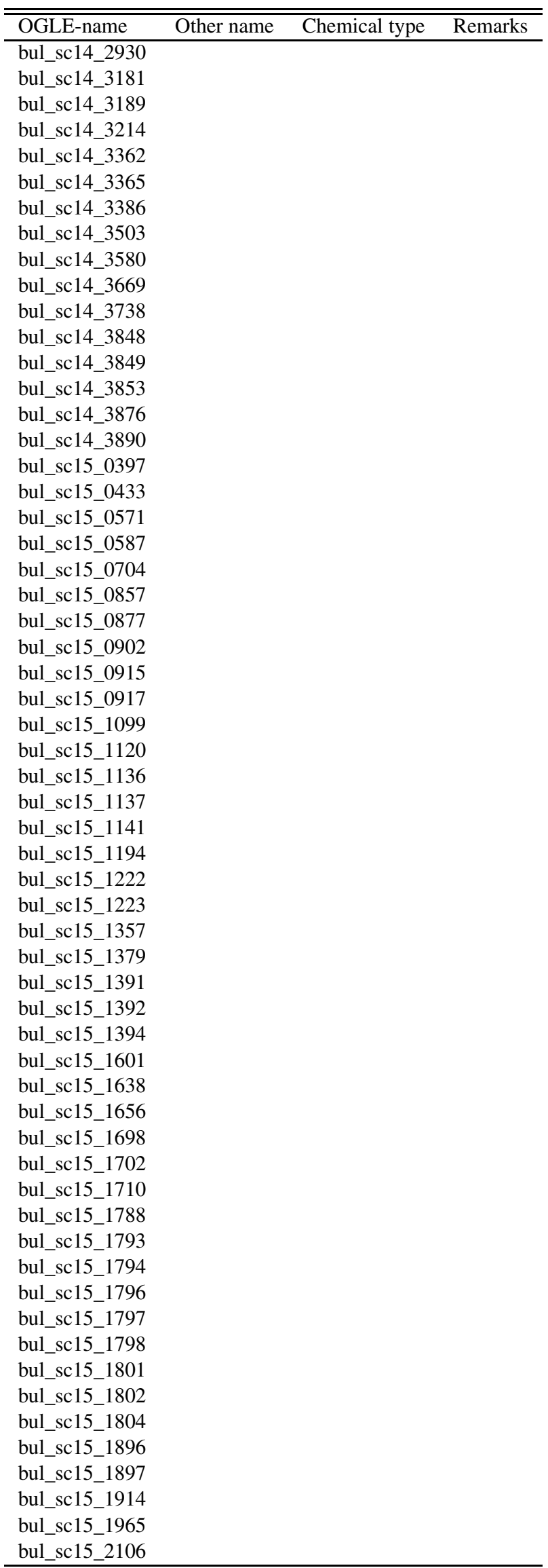


M. A. T. Groenewegen and J. A. D. L. Blommaert: Mira variables in the bulge, Online Material p 21

Table 2. continued.

\begin{tabular}{|c|c|c|c|}
\hline OGLE-name & Other name & Chemical type & Remarks \\
\hline bul_sc15_2111 & & & \\
\hline bul_sc15_2135 & & & \\
\hline bul_sc15_2170 & & & \\
\hline bul_sc15_2182 & & & \\
\hline bul_sc15_2304 & & & \\
\hline bul_sc15_2314 & & & \\
\hline bul_sc15_2360 & & & \\
\hline bul_sc15_2511 & & & \\
\hline bul_sc15_2722 & & & \\
\hline bul_sc15_2794 & & & \\
\hline bul_sc15_2869 & & & \\
\hline bul_sc15_3075 & & & \\
\hline bul_sc15_3171 & & & \\
\hline bul_sc15_3189 & & & \\
\hline bul_sc15_3190 & & & \\
\hline bul_sc15_3244 & & & \\
\hline bul_sc15_3254 & & & \\
\hline bul_sc15_3360 & & & \\
\hline bul_sc15_3401 & & & \\
\hline bul_sc15_3445 & & & \\
\hline bul_sc15_3452 & & & \\
\hline bul_sc15_3530 & & & \\
\hline bul_sc15_3539 & & & \\
\hline bul_sc15_3540 & & & \\
\hline bul_sc15_3599 & & & \\
\hline bul_sc15_3611 & & & \\
\hline bul_sc15_3760 & & & \\
\hline bul_sc15_3771 & & & \\
\hline bul_sc16_0211 & & & \\
\hline bul_sc16_0280 & & & \\
\hline bul_sc16_0645 & & & \\
\hline bul_sc16_0653 & & & \\
\hline bul_sc16_0663 & & & \\
\hline bul_sc16_0804 & & & \\
\hline bul_sc16_0926 & & & \\
\hline bul_sc16_0968 & & & \\
\hline bul_sc16_1060 & & & \\
\hline bul_sc16_1428 & & & \\
\hline bul_sc16_1777 & & & \\
\hline bul_sc16_1822 & & & \\
\hline bul_sc16_2046 & & & \\
\hline bul_sc16_2164 & & & \\
\hline bul_sc16_2494 & & & \\
\hline bul_sc16_2496 & & & \\
\hline bul_sc16_2841 & & & \\
\hline bul_sc16_3224 & & & \\
\hline bul_sc16_3270 & & & \\
\hline bul_sc16_3572 & & & \\
\hline bul_sc16_3608 & & & \\
\hline bul_sc16_3667 & & & \\
\hline bul_sc16_3693 & & & \\
\hline bul_sc16_3699 & & & \\
\hline bul_sc16_3700 & & & \\
\hline bul_sc16_3701 & & & \\
\hline bul_sc16_3819 & & & \\
\hline bul_sc16_3857 & IRAS_18065-2601 & & \\
\hline bul_sc16_3861 & & & \\
\hline bul_sc16_4025 & & & \\
\hline bul_sc16_4082 & & & \\
\hline
\end{tabular}


M. A. T. Groenewegen and J. A. D. L. Blommaert: Mira variables in the bulge, Online Material p 22

Table 2. continued.

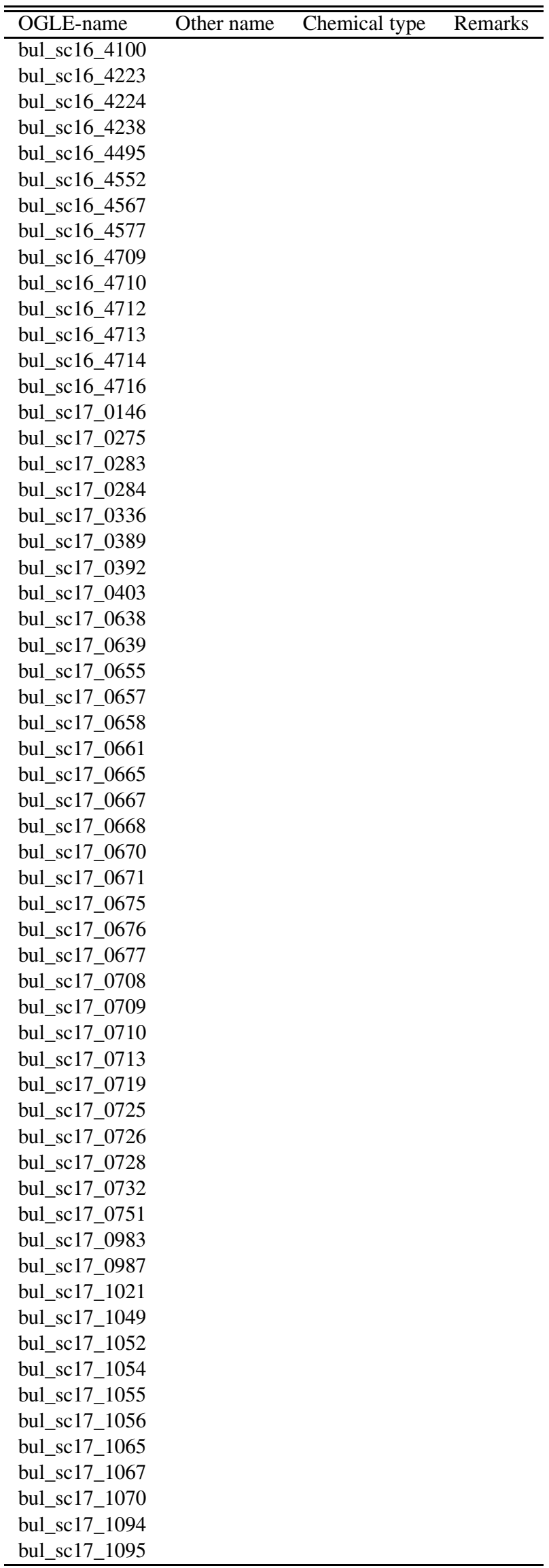


M. A. T. Groenewegen and J. A. D. L. Blommaert: Mira variables in the bulge, Online Material p 23

Table 2. continued.

\begin{tabular}{|c|c|c|c|}
\hline OGLE-name & Other name & Chemical type & $\overline{\text { Remarks }}$ \\
\hline bul_sc17_1101 & & & \\
\hline bul_sc17_1157 & & & \\
\hline bul_sc17_1353 & & & \\
\hline bul_sc17_1366 & & & \\
\hline bul_sc17_1368 & & & \\
\hline bul_sc17_1369 & & & \\
\hline bul_sc17_1372 & & & \\
\hline bul_sc17_1378 & & & \\
\hline bul_sc17_1504 & IRAS_18079-2623 & & \\
\hline bul_sc17_1583 & & & \\
\hline bul_sc17_1595 & & & \\
\hline bul_sc17_1601 & & & \\
\hline bul_sc17_1602 & & & \\
\hline bul_sc17_1607 & & & \\
\hline bul_sc17_1608 & & & \\
\hline bul_sc17_1612 & & & \\
\hline bul_sc17_1613 & & & \\
\hline bul_sc17_1614 & & & \\
\hline bul_sc17_1616 & & & \\
\hline bul_sc17_1633 & & & \\
\hline bul_sc17_1644 & & & \\
\hline bul_sc17_1659 & & & \\
\hline bul_sc17_1699 & & & \\
\hline bul_sc17_1783 & & & \\
\hline bul_sc17_1795 & & & \\
\hline bul_sc17_1797 & & & \\
\hline bul_sc17_1803 & & & \\
\hline bul_sc17_1953 & & & \\
\hline bul_sc17_1964 & & & \\
\hline bul_sc17_2116 & & & \\
\hline bul_sc17_2129 & & & \\
\hline bul_sc17_2292 & & & \\
\hline bul_sc17_2296 & & & \\
\hline bul_sc17_2297 & & & \\
\hline bul_sc17_2298 & & & \\
\hline bul_sc17_2301 & & & \\
\hline bul_sc17_2302 & & & \\
\hline bul_sc17_2303 & & & \\
\hline bul_sc17_2304 & & & \\
\hline bul_sc17_2305 & & & \\
\hline bul_sc17_2306 & & & \\
\hline bul_sc17_2308 & & & \\
\hline bul_sc17_2309 & & & \\
\hline bul_sc17_2311 & & & \\
\hline bul_sc17_2312 & & & \\
\hline bul_sc17_2314 & & & \\
\hline bul_sc17_2316 & & & \\
\hline bul_sc17_2317 & & & \\
\hline bul_sc17_2318 & & & \\
\hline bul_sc17_2319 & & & \\
\hline bul_sc17_2320 & & & \\
\hline bul_sc17_2321 & & & \\
\hline bul_sc17_2322 & & & \\
\hline bul_sc17_2323 & & & \\
\hline bul_sc17_2324 & & & \\
\hline bul_sc17_2493 & & & \\
\hline bul_sc17_2552 & & & \\
\hline bul_sc17_2565 & & & \\
\hline bul_sc17_2566 & & & \\
\hline
\end{tabular}


M. A. T. Groenewegen and J. A. D. L. Blommaert: Mira variables in the bulge, Online Material p 24

Table 2. continued.

\begin{tabular}{|c|c|c|c|}
\hline OGLE-name & Other name & Chemical type & $\overline{\text { Remarks }}$ \\
\hline bul_sc17_2568 & & & \\
\hline bul_sc17_2578 & & & \\
\hline bul_sc17_2579 & & & \\
\hline bul_sc17_2580 & & & \\
\hline bul_sc17_2704 & & & \\
\hline bul_sc17_2867 & & & \\
\hline bul_sc17_2949 & & & \\
\hline bul_sc17_2953 & & & \\
\hline bul_sc17_2957 & & & \\
\hline bul_sc17_2958 & & & \\
\hline bul_sc17_2960 & & & \\
\hline bul_sc17_2961 & & & \\
\hline bul_sc17_2964 & & & \\
\hline bul_sc17_2966 & & & \\
\hline bul_sc17_2967 & & & \\
\hline bul_sc17_2969 & & & \\
\hline bul_sc17_3009 & & & \\
\hline bul_sc17_3019 & & & \\
\hline bul_sc17_3191 & & & \\
\hline bul_sc17_3199 & & & \\
\hline bul_sc17_3200 & & & \\
\hline bul_sc17_3210 & & & \\
\hline bul_sc17_3216 & & & \\
\hline bul_sc17_3218 & & & \\
\hline bul_sc17_3219 & & & \\
\hline bul_sc17_3253 & & & \\
\hline bul_sc17_3266 & & & \\
\hline bul_sc17_3269 & & & \\
\hline bul_sc17_3277 & MM7-B_V98_EB & & \\
\hline bul_sc17_3280 & & & \\
\hline bul_sc17_3281 & & & \\
\hline bul_sc17_3350 & & & \\
\hline bul_sc17_3434 & & & \\
\hline bul_sc17_3437 & & & \\
\hline bul_sc17_3492 & IRAS_18075-2558 & & \\
\hline bul_sc17_4011 & & & \\
\hline bul_sc17_4016 & & & \\
\hline bul_sc17_4017 & & & \\
\hline bul_sc17_4019 & & & \\
\hline bul_sc17_4020 & & & \\
\hline bul_sc17_4021 & & & \\
\hline bul_sc17_4062 & & & \\
\hline bul_sc17_4063 & & & \\
\hline bul_sc17_4065 & & & \\
\hline bul_sc17_4067 & & & \\
\hline bul_sc17_4068 & & & \\
\hline bul_sc17_4092 & & & \\
\hline bul_sc17_4100 & & & \\
\hline bul_sc17_4116 & & & \\
\hline bul_sc17_4127 & & & \\
\hline bul_sc17_4131 & & & \\
\hline bul_sc17_4133 & & & \\
\hline bul_sc17_4163 & & & \\
\hline bul_sc17_4166 & & & \\
\hline bul_sc17_4168 & & & \\
\hline bul_sc17_4217 & & & \\
\hline bul_sc17_4221 & & & \\
\hline bul_sc17_4222 & & & \\
\hline
\end{tabular}


M. A. T. Groenewegen and J. A. D. L. Blommaert: Mira variables in the bulge, Online Material p 25

Table 2. continued.

\begin{tabular}{|c|c|c|c|}
\hline OGLE-name & Other name & Chemical type & Remarks \\
\hline bul_sc17_4226 & & & \\
\hline bul_sc17 4271 & & & \\
\hline bul_sc17_4282 & & & \\
\hline bul_sc17_4358 & & & \\
\hline bul_sc17_4516 & & & \\
\hline bul_sc18_0202 & & & \\
\hline bul_sc18_0471 & & & \\
\hline bul_sc18_0472 & & & \\
\hline bul_sc18_0698 & & & \\
\hline bul_sc18_0779 & & & \\
\hline bul_sc18_0788 & & & \\
\hline bul_sc18_0835 & & & \\
\hline bul_sc18_0883 & & & \\
\hline bul_sc18_0884 & & & \\
\hline bul_sc18_0900 & & & \\
\hline bul_sc18_0903 & & & \\
\hline bul_sc18_0905 & & & \\
\hline bul_sc18_0910 & & & \\
\hline bul_sc18_0981 & & & \\
\hline bul_sc18_0995 & & & \\
\hline bul_sc18_1050 & & & \\
\hline bul_sc18_1065 & & & \\
\hline bul_sc18_1066 & & & \\
\hline bul_sc18_1140 & & & \\
\hline bul_sc18_1381 & & & \\
\hline bul_sc18_1485 & & & \\
\hline bul_sc18_1486 & & & \\
\hline bul_sc18_1588 & & & \\
\hline bul_sc18_1597 & & & \\
\hline bul_sc18_1600 & & & \\
\hline bul_sc18_1617 & & & \\
\hline bul_sc18_1821 & & & \\
\hline bul_sc18_1832 & & & \\
\hline bul_sc18_1925 & & & \\
\hline bul_sc18_2067 & & & \\
\hline bul_sc18_2240 & & & \\
\hline bul_sc18_2631 & & & \\
\hline bul_sc18_2652 & & & \\
\hline bul_sc18_2665 & & & \\
\hline bul_sc18_3398 & & & \\
\hline bul_sc18_3509 & & & \\
\hline bul_sc18_3592 & & & \\
\hline bul_sc18_3630 & & & \\
\hline bul_sc18_3654 & & & \\
\hline bul_sc18_3951 & & & \\
\hline bul_sc18_4066 & & & \\
\hline bul_sc18_4202 & & & \\
\hline bul_sc18_4640 & & & \\
\hline bul_sc18_4666 & & & \\
\hline bul_sc18_4688 & & & \\
\hline bul_sc18_4703 & & & \\
\hline bul_sc18_4794 & & & \\
\hline bul_sc18_4809 & & & \\
\hline bul_sc18_4914 & & & \\
\hline bul_sc18_4923 & & & \\
\hline bul_sc18_5001 & & & \\
\hline bul_sc18_5342 & & & \\
\hline bul_sc18_5361 & & & \\
\hline bul_sc18_5379 & IRAS_18039-2649 & & \\
\hline
\end{tabular}


M. A. T. Groenewegen and J. A. D. L. Blommaert: Mira variables in the bulge, Online Material p 26

Table 2. continued.

\begin{tabular}{|c|c|c|c|}
\hline OGLE-name & Other name & Chemical type & Remarks \\
\hline \multicolumn{4}{|l|}{ bul_sc18_5569 } \\
\hline \multicolumn{4}{|l|}{ bul_sc19_0505 } \\
\hline \multicolumn{4}{|l|}{ bul_sc19_0506 } \\
\hline \multicolumn{4}{|l|}{ bul_sc19_0608 } \\
\hline \multicolumn{4}{|l|}{ bul_sc19_0655 } \\
\hline \multicolumn{4}{|l|}{ bul_sc19_0664 } \\
\hline \multicolumn{4}{|l|}{ bul_sc19_0734 } \\
\hline \multicolumn{4}{|l|}{ bul_sc19_0747 } \\
\hline \multicolumn{4}{|l|}{ bul_sc19_0760 } \\
\hline \multicolumn{4}{|l|}{ bul_sc19_0867 } \\
\hline \multicolumn{4}{|l|}{ bul_sc19_0890 } \\
\hline \multicolumn{4}{|l|}{ bul_sc19_0891 } \\
\hline \multicolumn{4}{|l|}{ bul_sc19_0892 } \\
\hline bul_sc19_0961 & IRAS_18046-2730 & & \\
\hline \multicolumn{4}{|l|}{ bul_sc19_1373 } \\
\hline \multicolumn{4}{|l|}{ bul_sc19_1394 } \\
\hline \multicolumn{4}{|l|}{ bul_sc19_1402 } \\
\hline \multicolumn{4}{|l|}{ bul_sc19_1436 } \\
\hline \multicolumn{4}{|l|}{ bul_sc19_1587 } \\
\hline bul_sc19_1614 & & & \\
\hline bul_sc19_1621 & & & \\
\hline bul_sc19_2131 & & & \\
\hline bul_sc19_2139 & & & \\
\hline bul_sc19_2302 & & & \\
\hline bul_sc19_2330 & & & \\
\hline bul_sc19_2332 & V 4720 Sgr & & \\
\hline bul_sc19_2449 & & & \\
\hline bul_sc19_2462 & & & \\
\hline bul_sc19_2486 & & & \\
\hline bul_sc19_2948 & & & \\
\hline bul_sc19_2950 & & & \\
\hline bul_sc19_3196 & & & \\
\hline bul_sc19_3310 & & & \\
\hline bul_sc19_3334 & & & \\
\hline bul_sc19_3335 & & & \\
\hline bul_sc19_3487 & & & \\
\hline bul_sc19_3581 & & & \\
\hline bul_sc19_3641 & & & \\
\hline bul_sc19_3787 & & & \\
\hline bul_sc19_3797 & & & \\
\hline bul_sc19_3831 & IRAS_18050-2659 & & $\begin{array}{l}\text { Kwok et al. (1997), Izumiura et al. (1995), } \\
\text { te Lintel Hekkert (1991) }\end{array}$ \\
\hline bul_sc19_4048 & & & \\
\hline bul_sc19_4066 & & & \\
\hline bul_sc19_4132 & & & \\
\hline bul_sc19_4344 & & & \\
\hline bul_sc19_4423 & & & \\
\hline bul_sc19_4430 & & & \\
\hline bul_sc19_4649 & & & \\
\hline bul_sc19_4777 & & & \\
\hline bul_sc19_4831 & & & \\
\hline bul_sc19_4879 & & & \\
\hline bul_sc19_4898 & & & \\
\hline bul_sc20_0147 & & & \\
\hline bul_sc20_0150 & & & \\
\hline bul_sc20_0170 & & & \\
\hline bul_sc20_0190 & & & \\
\hline bul_sc20_0197 & & & \\
\hline
\end{tabular}


Table 2. continued.

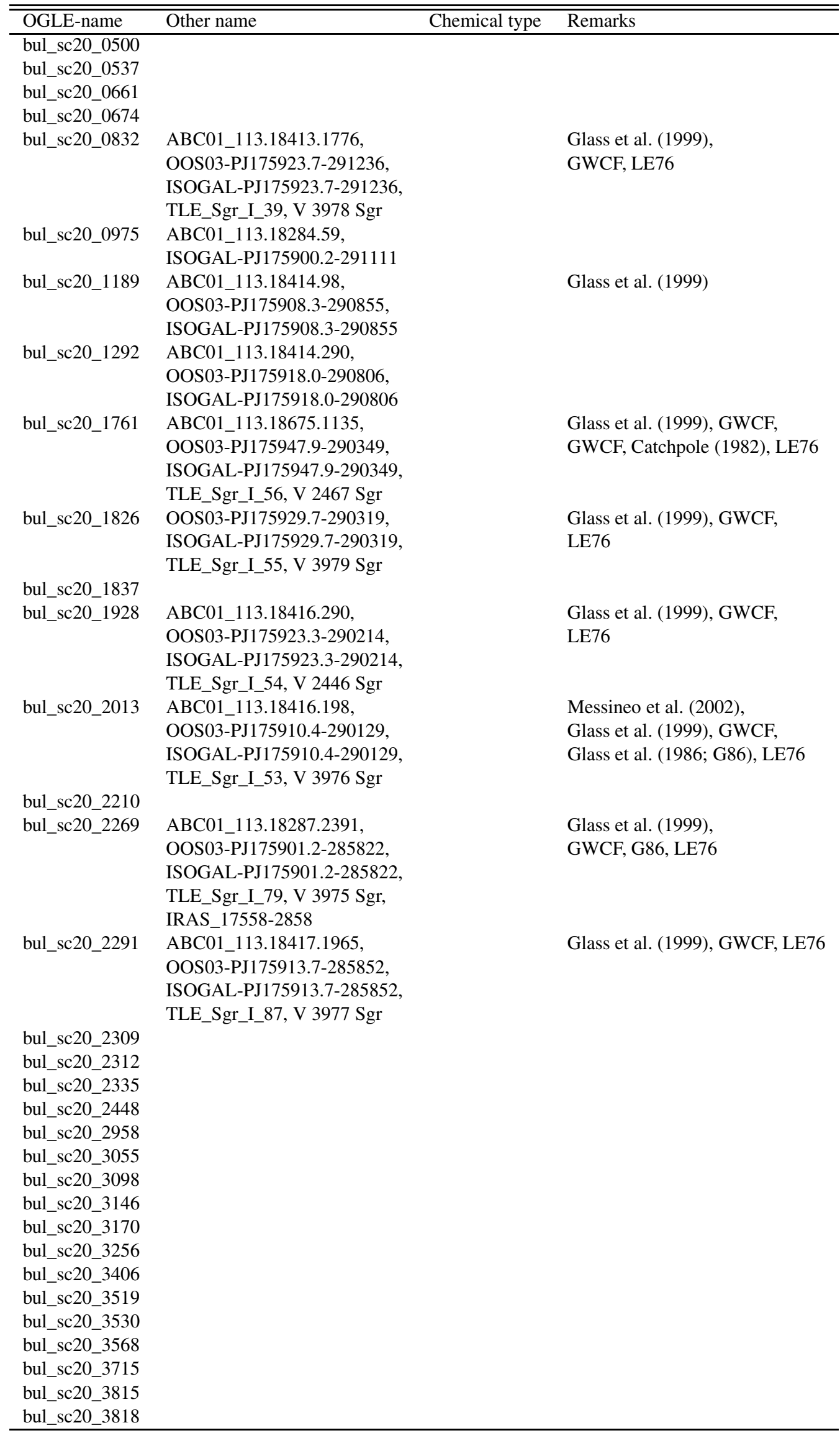


M. A. T. Groenewegen and J. A. D. L. Blommaert: Mira variables in the bulge, Online Material p 28

Table 2. continued.

\begin{tabular}{|c|c|c|c|}
\hline OGLE-name & Other name & Chemical type & $\overline{\text { Remarks }}$ \\
\hline bul_sc20_3886 & & & \\
\hline bul_sc20_3891 & & & \\
\hline bul_sc20_4367 & & & \\
\hline bul_sc20_4404 & & & \\
\hline bul_sc20_4413 & & & \\
\hline bul_sc20_4621 & & & \\
\hline bul_sc20_4675 & & & \\
\hline bul_sc20_4763 & & & \\
\hline bul_sc20_4768 & & & \\
\hline bul_sc20_4790 & & & \\
\hline bul_sc20_4874 & & & \\
\hline bul_sc20_4939 & & & \\
\hline bul_sc20_4954 & & & \\
\hline bul_sc20_5108 & & & \\
\hline bul_sc20_5141 & & & \\
\hline bul_sc20_5147 & & & \\
\hline bul_sc20_5152 & & & \\
\hline bul_sc20_5158 & & & \\
\hline bul_sc20_5159 & & & \\
\hline bul_sc20_5171 & & & \\
\hline bul_sc20_5315 & & & \\
\hline bul_sc20_5369 & & & \\
\hline bul_sc20_5657 & & & \\
\hline bul_sc20_5736 & & & \\
\hline bul_sc20_5781 & & & \\
\hline bul_sc20_5840 & & & \\
\hline bul_sc21_0035 & & & \\
\hline bul_sc21_0055 & & & \\
\hline bul_sc21_0209 & & & \\
\hline bul_sc21_0531 & & & \\
\hline bul_sc21_0635 & & & \\
\hline bul_sc21_0740 & & & \\
\hline bul_sc21_1228 & & & \\
\hline bul_sc21_1235 & & & \\
\hline bul_sc21_1258 & & & \\
\hline bul_sc21_1415 & & & \\
\hline bul_sc21_1433 & & & \\
\hline bul_sc21_1459 & & & \\
\hline bul_sc21_1463 & & & \\
\hline bul_sc21_1615 & & & \\
\hline bul_sc21_1745 & & & \\
\hline bul_sc21_1954 & & & \\
\hline bul_sc21_1982 & & & \\
\hline bul_sc21_2008 & & & \\
\hline bul_sc21_2679 & & & \\
\hline bul_sc21_2895 & & & \\
\hline bul_sc21_2919 & & & \\
\hline bul_sc21_3198 & & & \\
\hline bul_sc21_3216 & & & \\
\hline bul_sc21_3239 & & & \\
\hline bul_sc21_3519 & & & \\
\hline bul_sc21_3541 & & & \\
\hline bul_sc21_3611 & & & \\
\hline bul_sc21_3629 & & & \\
\hline bul_sc21_3954 & & & \\
\hline bul_sc21_4176 & & & \\
\hline bul_sc21_4627 & & & \\
\hline bul_sc21_4690 & & & \\
\hline bul_sc21_4849 & & & \\
\hline
\end{tabular}


M. A. T. Groenewegen and J. A. D. L. Blommaert: Mira variables in the bulge, Online Material p 29

Table 2. continued.

\begin{tabular}{|c|c|c|c|}
\hline OGLE-name & Other name & Chemical type & $\overline{\text { Remarks }}$ \\
\hline bul_sc21_4940 & & & \\
\hline bul_sc21_5109 & & & \\
\hline bul $\mathrm{sc} 215112$ & & & \\
\hline bul_sc21_5129 & & & \\
\hline bul_sc21_5301 & & & \\
\hline bul_sc21_5358 & & & \\
\hline bul_sc21_5569 & & & \\
\hline bul_sc21_5635 & & & \\
\hline bul_sc21_5654 & & & \\
\hline bul_sc21_5727 & & & \\
\hline bul_sc21_6155 & & & \\
\hline bul_sc21_6187 & & & \\
\hline bul_sc21_6221 & & & \\
\hline bul_sc21_6291 & & & \\
\hline bul_sc21_6346 & & & \\
\hline bul_sc21_6427 & & & \\
\hline bul_sc21_6462 & & & \\
\hline bul_sc21_6542 & & & \\
\hline bul_sc21_6564 & & & \\
\hline bul_sc21_6647 & & & \\
\hline bul_sc21_6683 & & & \\
\hline bul_sc21_6792 & & & \\
\hline bul_sc21_7104 & & & \\
\hline bul_sc21_7140 & & & \\
\hline bul_sc21_7167 & & & \\
\hline bul_sc21_7392 & & & \\
\hline bul_sc21_7403 & & & \\
\hline bul_sc22_0158 & & & \\
\hline bul_sc22_0372 & IRAS_17531-3110 & & \\
\hline bul_sc22_0384 & & & \\
\hline bul_sc22_0434 & & & \\
\hline bul_sc22_0689 & & & \\
\hline bul_sc22_0693 & & & \\
\hline bul_sc22_0698 & & & \\
\hline bul_sc22_0711 & & & \\
\hline bul_sc22_0771 & & & \\
\hline bul_sc22_0986 & & & \\
\hline bul_sc22_1083 & & & \\
\hline bul_sc22_1086 & & & \\
\hline bul_sc22_1150 & & & \\
\hline bul_sc22_1230 & & & \\
\hline bul_sc22_1319 & & & \\
\hline bul_sc22_1382 & & & \\
\hline bul_sc22_1395 & & & \\
\hline bul_sc22_1579 & & & \\
\hline bul_sc22_1599 & & & \\
\hline bul_sc22_1726 & & & \\
\hline bul_sc22_1841 & & & \\
\hline bul_sc22_1984 & & & \\
\hline bul_sc22_1997 & & & \\
\hline bul_sc22_2093 & & & \\
\hline bul_sc22_2269 & & & \\
\hline bul_sc22_2331 & IRAS_17540-3053 & & \\
\hline bul_sc22_2351 & & & \\
\hline bul_sc22_2354 & & & \\
\hline bul_sc22_2395 & & & \\
\hline bul_sc22_2403 & & & \\
\hline bul_sc22_2407 & & & \\
\hline bul_sc22_2471 & & & \\
\hline
\end{tabular}


M. A. T. Groenewegen and J. A. D. L. Blommaert: Mira variables in the bulge, Online Material p 30

Table 2. continued.

\begin{tabular}{|c|c|c|c|}
\hline \multirow{2}{*}{\multicolumn{4}{|c|}{$\frac{\text { OGLE-name }}{\text { bul_sc22_2678 }}$}} \\
\hline & & & \\
\hline \multicolumn{4}{|l|}{ bul_sc22_2790 } \\
\hline \multicolumn{4}{|l|}{ bul_sc22_2989 } \\
\hline \multicolumn{4}{|l|}{ bul_sc22_3006 } \\
\hline \multicolumn{4}{|l|}{ bul_sc22_3025 } \\
\hline \multicolumn{4}{|l|}{ bul_sc22_3036 } \\
\hline \multicolumn{4}{|l|}{ bul_sc22_3143 } \\
\hline \multicolumn{4}{|l|}{ bul_sc22_3184 } \\
\hline \multicolumn{4}{|l|}{ bul_sc22_3293 } \\
\hline \multicolumn{4}{|l|}{ bul_sc22_3304 } \\
\hline \multicolumn{4}{|l|}{ bul_sc22_3440 } \\
\hline \multicolumn{4}{|l|}{ bul_sc22_3512 } \\
\hline \multicolumn{4}{|l|}{ bul_sc22_3535 } \\
\hline \multicolumn{4}{|l|}{ bul_sc22_3810 } \\
\hline \multicolumn{4}{|l|}{ bul_sc22_4005 } \\
\hline \multicolumn{4}{|l|}{ bul_sc22_4177 } \\
\hline \multicolumn{4}{|l|}{ bul_sc22_4224 } \\
\hline bul_sc22_4288 & & & \\
\hline bul_sc22_4296 & & & \\
\hline bul_sc22_4414 & & & \\
\hline bul_sc22_4618 & & & \\
\hline bul_sc22_4619 & & & \\
\hline bul_sc22_4621 & & & \\
\hline bul_sc22_4717 & & & \\
\hline bul_sc22_4731 & & & \\
\hline bul_sc22_4744 & & & \\
\hline bul_sc22_4765 & & & \\
\hline bul_sc22_4866 & & & \\
\hline bul_sc22_4905 & & & \\
\hline bul_sc22_4908 & & & \\
\hline bul_sc22_4993 & & & \\
\hline bul_sc22_5238 & & & \\
\hline bul_sc22_5239 & & & \\
\hline bul_sc22_5309 & & & \\
\hline bul_sc22_5512 & & & \\
\hline bul_sc22_5515 & & & \\
\hline bul_sc22_5562 & & & \\
\hline bul_sc22_5571 & & & \\
\hline bul_sc23_0073 & & & \\
\hline bul_sc23_0133 & & & \\
\hline bul_sc23_0134 & & & \\
\hline bul_sc23_0189 & & & \\
\hline bul_sc23_0200 & & & \\
\hline bul_sc23_0414 & & & \\
\hline bul_sc23_0432 & & & \\
\hline bul_sc23_0661 & IRAS_17545-3132 & & \\
\hline bul_sc23_0723 & & & \\
\hline bul_sc23_0933 & & & \\
\hline bul_sc23_0963 & & & \\
\hline bul_sc23_0965 & & & \\
\hline bul_sc23_1037 & & & \\
\hline bul_sc23_1095 & & & \\
\hline bul_sc23_1114 & & & \\
\hline bul_sc23_1148 & & & \\
\hline bul_sc23_1188 & & & \\
\hline bul_sc23_1362 & & & \\
\hline bul_sc23_1372 & & & \\
\hline bul_sc23_1376 & & & \\
\hline bul_sc23_1612 & & & \\
\hline
\end{tabular}


M. A. T. Groenewegen and J. A. D. L. Blommaert: Mira variables in the bulge, Online Material p 31

Table 2. continued.

\begin{tabular}{|c|c|c|c|}
\hline OGLE-name & Other name & Chemical type & $\overline{\text { Remarks }}$ \\
\hline bul_sc23_1821 & & & \\
\hline bul_sc23_1875 & & & \\
\hline bul sc23 1944 & & & \\
\hline bul_sc23_2209 & & & \\
\hline bul_sc23_2324 & & & \\
\hline bul_sc23_2351 & & & \\
\hline bul_sc23_2512 & & & \\
\hline bul_sc23_2513 & & & \\
\hline bul_sc23_2542 & & & \\
\hline bul_sc23_2545 & & & \\
\hline bul_sc23_2549 & & & \\
\hline bul_sc23_2641 & & & \\
\hline bul_sc23_2808 & & & \\
\hline bul_sc23_2837 & & & \\
\hline bul_sc23_2927 & & & \\
\hline bul_sc23_2952 & & & \\
\hline bul_sc23_3001 & & & \\
\hline bul_sc23_3145 & & & \\
\hline bul_sc23_3367 & & & \\
\hline bul_sc23_3368 & & & \\
\hline bul_sc23_3449 & & & \\
\hline bul_sc23_3467 & & & \\
\hline bul_sc23_3518 & & & \\
\hline bul_sc23_3598 & & & \\
\hline bul_sc23_3616 & & & \\
\hline bul_sc23_3628 & & & \\
\hline bul_sc23_3641 & & & \\
\hline bul_sc23_3678 & & & \\
\hline bul_sc23_3695 & & & \\
\hline bul_sc23_3711 & & & \\
\hline bul_sc23_3824 & & & \\
\hline bul_sc23_3870 & & & \\
\hline bul_sc23_3873 & & & \\
\hline bul_sc23_3916 & & & \\
\hline bul_sc23_4423 & & & \\
\hline bul_sc23_4585 & & & \\
\hline bul_sc23_4647 & & & \\
\hline bul_sc23_4766 & & & \\
\hline bul_sc23_4772 & & & \\
\hline bul_sc24_0090 & & & \\
\hline bul_sc24_0226 & & & \\
\hline bul_sc24_0296 & IRAS_17503-3315 & & \\
\hline bul_sc24_0311 & & & \\
\hline bul_sc24_0323 & & & \\
\hline bul_sc24_0324 & & & \\
\hline bul_sc24_0352 & & & \\
\hline bul_sc24_0394 & & & \\
\hline bul_sc24_0399 & & & \\
\hline bul_sc24_0412 & & & \\
\hline bul_sc24_0565 & & & \\
\hline bul_sc24_0668 & & & \\
\hline bul_sc24_0773 & & & \\
\hline bul_sc24_0835 & & & \\
\hline bul_sc24_0849 & & & \\
\hline bul_sc24_0850 & & & \\
\hline bul_sc24_0949 & & & \\
\hline bul_sc24_0989 & & & \\
\hline bul_sc24_1001 & & & \\
\hline bul_sc24_1012 & & & \\
\hline
\end{tabular}


M. A. T. Groenewegen and J. A. D. L. Blommaert: Mira variables in the bulge, Online Material p 32

Table 2. continued.

\begin{tabular}{|c|c|c|c|}
\hline OGLE-name & Other name & Chemical type & "Remarks \\
\hline bul_sc24_1065 & & & \\
\hline bul_sc24_1202 & & & \\
\hline bul_sc24_1203 & & & \\
\hline bul_sc24_1224 & & & \\
\hline bul_sc24_1230 & & & \\
\hline bul_sc24_1299 & & & \\
\hline bul_sc24_1580 & & & \\
\hline bul_sc24_1684 & & & \\
\hline bul_sc24_1765 & & & \\
\hline bul_sc24_1855 & & & \\
\hline bul_sc24_1915 & & & \\
\hline bul_sc24_2087 & & & \\
\hline bul_sc24_2106 & IRAS_17500-3250 & & \\
\hline bul_sc24_2129 & & & \\
\hline bul_sc24_2347 & & & \\
\hline bul_sc24_2421 & & & \\
\hline bul_sc24_2526 & & & \\
\hline bul_sc24_2595 & & & \\
\hline bul_sc24_2610 & & & \\
\hline bul_sc24_2744 & & & \\
\hline bul_sc24_2822 & & & \\
\hline bul_sc24_3033 & & & \\
\hline bul_sc24_3323 & & & \\
\hline bul_sc24_3399 & & & \\
\hline bul_sc24_3455 & & & \\
\hline bul_sc24_3456 & & & \\
\hline bul_sc24_3537 & & & \\
\hline bul_sc24_3547 & & & \\
\hline bul_sc24_3649 & & & \\
\hline bul_sc24_3665 & & & \\
\hline bul_sc24_3768 & & & \\
\hline bul_sc24_3820 & & & \\
\hline bul_sc24_4012 & & & \\
\hline bul_sc24_4119 & & & \\
\hline bul_sc24_4126 & & & \\
\hline bul_sc24_4273 & & & \\
\hline bul_sc25_0010 & IRAS_17511-3320 & & \\
\hline bul_sc25_0089 & & & \\
\hline bul_sc25_0093 & & & \\
\hline bul_sc25_0112 & & & \\
\hline bul_sc25_0125 & & & \\
\hline bul_sc25_0218 & & & \\
\hline bul_sc25_0341 & & & \\
\hline bul_sc25_0404 & & & \\
\hline bul_sc25_0409 & & & \\
\hline bul_sc25_0412 & & & \\
\hline bul_sc25_0413 & & & \\
\hline bul_sc25_0421 & & & \\
\hline bul_sc25_0459 & & & \\
\hline bul_sc25_0492 & & & \\
\hline bul_sc25_0527 & & & \\
\hline bul_sc25_0550 & & & \\
\hline bul_sc25_0559 & & & \\
\hline bul_sc25_0561 & & & \\
\hline bul_sc25_0612 & & & \\
\hline bul_sc25_0695 & & & \\
\hline bul_sc25_0733 & & & \\
\hline bul_sc25_0734 & & & \\
\hline bul_sc25_0971 & & & \\
\hline
\end{tabular}


M. A. T. Groenewegen and J. A. D. L. Blommaert: Mira variables in the bulge, Online Material p 33

Table 2. continued.

\begin{tabular}{|c|c|c|c|}
\hline OGLE-name & Other name & Chemical type & Remarks \\
\hline bul_sc25_1030 & & & \\
\hline bul_sc $25 \quad 1238$ & & & \\
\hline bul_sc25_1241 & & & \\
\hline bul_sc25_1276 & & & \\
\hline bul_sc25_1502 & & & \\
\hline bul_sc25_1505 & & & \\
\hline bul_sc25_1507 & & & \\
\hline bul_sc25_1557 & & & \\
\hline bul_sc25_1686 & & & \\
\hline bul_sc25_1771 & & & \\
\hline bul_sc25_1839 & & & \\
\hline bul_sc25_1851 & & & \\
\hline bul_sc25_1852 & & & \\
\hline bul_sc25_1909 & & & \\
\hline bul_sc25_1916 & & & \\
\hline bul_sc25_1917 & & & \\
\hline bul_sc25_1962 & & & \\
\hline bul_sc25_1969 & & & \\
\hline bul_sc25_1974 & & & \\
\hline bul_sc25_2019 & & & \\
\hline bul_sc25_2051 & & & \\
\hline bul_sc25_2053 & & & \\
\hline bul_sc25_2059 & & & \\
\hline bul_sc25_2162 & & & \\
\hline bul_sc25_2219 & & & \\
\hline bul_sc25_2472 & & & \\
\hline bul_sc25_2490 & & & \\
\hline bul_sc25_2499 & & & \\
\hline bul_sc25_2508 & & & \\
\hline bul_sc25_2555 & & & \\
\hline bul_sc25_2658 & & & \\
\hline bul_sc25_2659 & & & \\
\hline bul_sc25_2661 & & & \\
\hline bul_sc25_2667 & & & \\
\hline bul_sc25_2679 & & & \\
\hline bul_sc25_2840 & & & \\
\hline bul_sc25_3033 & & & \\
\hline bul_sc25_3046 & & & \\
\hline bul_sc26_0148 & & & \\
\hline bul_sc26_0331 & & & \\
\hline bul_sc26_0332 & & & \\
\hline bul_sc26_0355 & & & \\
\hline bul_sc26_0389 & & & \\
\hline bul_sc26_0390 & & & \\
\hline bul_sc26_0532 & & & \\
\hline bul_sc26_0544 & & & \\
\hline bul_sc26_0565 & & & \\
\hline bul_sc26_0589 & & & \\
\hline bul_sc26_0889 & & & \\
\hline bul_sc26_1225 & & & \\
\hline bul_sc26_1523 & & & \\
\hline bul_sc26_1540 & & & \\
\hline bul_sc26_1856 & & & \\
\hline bul_sc26_1904 & & & \\
\hline bul_sc26_1938 & & & \\
\hline bul_sc26_2023 & & & \\
\hline bul_sc26_2102 & & & \\
\hline bul_sc26_2196 & & & \\
\hline bul_sc26_2198 & & & \\
\hline
\end{tabular}


M. A. T. Groenewegen and J. A. D. L. Blommaert: Mira variables in the bulge, Online Material p 34

Table 2. continued.

\begin{tabular}{|c|c|c|c|}
\hline OGLE-name & Other name & Chemical type & Remarks \\
\hline \multicolumn{4}{|l|}{ bul_sc26_2264 } \\
\hline \multicolumn{4}{|l|}{ bul_sc26_2439 } \\
\hline \multicolumn{4}{|l|}{ bul_sc26_2440 } \\
\hline \multicolumn{4}{|l|}{ bul_sc26_2441 } \\
\hline \multicolumn{4}{|l|}{ bul_sc26_2442 } \\
\hline bul_sc26_2849 & V 2063 & & TO88 \\
\hline \multicolumn{4}{|l|}{ bul_sc26_2859 } \\
\hline \multicolumn{4}{|l|}{ bul_sc26_2864 } \\
\hline bul_sc26_3038 & V 2071 & & TO88 \\
\hline \multicolumn{4}{|l|}{ bul_sc26_3350 } \\
\hline \multicolumn{4}{|l|}{ bul_sc26_3354 } \\
\hline \multicolumn{4}{|l|}{ bul_sc26_3355 } \\
\hline \multicolumn{4}{|l|}{ bul_sc26_3357 } \\
\hline bul_sc26_4174 & V 2084 & & TO88 \\
\hline \multicolumn{4}{|l|}{ bul_sc26_4389 } \\
\hline \multicolumn{4}{|l|}{ bul_sc26_4435 } \\
\hline \multicolumn{4}{|l|}{ bul_sc26_4582 } \\
\hline \multicolumn{4}{|l|}{ bul_sc26_4672 } \\
\hline \multicolumn{4}{|l|}{ bul_sc27_0038 } \\
\hline \multicolumn{4}{|l|}{ bul_sc27_0532 } \\
\hline bul_sc27_0784 & & & \\
\hline bul_sc27_0893 & & & \\
\hline bul_sc27_0977 & IRAS_17445-3520 & & \\
\hline bul_sc27_1070 & & & \\
\hline bul_sc27_1108 & & & \\
\hline bul_sc27_1318 & & & \\
\hline bul_sc27_1335 & & & \\
\hline bul_sc27_1337 & & & \\
\hline bul_sc27_1357 & & & \\
\hline bul_sc27_1703 & & & \\
\hline bul_sc27_1755 & & & \\
\hline bul_sc27_1760 & & & \\
\hline bul_sc27_1950 & & & \\
\hline bul_sc27_1969 & & & \\
\hline bul_sc27_1983 & IRAS_17446-3506 & & \\
\hline bul_sc27_1998 & & & \\
\hline bul_sc27_2013 & & & \\
\hline bul_sc27_2080 & & & \\
\hline bul_sc27_2093 & & & \\
\hline bul_sc27_2133 & & & \\
\hline bul_sc27_2150 & & & \\
\hline bul_sc27_2151 & & & \\
\hline bul_sc27_2152 & & & \\
\hline bul_sc27_2404 & & & \\
\hline bul_sc27_2416 & & & \\
\hline bul_sc27_2417 & & & \\
\hline bul_sc27_2418 & & & \\
\hline bul_sc27_2560 & V 2099 & & TO88 \\
\hline bul_sc27_2693 & & & \\
\hline bul_sc27_2746 & & & \\
\hline bul_sc27_2787 & & & \\
\hline bul_sc27_2857 & & & \\
\hline bul_sc27_2871 & & & \\
\hline bul_sc27_3080 & & & \\
\hline bul_sc27_3094 & & & \\
\hline bul_sc27_3227 & & & \\
\hline bul_sc27_3228 & & & \\
\hline bul_sc27_3245 & & & \\
\hline bul_sc27_3331 & & & \\
\hline
\end{tabular}


M. A. T. Groenewegen and J. A. D. L. Blommaert: Mira variables in the bulge, Online Material p 35

Table 2. continued.

\begin{tabular}{|c|c|c|c|}
\hline OGLE-name & Other name & Chemical type & "Remarks \\
\hline \multicolumn{4}{|l|}{ bul_sc27_3547 } \\
\hline \multicolumn{4}{|l|}{ bul_sc27_3556 } \\
\hline \multicolumn{4}{|l|}{ bul_sc27_3567 } \\
\hline \multicolumn{4}{|l|}{ bul_sc27_3677 } \\
\hline \multicolumn{4}{|l|}{ bul_sc27_3682 } \\
\hline \multicolumn{4}{|l|}{ bul_sc28_0235 } \\
\hline \multicolumn{4}{|l|}{ bul_sc28_0297 } \\
\hline \multicolumn{4}{|l|}{ bul_sc28_0298 } \\
\hline \multicolumn{4}{|l|}{ bul_sc28_0328 } \\
\hline \multicolumn{4}{|l|}{ bul_sc28_0572 } \\
\hline \multicolumn{4}{|l|}{ bul_sc28_0727 } \\
\hline \multicolumn{4}{|l|}{ bul_sc28_0738 } \\
\hline \multicolumn{4}{|l|}{ bul_sc28_0770 } \\
\hline \multicolumn{4}{|l|}{ bul_sc28_0950 } \\
\hline bul_sc28_0991 & IRAS_17431-3654 & & $\begin{array}{l}\text { Kwok et al. (1997), } \\
\text { te Lintel Hekkert (1991) }\end{array}$ \\
\hline \multicolumn{4}{|l|}{ bul_sc28_1058 } \\
\hline \multicolumn{4}{|l|}{ bul_sc28_1073 } \\
\hline \multicolumn{4}{|l|}{ bul_sc28_1201 } \\
\hline \multicolumn{4}{|l|}{ bul_sc28_1206 } \\
\hline bul_sc28_1289 & & & \\
\hline bul_sc28_1449 & & & \\
\hline bul_sc29_0482 & IRAS_17452-3721 & & \\
\hline bul_sc29_0489 & & & \\
\hline bul_sc29_0511 & & & \\
\hline bul_sc29_0534 & & & \\
\hline bul_sc29_0536 & & & \\
\hline bul_sc29_0541 & & & \\
\hline bul_sc29_0546 & & & \\
\hline bul_sc29_0817 & & & \\
\hline bul_sc29_1064 & & & \\
\hline bul_sc29_1241 & & & \\
\hline bul_sc29_1268 & & & \\
\hline bul_sc29_1375 & & & \\
\hline bul_sc29_1487 & & & \\
\hline bul_sc29_1490 & & & \\
\hline bul_sc29_1541 & & & \\
\hline bul_sc29_1542 & & & \\
\hline bul_sc29_1561 & & & \\
\hline bul_sc29_2085 & & & \\
\hline bul_sc29_2088 & & & \\
\hline bul_sc29_2098 & & & \\
\hline bul_sc29_2103 & & & \\
\hline bul_sc29_2117 & & & \\
\hline bul_sc29_2147 & & & \\
\hline bul_sc29_2152 & & & \\
\hline bul_sc29_2159 & & & \\
\hline bul_sc29_2161 & & & \\
\hline bul_sc29_2166 & & & \\
\hline bul_sc29_2179 & & & \\
\hline bul_sc29_2184 & & & \\
\hline bul_sc29_2189 & & & \\
\hline bul_sc29_2254 & & & \\
\hline bul_sc29_2256 & & & \\
\hline bul_sc29_2262 & & & \\
\hline bul_sc29_2266 & & & \\
\hline bul_sc29_2317 & & & \\
\hline bul_sc29_2319 & & & \\
\hline bul_sc30_0213 & & & \\
\hline
\end{tabular}


M. A. T. Groenewegen and J. A. D. L. Blommaert: Mira variables in the bulge, Online Material p 36

Table 2. continued.

\begin{tabular}{|c|c|c|c|}
\hline \multirow{2}{*}{\multicolumn{4}{|c|}{$\frac{\text { OGLE-name }}{\text { bul_sc30_0346 }}$}} \\
\hline & & & \\
\hline \multicolumn{4}{|l|}{ bul_sc30_0630 } \\
\hline \multicolumn{4}{|l|}{ bul_sc30_0707 } \\
\hline \multicolumn{4}{|l|}{ bul_sc30_0827 } \\
\hline \multicolumn{4}{|l|}{ bul_sc30_0868 } \\
\hline \multicolumn{4}{|l|}{ bul_sc30_1085 } \\
\hline \multicolumn{4}{|l|}{ bul_sc30_1110 } \\
\hline \multicolumn{4}{|l|}{ bul_sc30_1156 } \\
\hline \multicolumn{4}{|l|}{ bul_sc30_1174 } \\
\hline \multicolumn{4}{|l|}{ bul_sc30_1305 } \\
\hline \multicolumn{4}{|l|}{ bul_sc30_1341 } \\
\hline \multicolumn{4}{|l|}{ bul_sc30_1486 } \\
\hline \multicolumn{4}{|l|}{ bul_sc30_1627 } \\
\hline \multicolumn{4}{|l|}{ bul_sc30_1821 } \\
\hline \multicolumn{4}{|l|}{ bul_sc30_1977 } \\
\hline \multicolumn{4}{|l|}{ bul_sc30_2143 } \\
\hline \multicolumn{4}{|l|}{ bul_sc30_2377 } \\
\hline bul_sc30_2635 & & & \\
\hline bul_sc30_2788 & & & \\
\hline bul_sc30_2943 & & & \\
\hline bul_sc30_3018 & & & \\
\hline bul_sc30_3086 & & & \\
\hline bul_sc30_3209 & & & \\
\hline bul_sc30_3276 & IRAS_17586-2850 & & \\
\hline bul_sc30_3484 & & & \\
\hline bul_sc30_3510 & & & \\
\hline bul_sc30_3525 & & & \\
\hline bul_sc30_3585 & & & \\
\hline bul_sc30_3654 & & & \\
\hline bul_sc30_3831 & & & \\
\hline bul_sc30_4185 & & & \\
\hline bul_sc30_4213 & & & \\
\hline bul_sc30_4446 & & & \\
\hline bul_sc30_4589 & & & \\
\hline bul_sc30_5019 & & & \\
\hline bul_sc30_5022 & & & \\
\hline bul_sc30_5063 & & & \\
\hline bul_sc30_5077 & & & \\
\hline bul_sc30_5325 & & & \\
\hline bul_sc30_5413 & & & \\
\hline bul_sc30_5442 & & & \\
\hline bul_sc30_5838 & & & \\
\hline bul_sc30_6043 & & & \\
\hline bul_sc30_6096 & & & \\
\hline bul_sc30_6263 & & & \\
\hline bul_sc30_6264 & & & \\
\hline bul_sc30_6402 & & & \\
\hline bul_sc30_6547 & & & \\
\hline bul_sc30_6861 & & & \\
\hline bul_sc31_0109 & & & \\
\hline bul_sc31_0150 & & & \\
\hline bul_sc31_0155 & & & \\
\hline bul_sc31_0246 & & & \\
\hline bul_sc31_0278 & & & \\
\hline bul_sc31_0329 & & & \\
\hline bul_sc31_0463 & & & \\
\hline bul_sc31_0465 & & & \\
\hline bul_sc31_0476 & IRAS_17590-2858 & & \\
\hline bul_sc31_0508 & & & \\
\hline
\end{tabular}


M. A. T. Groenewegen and J. A. D. L. Blommaert: Mira variables in the bulge, Online Material p 37

Table 2. continued.

\begin{tabular}{|c|c|c|c|}
\hline OGLE-name & Other name & Chemical type & Remarks \\
\hline \multicolumn{4}{|l|}{ bul_sc31_0564 } \\
\hline \multicolumn{4}{|l|}{ bul_sc31_0696 } \\
\hline \multicolumn{4}{|l|}{ bul_sc31_0705 } \\
\hline \multicolumn{4}{|l|}{ bul_sc31_0712 } \\
\hline \multicolumn{4}{|l|}{ bul_sc31_0788 } \\
\hline \multicolumn{4}{|l|}{ bul_sc31_0793 } \\
\hline \multicolumn{4}{|l|}{ bul_sc31_0831 } \\
\hline bul_sc31_0856 & SCHB 271 & & Sevenster et al. (1997) \\
\hline \multicolumn{4}{|l|}{ bul_sc31_0866 } \\
\hline \multicolumn{4}{|l|}{ bul_sc31_0868 } \\
\hline \multicolumn{4}{|l|}{ bul_sc31_1043 } \\
\hline \multicolumn{4}{|l|}{ bul_sc31_1138 } \\
\hline \multicolumn{4}{|l|}{ bul_sc31_1235 } \\
\hline \multicolumn{4}{|l|}{ bul_sc31_1296 } \\
\hline \multicolumn{4}{|l|}{ bul_sc31_1308 } \\
\hline \multicolumn{4}{|l|}{ bul_sc31_1315 } \\
\hline \multicolumn{4}{|l|}{ bul_sc31_1360 } \\
\hline \multicolumn{4}{|l|}{ bul_sc31_1407 } \\
\hline bul_sc31_1421 & & & \\
\hline bul_sc31_1581 & & & \\
\hline bul_sc31_1797 & & & \\
\hline bul_sc31_1818 & & & \\
\hline bul_sc31_1865 & & & \\
\hline bul_sc31_2002 & & & \\
\hline bul_sc31_2106 & & & \\
\hline bul_sc31_2161 & & & \\
\hline bul_sc31_2187 & & & \\
\hline bul_sc31_3173 & & & \\
\hline bul_sc31_3271 & & & \\
\hline bul_sc31_3478 & & & \\
\hline bul_sc31_3759 & & & \\
\hline bul_sc31_3956 & & & \\
\hline bul_sc31_3976 & & & \\
\hline bul_sc31_4101 & & & \\
\hline bul_sc31_4128 & & & \\
\hline bul_sc31_4159 & & & \\
\hline bul_sc31_4161 & & & \\
\hline bul_sc31_4167 & & & \\
\hline bul_sc31_4193 & & & \\
\hline bul_sc31_4201 & & & \\
\hline bul_sc31_4208 & & & \\
\hline bul_sc31_4257 & & & \\
\hline bul_sc31_4338 & & & \\
\hline bul_sc31_4384 & & & \\
\hline bul_sc31_4403 & & & \\
\hline bul_sc31_4523 & & & \\
\hline bul_sc31_4527 & & & \\
\hline bul_sc31_4542 & & & \\
\hline bul_sc31_4574 & & & \\
\hline bul_sc32_0016 & & & \\
\hline bul_sc32_0062 & & & \\
\hline bul_sc32_0143 & & & \\
\hline bul_sc32_0317 & & & \\
\hline bul_sc32_0324 & & & \\
\hline bul_sc32_0346 & & & \\
\hline bul_sc32_0448 & & & \\
\hline bul_sc32_0455 & & & \\
\hline bul_sc32_0965 & & & \\
\hline bul_sc32_0969 & & & \\
\hline
\end{tabular}


M. A. T. Groenewegen and J. A. D. L. Blommaert: Mira variables in the bulge, Online Material $p 38$

Table 2. continued.

\begin{tabular}{|c|c|c|c|}
\hline OGLE-name & Other name & Chemical type & Remarks \\
\hline bul_sc32_0980 & & & \\
\hline bul_sc32_1148 & & & \\
\hline bul_sc32_1572 & & & \\
\hline bul_sc32_1573 & & & \\
\hline bul_sc32_1634 & & & \\
\hline bul_sc32_1844 & & & \\
\hline bul_sc32_1889 & & & \\
\hline bul_sc32_2140 & & & \\
\hline bul_sc32_2240 & & & \\
\hline bul_sc32_2293 & & & \\
\hline bul_sc32_2351 & & & \\
\hline bul_sc32_2352 & & & \\
\hline bul_sc32_2359 & & & \\
\hline bul_sc32_2397 & & & \\
\hline bul_sc32_2415 & & & \\
\hline bul_sc32_2652 & & & \\
\hline bul_sc32_2653 & & & \\
\hline bul_sc32_2733 & & & \\
\hline bul_sc32_2734 & & & \\
\hline bul_sc32_2811 & & & \\
\hline bul_sc32_3032 & & & \\
\hline bul_sc32_3067 & & & \\
\hline bul_sc32_3194 & & & \\
\hline bul_sc32_3217 & & & \\
\hline bul_sc32_3222 & & & \\
\hline bul_sc32_3225 & & & \\
\hline bul_sc32_3233 & & & \\
\hline bul_sc32_3236 & & & \\
\hline bul_sc32_3518 & IRAS_18004-2826 & & \\
\hline bul_sc32_3617 & & & \\
\hline bul_sc32_3618 & & & \\
\hline bul_sc32_3762 & & & \\
\hline bul_sc32_3847 & & & \\
\hline bul_sc32_3907 & & & \\
\hline bul_sc32_3953 & & & \\
\hline bul_sc32_4136 & & & \\
\hline bul_sc32_4258 & & & \\
\hline bul_sc32_4349 & & & \\
\hline bul_sc32_4360 & & & \\
\hline bul_sc32_4384 & & & \\
\hline bul_sc32_4613 & & & \\
\hline bul_sc32_4657 & & & \\
\hline bul_sc32_4815 & & & \\
\hline bul_sc32_4967 & & & \\
\hline bul_sc33_0357 & & & \\
\hline bul_sc33_0445 & & & \\
\hline bul_sc33_0478 & & & \\
\hline bul_sc33_0571 & IRAS_18018-2912 & & \\
\hline bul_sc33_0695 & & & \\
\hline bul_sc33_0705 & & & \\
\hline bul_sc33_0743 & & & \\
\hline bul_sc33_0754 & & & \\
\hline bul_sc33_0755 & & & \\
\hline bul_sc33_0979 & & & \\
\hline bul_sc33_1139 & & & \\
\hline bul_sc33_1159 & & & \\
\hline bul_sc33_1178 & & & \\
\hline bul_sc33_1186 & & & \\
\hline bul_sc33_1187 & & & \\
\hline
\end{tabular}


M. A. T. Groenewegen and J. A. D. L. Blommaert: Mira variables in the bulge, Online Material p 39

Table 2. continued.

\begin{tabular}{|c|c|c|c|}
\hline OGLE-name & Other name & Chemical type & Remarks \\
\hline bul_sc33_1229 & & & \\
\hline bul_sc33_1308 & & & \\
\hline bul_sc33_1312 & & & \\
\hline bul_sc33_1439 & & & \\
\hline bul_sc33_1525 & & & \\
\hline bul_sc33_1575 & & & \\
\hline bul_sc33_1584 & & & \\
\hline bul_sc33_1603 & & & \\
\hline bul_sc33_1611 & & & \\
\hline bul_sc33_1654 & & & \\
\hline bul_sc33_1795 & & & \\
\hline bul_sc33_1796 & & & \\
\hline bul_sc33_1877 & & & \\
\hline bul_sc33_1882 & & & \\
\hline bul_sc33_1954 & IRAS_18026-2856 & & \\
\hline bul_sc33_1998 & & & \\
\hline bul_sc33_2139 & & & \\
\hline bul_sc33_2194 & & & \\
\hline bul_sc33_2208 & & & \\
\hline bul_sc33_2244 & & & \\
\hline bul_sc33_2249 & & & \\
\hline bul_sc33_2334 & & & \\
\hline bul_sc33_2339 & & & \\
\hline bul_sc33_2343 & & & \\
\hline bul_sc33_2357 & & & \\
\hline bul_sc33_2358 & & & \\
\hline bul_sc33_2360 & & & \\
\hline bul_sc33_2361 & & & \\
\hline bul_sc33_2362 & & & \\
\hline bul_sc33_2367 & & & \\
\hline bul_sc33_2496 & & & \\
\hline bul_sc33_2514 & & & \\
\hline bul_sc33_2517 & & & \\
\hline bul_sc33_2608 & & & \\
\hline bul_sc33_2656 & & & \\
\hline bul_sc33_2672 & & & \\
\hline bul_sc33_2678 & & & \\
\hline bul_sc33_2679 & & & \\
\hline bul_sc33_2681 & & & \\
\hline bul_sc33_2682 & & & \\
\hline bul_sc33_2683 & & & \\
\hline bul_sc33_2685 & & & \\
\hline bul_sc33_2767 & & & \\
\hline bul_sc33_2898 & & & \\
\hline bul_sc33_2902 & & & \\
\hline bul_sc33_2907 & & & \\
\hline bul_sc33_2908 & & & \\
\hline bul_sc33_3079 & & & \\
\hline bul_sc33_3080 & & & \\
\hline bul_sc33_3089 & & & \\
\hline bul_sc33_3091 & & & \\
\hline bul_sc33_3095 & & & \\
\hline bul_sc33_3099 & & & \\
\hline bul_sc33_3102 & & & \\
\hline bul_sc33_3104 & & & \\
\hline bul_sc33_3127 & & & \\
\hline bul_sc33_3139 & & & \\
\hline bul_sc33_3140 & & & \\
\hline bul_sc33_3162 & & & \\
\hline
\end{tabular}


M. A. T. Groenewegen and J. A. D. L. Blommaert: Mira variables in the bulge, Online Material p 40

Table 2. continued.

\begin{tabular}{|c|c|c|c|}
\hline OGLE-name & Other name & Chemical type & Remarks \\
\hline \multicolumn{4}{|l|}{ bul_sc33_3176 } \\
\hline \multicolumn{4}{|l|}{ bul_sc33_3178 } \\
\hline \multicolumn{4}{|l|}{ bul_sc33_3179 } \\
\hline \multicolumn{4}{|l|}{ bul_sc33_3180 } \\
\hline \multicolumn{4}{|l|}{ bul_sc33_3182 } \\
\hline \multicolumn{4}{|l|}{ bul_sc33_3240 } \\
\hline \multicolumn{4}{|l|}{ bul_sc33_3250 } \\
\hline \multicolumn{4}{|l|}{ bul_sc33_3251 } \\
\hline \multicolumn{4}{|l|}{ bul_sc33 3266} \\
\hline \multicolumn{4}{|l|}{ bul_sc33_3268 } \\
\hline \multicolumn{4}{|l|}{ bul_sc33_3349 } \\
\hline \multicolumn{4}{|l|}{ bul_sc33_3350 } \\
\hline \multicolumn{4}{|l|}{ bul_sc33_3383 } \\
\hline \multicolumn{4}{|l|}{ bul_sc33_3395 } \\
\hline \multicolumn{4}{|l|}{ bul_sc33_3398 } \\
\hline \multicolumn{4}{|l|}{ bul_sc33_3399 } \\
\hline \multicolumn{4}{|l|}{ bul_sc33_3409 } \\
\hline bul_sc33_3414 & & & \\
\hline bul_sc33_3438 & & & \\
\hline bul_sc33_3699 & & & \\
\hline bul_sc33_3737 & & & \\
\hline bul_sc33_3755 & & & \\
\hline bul_sc33_3922 & & & \\
\hline bul_sc33_4149 & & & \\
\hline bul_sc33_4161 & & & \\
\hline bul_sc33_4189 & & & \\
\hline bul_sc33_4268 & & & \\
\hline bul_sc33_4445 & & & \\
\hline bul_sc33_4508 & & & \\
\hline bul_sc33_4566 & & & \\
\hline bul_sc33_4571 & & & \\
\hline bul_sc34_0243 & & & \\
\hline bul_sc34_0816 & & & \\
\hline bul_sc34_0905 & & & \\
\hline bul_sc34_0982 & & & \\
\hline bul_sc34_1635 & & & \\
\hline bul_sc34_1643 & & & \\
\hline bul_sc34_1664 & & & \\
\hline bul_sc34_1677 & & & \\
\hline bul_sc34_1754 & & & \\
\hline bul_sc34_1945 & & & \\
\hline bul_sc34_2181 & & & \\
\hline bul_sc34_2335 & & & \\
\hline bul_sc34_2343 & & & \\
\hline bul_sc34_2647 & & & \\
\hline bul_sc34_3242 & IRAS_17547-2909 & & \\
\hline bul_sc34_3269 & & & \\
\hline bul_sc34_3759 & ABC01_113.18155.43, & & Glass et al. (1999), \\
\hline & OOS03-PJ175843.9-290711, & & GWCF, LE76 \\
\hline & ISOGAL-PJ175843.9-290711, & & \\
\hline & TLE_Sgr_I_65, V3972 Sgr & & \\
\hline bul_sc34_3854 & & & \\
\hline bul_sc34_4084 & & & \\
\hline bul_sc34_4090 & & & \\
\hline bul_sc34_4100 & & & \\
\hline bul_sc34_4159 & & & \\
\hline bul_sc34_4218 & & & \\
\hline bul_sc34_4327 & & & \\
\hline bul_sc34_4336 & & & \\
\hline
\end{tabular}


M. A. T. Groenewegen and J. A. D. L. Blommaert: Mira variables in the bulge, Online Material p 41

Table 2. continued.

\begin{tabular}{|c|c|c|c|}
\hline OGLE-name & Other name & Chemical type & $\overline{\text { Remarks }}$ \\
\hline bul_sc34_4509 & & & \\
\hline bul_sc34_4516 & & & \\
\hline bul sc34 4552 & & & \\
\hline bul_sc34_4559 & & & \\
\hline bul_sc34_4573 & & & \\
\hline bul_sc34_4586 & ISOGAL-PJ175840.2-290122 & & \\
\hline bul_sc34_4603 & & & \\
\hline bul_sc34_5012 & & & \\
\hline bul_sc34_5028 & & & \\
\hline bul_sc34_5217 & & & \\
\hline bul_sc34_5218 & & & \\
\hline bul_sc34_5343 & & & \\
\hline bul_sc34_5405 & IRAS_17546-2854 & & \\
\hline bul_sc34_5448 & & & \\
\hline bul_sc34_5475 & & & \\
\hline bul_sc34_5624 & & & \\
\hline bul_sc34_5863 & & & \\
\hline bul_sc34_6276 & & & \\
\hline bul_sc34_6342 & & & \\
\hline bul_sc34_6360 & & & \\
\hline bul_sc34_6362 & & & \\
\hline bul_sc34_6386 & & & \\
\hline bul_sc34_6747 & & & \\
\hline bul_sc34_7087 & & & \\
\hline bul_sc34_7122 & & & \\
\hline bul_sc34_7153 & & & \\
\hline bul_sc34_7428 & & & \\
\hline bul_sc34_7629 & & & \\
\hline bul_sc34_7639 & & & \\
\hline bul_sc34_7685 & & & \\
\hline bul_sc34_7694 & & & \\
\hline bul_sc34_7708 & & & \\
\hline bul_sc34_7744 & & & \\
\hline bul_sc34_7761 & & & \\
\hline bul_sc34_7879 & & & \\
\hline bul_sc35_0492 & & & \\
\hline bul_sc35_0898 & & & \\
\hline bul_sc35_0967 & & & \\
\hline bul_sc35_1052 & & & \\
\hline bul_sc35_1175 & IRAS_18008-2810 & & \\
\hline bul_sc35_1373 & & & \\
\hline bul_sc35_1376 & & & \\
\hline bul_sc35_1395 & & & \\
\hline bul_sc35_1426 & & & \\
\hline bul_sc35_1531 & & & \\
\hline bul_sc35_1620 & & & \\
\hline bul_sc35_1654 & & & \\
\hline bul_sc35_1779 & & & \\
\hline bul_sc35_1782 & & & \\
\hline bul_sc35_1786 & & & \\
\hline bul_sc35_2687 & & & \\
\hline bul_sc35_2842 & & & \\
\hline bul_sc35_2932 & & & \\
\hline bul_sc35_2935 & & & \\
\hline bul_sc35_2965 & & & \\
\hline bul_sc35_3024 & & & \\
\hline bul_sc35_3365 & & & \\
\hline bul_sc35_3388 & & & \\
\hline bul_sc35_3668 & & & \\
\hline
\end{tabular}


Table 2. continued.

\begin{tabular}{|c|c|c|c|}
\hline \multirow{2}{*}{$\begin{array}{l}\text { OGLE-name } \\
\text { bul_sc35_3693 }\end{array}$} & Other name & Chemical type & Remarks \\
\hline & & & \\
\hline bul_sc35_3768 & & & \\
\hline bul_sc35_3797 & & & \\
\hline bul_sc35_3851 & & & \\
\hline bul_sc35_3879 & & & \\
\hline bul_sc35_4087 & & & \\
\hline bul_sc35_4353 & & & \\
\hline bul_sc35_4385 & & & \\
\hline bul_sc35_4475 & & & \\
\hline bul_sc35_4538 & & & \\
\hline bul_sc35_4778 & & & \\
\hline bul_sc35_4952 & & & \\
\hline bul_sc35_5075 & & & \\
\hline bul_sc35_5078 & & & \\
\hline bul_sc35_5091 & & & \\
\hline bul_sc36_0030 & & & \\
\hline bul_sc36_0320 & & & \\
\hline bul_sc36_0812 & & & \\
\hline bul_sc36_1128 & & & \\
\hline bul_sc36_1243 & IRAS_18028-2817, ESO 456-51 & M8.5 & $\begin{array}{l}\text { Munari \& Zwitter (2002), Kohoutek (2002), } \\
\text { Medina Tanco \& Steiner (1995) }\end{array}$ \\
\hline bul_sc36_1254 & & & \\
\hline bul_sc36_1301 & & & \\
\hline bul_sc36_1571 & & & \\
\hline bul_sc36_1654 & & & \\
\hline bul_sc36_2158 & & & \\
\hline bul_sc36_2650 & & & \\
\hline bul_sc36_2764 & & & \\
\hline bul_sc36_2803 & & & \\
\hline bul_sc36_3014 & & & \\
\hline bul_sc36_3646 & & & \\
\hline bul_sc36_3690 & & & \\
\hline bul_sc36_3894 & & & \\
\hline bul_sc36_3908 & & & \\
\hline bul_sc36_3985 & & & \\
\hline bul_sc36_3990 & & & \\
\hline bul_sc36_4282 & & & \\
\hline bul_sc36_4779 & & & \\
\hline bul_sc36_4985 & & & \\
\hline bul_sc36_5032 & & & \\
\hline bul_sc36_5181 & & & \\
\hline bul_sc36_5594 & & & \\
\hline bul_sc36_5774 & & & \\
\hline bul_sc36_5794 & & & \\
\hline bul_sc36_5953 & & & \\
\hline bul_sc36_6354 & & & \\
\hline bul_sc36_6977 & & & \\
\hline bul_sc36_7454 & & & \\
\hline bul_sc36_7668 & & & \\
\hline bul_sc36_7676 & & & \\
\hline bul_sc36_7687 & & & \\
\hline bul_sc36_8151 & & & \\
\hline bul_sc36_8350 & & & \\
\hline bul_sc36_8625 & & & \\
\hline bul_sc37_0066 & & & \\
\hline bul_sc37_0090 & & & \\
\hline bul_sc37_0222 & & & \\
\hline bul_sc37_0617 & & & \\
\hline bul_sc37_0637 & & & \\
\hline
\end{tabular}


M. A. T. Groenewegen and J. A. D. L. Blommaert: Mira variables in the bulge, Online Material p 43

Table 2. continued.

\begin{tabular}{|c|c|c|c|}
\hline OGLE-name & Other name & Chemical type & $\overline{\text { Remarks }}$ \\
\hline bul_sc37_0770 & & & \\
\hline bul_sc37_0807 & & & \\
\hline bul sc37 0812 & & & \\
\hline bul_sc37_0832 & & & \\
\hline bul_sc37_0881 & & & \\
\hline bul_sc37_0914 & & & \\
\hline bul_sc37_0916 & & & \\
\hline bul_sc37_0931 & & & \\
\hline bul_sc37_1102 & & & \\
\hline bul_sc37_1105 & & & \\
\hline bul_sc37_1154 & & & \\
\hline bul_sc37_1176 & & & \\
\hline bul_sc37_1253 & & & \\
\hline bul_sc37_1353 & & & \\
\hline bul_sc37_1452 & & & \\
\hline bul_sc37_1465 & & & \\
\hline bul_sc37_1468 & & & \\
\hline bul_sc37_1508 & & & \\
\hline bul_sc37_1535 & & & \\
\hline bul_sc37_1551 & & & \\
\hline bul_sc37_1656 & & & \\
\hline bul_sc37_1666 & & & \\
\hline bul_sc37_1678 & & & \\
\hline bul_sc37_1779 & & & \\
\hline bul_sc37_1925 & & & \\
\hline bul_sc37_1975 & & & \\
\hline bul_sc37_2000 & & & \\
\hline bul_sc37_2025 & & & \\
\hline bul_sc37_2428 & & & \\
\hline bul_sc37_2433 & & & \\
\hline bul_sc37_2473 & & & \\
\hline bul_sc37_2474 & & & \\
\hline bul_sc37_2676 & & & \\
\hline bul_sc37_2726 & & & \\
\hline bul_sc37_2776 & & & \\
\hline bul_sc37_2787 & & & \\
\hline bul_sc37_2843 & & & \\
\hline bul_sc37_2937 & IRAS_17497-3004 & & \\
\hline bul_sc37_2946 & & & \\
\hline bul_sc37_2959 & & & \\
\hline bul_sc37_3015 & & & \\
\hline bul_sc37_3422 & & & \\
\hline bul_sc37_3454 & & & \\
\hline bul_sc37_3575 & & & \\
\hline bul_sc37_3634 & ISOGAL-PJ175247.9-295841 & & \\
\hline bul_sc37_3723 & & & \\
\hline bul_sc37_3761 & & & \\
\hline bul_sc37_3803 & & & \\
\hline bul_sc37_3807 & & & \\
\hline bul_sc37_3843 & & & \\
\hline bul_sc37_3857 & & & \\
\hline bul_sc37_3952 & & & \\
\hline bul_sc37_4220 & & & \\
\hline bul_sc37_4272 & & & \\
\hline bul_sc37_4334 & & & \\
\hline bul_sc37_4519 & & & \\
\hline bul_sc37_4583 & & & \\
\hline bul_sc37_4594 & & & \\
\hline bul_sc37_4613 & & & \\
\hline
\end{tabular}


M. A. T. Groenewegen and J. A. D. L. Blommaert: Mira variables in the bulge, Online Material p 44

Table 2. continued.

\begin{tabular}{|c|c|c|c|}
\hline OGLE-name & Other name & Chemical type & $\overline{\text { Remarks }}$ \\
\hline bul_sc37_4614 & & & \\
\hline bul_sc37_4656 & & & \\
\hline bul_sc37_4681 & & & \\
\hline bul_sc37_4701 & & & \\
\hline bul_sc37_4737 & & & \\
\hline bul_sc37_4790 & & & \\
\hline bul_sc37_4860 & & & \\
\hline bul_sc37_5111 & & & \\
\hline bul_sc37_5123 & & & \\
\hline bul_sc37_5173 & & & \\
\hline bul_sc37_5187 & & & \\
\hline bul_sc37_5261 & & & \\
\hline bul_sc37_5276 & & & \\
\hline bul_sc37_5335 & & & \\
\hline bul_sc37_5358 & & & \\
\hline bul_sc37_5466 & & & \\
\hline bul_sc37_5561 & & & \\
\hline bul_sc37_5562 & & & \\
\hline bul_sc37_5569 & & & \\
\hline bul_sc37_5602 & & & \\
\hline bul_sc37_5707 & & & \\
\hline bul_sc37_5816 & & & \\
\hline bul_sc37_5926 & & & \\
\hline bul_sc37_6103 & & & \\
\hline bul_sc37_6185 & & & \\
\hline bul_sc37_6373 & & & \\
\hline bul_sc37_6380 & & & \\
\hline bul_sc37_6467 & & & \\
\hline bul_sc37_6578 & & & \\
\hline bul_sc37_7067 & & & \\
\hline bul_sc37_7089 & & & \\
\hline bul_sc37_7108 & & & \\
\hline bul_sc37_7120 & & & \\
\hline bul_sc37_7122 & & & \\
\hline bul_sc37_7265 & & & \\
\hline bul_sc37_7408 & & & \\
\hline bul_sc37_7553 & & & \\
\hline bul_sc37_7622 & & & \\
\hline bul_sc37_7628 & & & \\
\hline bul_sc37_7637 & & & \\
\hline bul_sc37_7900 & & & \\
\hline bul_sc37_7905 & & & \\
\hline bul_sc37_8065 & & & \\
\hline bul_sc37_8204 & & & \\
\hline bul_sc38_0013 & & & \\
\hline bul_sc38_0489 & & & \\
\hline bul_sc38_0595 & & & \\
\hline bul_sc38_0596 & & & \\
\hline bul_sc38_0665 & & & \\
\hline bul_sc38_0673 & & & \\
\hline bul_sc38_0847 & & & \\
\hline bul_sc38_0953 & & & \\
\hline bul_sc38_1127 & & & \\
\hline bul_sc38_1143 & & & \\
\hline bul_sc38_1155 & & & \\
\hline bul_sc38_1156 & & & \\
\hline bul_sc38_1170 & & & \\
\hline bul_sc38_1208 & & & \\
\hline bul_sc38_1213 & & & \\
\hline
\end{tabular}


M. A. T. Groenewegen and J. A. D. L. Blommaert: Mira variables in the bulge, Online Material p 45

Table 2. continued.

\begin{tabular}{|c|c|c|c|}
\hline OGLE-name & Other name & Chemical type & Remarks \\
\hline bul_sc38_1502 & & & \\
\hline bul_sc38_1505 & & & \\
\hline bul_sc38_1738 & & & \\
\hline bul_sc38_1783 & & & \\
\hline bul_sc38_1796 & & & \\
\hline bul_sc38_1815 & V4707 Sgr & & \\
\hline bul_sc38_1921 & & & \\
\hline bul_sc38_1923 & & & \\
\hline bul_sc38_1933 & & & \\
\hline bul_sc38_1940 & & & \\
\hline bul_sc38_1996 & & & \\
\hline bul_sc38_2018 & & & \\
\hline bul_sc38_2284 & & & \\
\hline bul_sc38_2460 & & & \\
\hline bul_sc38_2464 & & & \\
\hline bul_sc38_2649 & & & \\
\hline bul_sc38_2737 & & & \\
\hline bul_sc38_2739 & & & \\
\hline bul_sc38_2802 & & & \\
\hline bul_sc38_2811 & & & \\
\hline bul_sc38_3090 & & & \\
\hline bul_sc38_3101 & & & \\
\hline bul_sc38_3421 & & & \\
\hline bul_sc38_3450 & & & \\
\hline bul_sc38_3711 & & & \\
\hline bul_sc38_4092 & & & \\
\hline bul_sc38_4234 & & & \\
\hline bul_sc38_4384 & & & \\
\hline bul_sc38_4440 & & & \\
\hline bul_sc38_4460 & & & \\
\hline bul_sc38_4469 & & & \\
\hline bul_sc38_4474 & & & \\
\hline bul_sc38_4481 & & & \\
\hline bul_sc38_4636 & & & \\
\hline bul_sc38_4733 & & & \\
\hline bul_sc38_4793 & & & \\
\hline bul_sc38_4881 & & & \\
\hline bul_sc38_4923 & & & \\
\hline bul_sc38_4955 & & & \\
\hline bul_sc38_5002 & & & \\
\hline bul_sc38_5005 & & & \\
\hline bul_sc38_5060 & & & \\
\hline bul_sc39_0024 & & & \\
\hline bul_sc39_0051 & & & \\
\hline bul_sc39_0076 & & & \\
\hline bul_sc39_0229 & & & \\
\hline bul_sc39_0315 & & & \\
\hline bul_sc39_0342 & & & \\
\hline bul_sc39_0372 & & & \\
\hline bul_sc39_0498 & & & \\
\hline bul_sc39_0513 & & & \\
\hline bul_sc39_0713 & & & \\
\hline bul_sc39_1153 & & & \\
\hline bul_sc39_1166 & & & \\
\hline bul_sc39_1333 & & & \\
\hline bul_sc39_1537 & & & \\
\hline bul_sc39_1593 & IRAS_17523-2959 & & \\
\hline bul_sc39_1606 & & & \\
\hline
\end{tabular}


M. A. T. Groenewegen and J. A. D. L. Blommaert: Mira variables in the bulge, Online Material p 46

Table 2. continued.

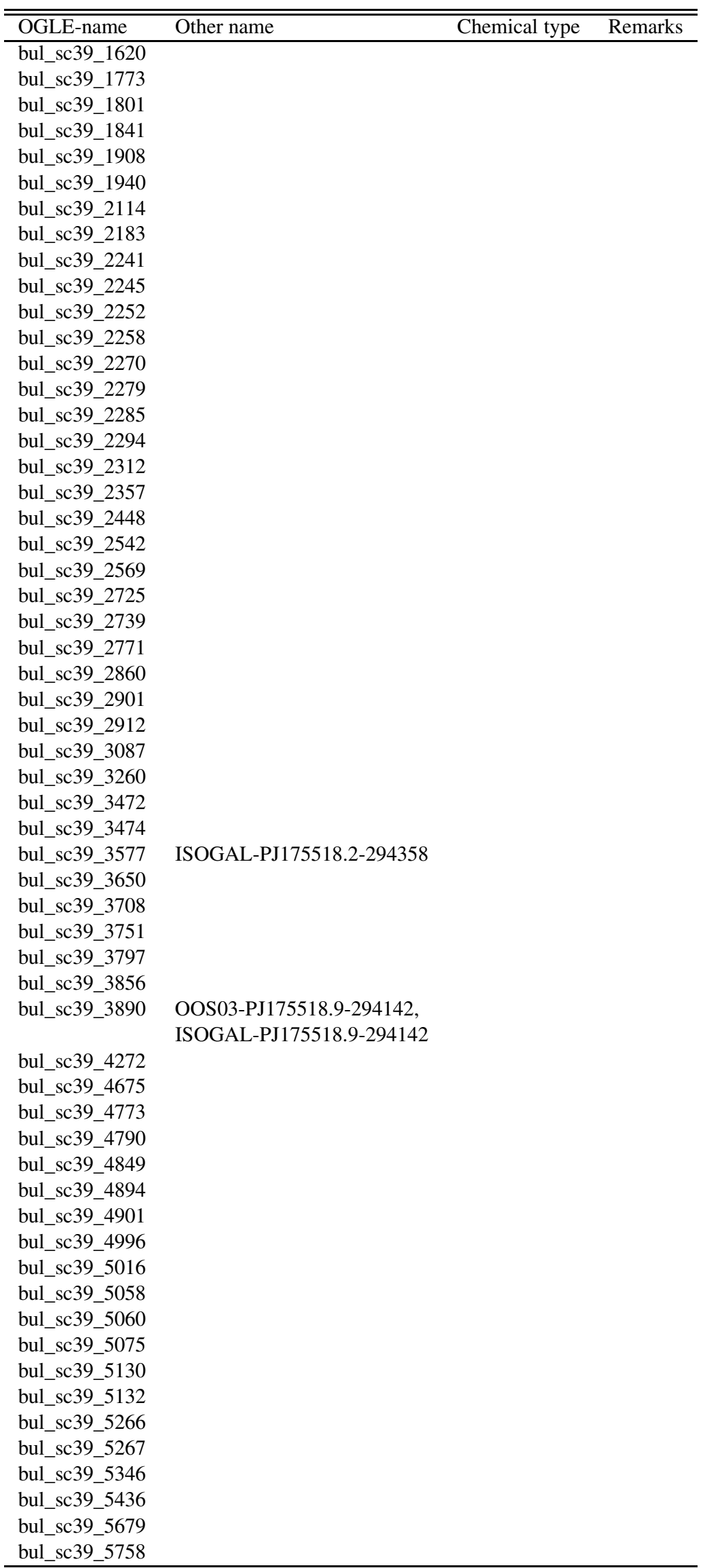


M. A. T. Groenewegen and J. A. D. L. Blommaert: Mira variables in the bulge, Online Material p 47

Table 2. continued.

\begin{tabular}{|c|c|c|c|}
\hline OGLE-name & Other name & Chemical type & Remarks \\
\hline bul_sc39_5762 & & & \\
\hline bul_sc39 5816 & & & \\
\hline bul sc39 5851 & & & \\
\hline bul_sc39_5995 & & & \\
\hline bul_sc39_6025 & & & \\
\hline bul_sc39_6108 & & & \\
\hline bul_sc39 6256 & & & \\
\hline bul_sc39_6259 & & & \\
\hline bul_sc39_6294 & & & \\
\hline bul_sc39_6369 & & & \\
\hline bul_sc39_6385 & & & \\
\hline bul_sc39_6392 & & & \\
\hline bul_sc39_6577 & & & \\
\hline bul_sc39_6596 & & & \\
\hline bul_sc39_6679 & & & \\
\hline bul_sc39_6721 & & & \\
\hline bul_sc39_6798 & & & \\
\hline bul_sc39_6816 & & & \\
\hline bul_sc39_6950 & & & \\
\hline bul_sc39_7047 & & & \\
\hline bul_sc39_7050 & & & \\
\hline bul_sc39_7058 & & & \\
\hline bul_sc39_7081 & & & \\
\hline bul_sc39_7086 & & & \\
\hline bul_sc39 7235 & & & \\
\hline bul_sc40_0068 & & & \\
\hline bul_sc40_0148 & & & \\
\hline bul_sc40_0170 & & & \\
\hline bul_sc40_0280 & & & \\
\hline bul_sc40_0281 & V2199 & & TO88 \\
\hline bul_sc40_0292 & & & \\
\hline bul_sc40_0356 & & & \\
\hline bul_sc40_0408 & & & \\
\hline bul_sc40_0425 & & & \\
\hline bul_sc40_0529 & V2177 & & TO88 \\
\hline bul_sc40_0554 & & & \\
\hline bul_sc40_0681 & & & \\
\hline bul_sc40_0704 & & & \\
\hline bul_sc40_0753 & & & \\
\hline bul_sc40_0763 & & & \\
\hline bul_sc40_0846 & & & \\
\hline bul_sc40_0904 & & & \\
\hline bul_sc40_1028 & & & \\
\hline bul_sc40_1155 & & & \\
\hline bul_sc40_1215 & & & \\
\hline bul_sc40_1299 & & & \\
\hline bul_sc40_1408 & & & \\
\hline bul_sc40_1603 & & & \\
\hline bul_sc40_1674 & & & \\
\hline bul_sc40_1720 & & & \\
\hline bul_sc40_1726 & & & \\
\hline bul_sc40_1738 & & & \\
\hline bul_sc40_1785 & & & \\
\hline bul_sc40_1858 & & & \\
\hline bul_sc40_1953 & & & \\
\hline bul_sc40_2086 & & & \\
\hline bul_sc40_2353 & & & \\
\hline bul_sc40_2401 & & & \\
\hline bul_sc40_2423 & & & \\
\hline
\end{tabular}


M. A. T. Groenewegen and J. A. D. L. Blommaert: Mira variables in the bulge, Online Material p 48

Table 2. continued.

\begin{tabular}{|c|c|c|c|}
\hline \multirow{2}{*}{$\begin{array}{l}\text { OGLE-name } \\
\text { bul_sc40_2086 }\end{array}$} & Other name & Chemical type & Remarks \\
\hline & & & \\
\hline bul_sc40_2353 & & & \\
\hline bul_sc40_2401 & & & \\
\hline bul_sc40_2423 & & & \\
\hline bul_sc40_2444 & & & \\
\hline bul_sc40_2652 & & & \\
\hline bul_sc40_2653 & & & \\
\hline bul_sc40_2735 & V 2203 & & TO88 \\
\hline bul_sc40_2750 & & & \\
\hline bul_sc40_2751 & & & \\
\hline bul_sc40_2853 & & & \\
\hline bul_sc40_2936 & & & \\
\hline bul_sc40_3033 & V 2198 & & TO88 \\
\hline bul_sc40_3035 & & & \\
\hline bul_sc40_3091 & IRAS_17476-3300 & & \\
\hline bul_sc40_3098 & & & \\
\hline bul_sc40_3149 & & & \\
\hline bul_sc40_3220 & & & \\
\hline bul_sc40_3524 & & & \\
\hline bul_sc40_3687 & & & \\
\hline bul_sc40_3694 & & & \\
\hline bul_sc40_3795 & & & \\
\hline bul_sc40_3854 & & & \\
\hline bul_sc40_3863 & & & \\
\hline bul_sc40_3962 & & & \\
\hline bul_sc41_0191 & & & \\
\hline bul_sc41_0367 & & & \\
\hline bul_sc41_0551 & & & \\
\hline bul_sc41_0553 & & & \\
\hline bul_sc41_0649 & & & \\
\hline bul_sc41_0837 & & & \\
\hline bul_sc41_0957 & & & \\
\hline bul_sc41_1345 & & & \\
\hline bul_sc41_1357 & & & \\
\hline bul_sc41_1462 & & & \\
\hline bul_sc41_1497 & & & \\
\hline bul_sc41_1569 & & & \\
\hline bul_sc41_1621 & & & \\
\hline bul_sc41_1634 & & & \\
\hline bul_sc41_1636 & & & \\
\hline bul_sc41_1637 & & & \\
\hline bul_sc41_1977 & & & \\
\hline bul_sc41_2039 & & & \\
\hline bul_sc41_2065 & & & \\
\hline bul_sc41_2219 & & & \\
\hline bul_sc41_2384 & & & \\
\hline bul_sc41_2457 & & & \\
\hline bul_sc41_2517 & & & \\
\hline bul_sc41_2648 & & & \\
\hline bul_sc41_2714 & & & \\
\hline bul_sc41_2738 & & & \\
\hline bul_sc41_2756 & & & \\
\hline bul_sc41_2782 & & & \\
\hline bul_sc41_2786 & & & \\
\hline bul_sc41_2870 & & & \\
\hline bul_sc41_2885 & & & \\
\hline bul_sc41_2961 & & & \\
\hline bul_sc41_3028 & & & \\
\hline bul_sc41_3089 & & & \\
\hline
\end{tabular}


M. A. T. Groenewegen and J. A. D. L. Blommaert: Mira variables in the bulge, Online Material p 49

Table 2. continued.

\begin{tabular}{|c|c|c|c|}
\hline OGLE-name & Other name & Chemical type & Remarks \\
\hline \multicolumn{4}{|l|}{ bul_sc41_3094 } \\
\hline \multicolumn{4}{|l|}{ bul_sc41_3231 } \\
\hline \multicolumn{4}{|l|}{ bul_sc41_3279 } \\
\hline \multicolumn{4}{|l|}{ bul_sc41_3304 } \\
\hline \multicolumn{4}{|l|}{ bul_sc41_3319 } \\
\hline \multicolumn{4}{|l|}{ bul_sc41_3366 } \\
\hline \multicolumn{4}{|l|}{ bul_sc41_3443 } \\
\hline \multicolumn{4}{|l|}{ bul_sc41_3458 } \\
\hline \multicolumn{4}{|l|}{ bul_sc41_3512 } \\
\hline \multicolumn{4}{|l|}{ bul_sc411_3536 } \\
\hline \multicolumn{4}{|l|}{ bul_sc41_3623 } \\
\hline \multicolumn{4}{|l|}{ bul_sc41_3672 } \\
\hline \multicolumn{4}{|l|}{ bul_sc41_3897 } \\
\hline \multicolumn{4}{|l|}{ bul_sc41_3898 } \\
\hline \multicolumn{4}{|l|}{ bul_sc41_3911 } \\
\hline \multirow{2}{*}{\multicolumn{4}{|c|}{$\begin{array}{l}\text { bui_sc42_0039 } \\
\text { bul_sc42_0046 }\end{array}$}} \\
\hline & & & \\
\hline \multicolumn{4}{|l|}{ bul_sc42_0047 } \\
\hline bul_sc42_0048 & & & \\
\hline bul_sc42_0084 & & & \\
\hline bul_sc42_0272 & & & \\
\hline bul_sc42_0356 & & & \\
\hline bul_sc42_0362 & & & \\
\hline bul_sc42_0363 & & & \\
\hline bul_sc42_0365 & & & \\
\hline bul_sc42_0391 & & & \\
\hline bul_sc42_0756 & & & \\
\hline bul_sc42_0826 & & & \\
\hline bul_sc42_0945 & & & \\
\hline bul_sc42_0967 & & & \\
\hline bul_sc42_0969 & & & \\
\hline bul_sc42_1312 & & & \\
\hline bul_sc42_2002 & & & \\
\hline bul_sc42_2179 & & & \\
\hline bul_sc42_2190 & & & \\
\hline bul_sc42_2342 & & & \\
\hline bul_sc42_2356 & & & \\
\hline bul_sc42_2357 & & & \\
\hline bul_sc42_2418 & & & \\
\hline bul_sc42_2528 & & & \\
\hline bul_sc42_2600 & & & \\
\hline bul_sc42_3184 & & & \\
\hline bul_sc42_3473 & & & \\
\hline bul_sc42_3485 & & & \\
\hline bul_sc42_3488 & & & \\
\hline bul_sc42_4183 & & & \\
\hline bul_sc42_4185 & & & \\
\hline bul_sc43_0078 & & & \\
\hline bul_sc43_0097 & & & \\
\hline bul_sc43_0105 & & & \\
\hline bul_sc43_0115 & & & \\
\hline bul_sc43_0150 & V 2603 & & Terzan \& Gosset (1991; TG91) \\
\hline bul_sc43_0152 & & & \\
\hline bul_sc43_0153 & & & \\
\hline bul_sc43_0175 & & & \\
\hline bul_sc43_0199 & & & \\
\hline bul_sc43_0243 & & & \\
\hline bul_sc43_0244 & V 2590 & & TG91 \\
\hline bul_sc43_0313 & & & \\
\hline
\end{tabular}


M. A. T. Groenewegen and J. A. D. L. Blommaert: Mira variables in the bulge, Online Material p 50

Table 2. continued.

\begin{tabular}{|c|c|c|c|}
\hline OGLE-name & Other name & Chemical type & Remarks \\
\hline bul_sc43_0358 & V 2632 & & TG91 \\
\hline bul_sc43_0376 & V 2662 & & TG91 \\
\hline bul_sc43_0398 & & & \\
\hline bul_sc43_0433 & V 2655 & & TG91 \\
\hline bul_sc43_0441 & & & \\
\hline bul_sc43_0442 & & & \\
\hline bul_sc43_0513 & IRAS_17322-2726 & & \\
\hline bul_sc43_0571 & & & \\
\hline bul_sc43_0575 & V 2652 & & TG91 \\
\hline bul_sc43_0582 & V 2665 & & TG91 \\
\hline bul_sc43_0592 & & & \\
\hline bul_sc43_0661 & IRAS_17322-2724 & & \\
\hline bul_sc43_0765 & V 2642 & & TG91 \\
\hline bul_sc43_0769 & & & \\
\hline bul_sc43_0843 & V 2613 & & TG91 \\
\hline bul_sc43_0865 & & & \\
\hline bul_sc43_0879 & & & \\
\hline bul_sc43_0882 & & & \\
\hline bul_sc43_0896 & & & \\
\hline bul_sc43_0918 & & & \\
\hline bul_sc43_1114 & & & \\
\hline bul_sc43_1115 & & & \\
\hline bul_sc43_1116 & & & \\
\hline bul_sc43_1117 & & & \\
\hline bul_sc43_1120 & & & \\
\hline bul_sc43_1127 & & & \\
\hline bul_sc43_1143 & & & \\
\hline bul_sc43_1149 & & & \\
\hline bul_sc43_1153 & & & \\
\hline bul_sc43_1154 & & & \\
\hline bul_sc43_1156 & & & \\
\hline bul_sc43_1157 & & & \\
\hline bul_sc43_1159 & & & \\
\hline bul_sc43_1168 & & & \\
\hline bul_sc43_1170 & & & \\
\hline bul_sc43_1171 & & & \\
\hline bul_sc43_1172 & & & \\
\hline bul_sc43_1174 & & & \\
\hline bul_sc43_1175 & & & \\
\hline bul_sc43_1176 & & & \\
\hline bul_sc43_1177 & & & \\
\hline bul_sc43_1178 & & & \\
\hline bul_sc43_1213 & V 2644 & & TG91 \\
\hline bul_sc43_1215 & & & \\
\hline bul_sc43_1216 & & & \\
\hline bul_sc43_1217 & & & \\
\hline bul_sc43_1223 & & & \\
\hline bul_sc43_1246 & & & \\
\hline bul_sc43_1247 & & & \\
\hline bul_sc43_1255 & & & \\
\hline bul_sc43_1257 & & & \\
\hline bul_sc43_1258 & & & \\
\hline bul_sc43_1273 & & & \\
\hline bul_sc43_1274 & & & \\
\hline bul_sc43_1275 & & & \\
\hline bul_sc43_1279 & & & \\
\hline bul_sc43_1281 & & & \\
\hline bul_sc43_1282 & & & \\
\hline bul_sc43_1283 & & & \\
\hline
\end{tabular}


M. A. T. Groenewegen and J. A. D. L. Blommaert: Mira variables in the bulge, Online Material p 51

Table 2. continued.

\begin{tabular}{|c|c|c|c|}
\hline OGLE-name & Other name & Chemical type & $\overline{\text { Remarks }}$ \\
\hline \multicolumn{4}{|l|}{ bul_sc43_1286 } \\
\hline \multicolumn{4}{|l|}{ bul_sc43_1300 } \\
\hline \multicolumn{4}{|l|}{ bul_sc43_1319 } \\
\hline \multicolumn{4}{|l|}{ bul_sc43_1485 } \\
\hline bul_sc43_1492 & V 2633 & & TG91 \\
\hline bul_sc43_1519 & V 2598 & & TG91 \\
\hline \multicolumn{4}{|l|}{ bul_sc43_1524 } \\
\hline bul_sc43_1579 & V 2588 & & TG91 \\
\hline bul_sc433_1667 & V 2639 & & TG91 \\
\hline \multicolumn{4}{|l|}{ bul_sc43_1676 } \\
\hline bul_sc43_1715 & V 2615 & & TG91 \\
\hline \multicolumn{4}{|l|}{ bul_sc43_1919 } \\
\hline \multicolumn{4}{|l|}{ bul sc43 2034} \\
\hline \multicolumn{4}{|l|}{ bul_sc43_2071 } \\
\hline \multicolumn{4}{|l|}{ bul_sc43_2098 } \\
\hline \multicolumn{4}{|l|}{ bul_sc43_2118 } \\
\hline \multicolumn{4}{|l|}{ bul_sc43_2149 } \\
\hline \multicolumn{4}{|l|}{ bul_sc43_2173 } \\
\hline \multicolumn{4}{|l|}{ bul_sc43_2214 } \\
\hline bul_sc43_2263 & V 2659 & & TG91 \\
\hline bul_sc43_2295 & V 2624 & & TG91 \\
\hline \multicolumn{4}{|l|}{ bul_sc43_2333 } \\
\hline \multicolumn{4}{|l|}{ bul_sc43_2338 } \\
\hline \multicolumn{4}{|l|}{ bul_sc43_2407 } \\
\hline bul_sc433_2423 & & & \\
\hline bul_sc43_2461 & V 2611 & & TG91 \\
\hline bul_sc43_2627 & V 2643 & & TG91 \\
\hline bul_sc43_2761 & & & \\
\hline bul_sc43_2782 & & & \\
\hline bul_sc43_2793 & & & \\
\hline bul_sc43_2857 & V 2596 & & TG91 \\
\hline bul_sc43_2860 & & & \\
\hline bul_sc43_2935 & & & \\
\hline bul_sc43_3001 & V 2629 & & TG91 \\
\hline bul_sc43_3015 & & & \\
\hline bul_sc43_3029 & & & \\
\hline bul_sc43_3071 & & & \\
\hline bul_sc43_3142 & V 2595 & & TG91 \\
\hline bul_sc43_3240 & & & \\
\hline bul_sc43_3321 & & & \\
\hline bul_sc43_3348 & & & \\
\hline bul_sc44_0054 & V 2128 & & TO88 \\
\hline bul_sc44_0237 & & & \\
\hline bul_sc44_0377 & & & \\
\hline bul_sc44_0581 & & & \\
\hline bul_sc44_0606 & & & \\
\hline bul_sc44_0755 & & & \\
\hline bul_sc44_0792 & & & \\
\hline bul_sc44_0795 & & & \\
\hline bul_sc44_0796 & & & \\
\hline bul_sc44_0856 & & & \\
\hline bul_sc44_0913 & & & \\
\hline bul_sc44_0968 & & & \\
\hline bul_sc44_0980 & & & \\
\hline bul_sc44_1144 & & & \\
\hline bul_sc44_1237 & & & \\
\hline bul_sc44_1272 & & & \\
\hline bul_sc44_1352 & & & \\
\hline bul_sc44_1357 & & & \\
\hline
\end{tabular}


M. A. T. Groenewegen and J. A. D. L. Blommaert: Mira variables in the bulge, Online Material p 52

Table 2. continued.

\begin{tabular}{|c|c|c|c|}
\hline \multirow{2}{*}{\multicolumn{4}{|c|}{$\frac{\text { OGLE-name }}{\text { bul_sc44_1373 }}$}} \\
\hline & & & \\
\hline \multicolumn{4}{|l|}{ bul_sc44_1498 } \\
\hline \multicolumn{4}{|l|}{ bul_sc44_1568 } \\
\hline \multicolumn{4}{|l|}{ bul_sc44_1667 } \\
\hline \multicolumn{4}{|l|}{ bul_sc44_1722 } \\
\hline \multicolumn{4}{|l|}{ bul_sc44_1727 } \\
\hline \multicolumn{4}{|l|}{$\begin{array}{l}\text { bul_sc44_1748 } \\
\text { bul_sc44_1893 }\end{array}$} \\
\hline \multicolumn{4}{|l|}{ bul_sc44_1893 } \\
\hline \multicolumn{4}{|l|}{ bul_sc44_2025 } \\
\hline \multicolumn{4}{|l|}{ bul_sc44_2164 } \\
\hline \multicolumn{4}{|l|}{ bul_sc44_2189 } \\
\hline \multicolumn{4}{|l|}{ bul_sc44_2257 } \\
\hline \multicolumn{4}{|l|}{ bul_sc44_2277 } \\
\hline \multicolumn{4}{|l|}{ bul_sc44_2288 } \\
\hline \multicolumn{4}{|l|}{ bul_sc44_2301 } \\
\hline \multicolumn{4}{|l|}{ bul_sc44_2316 } \\
\hline \multicolumn{4}{|l|}{ bul_sc44_2317 } \\
\hline bul_sc44_2319 & & & \\
\hline bul_sc44_2351 & & & \\
\hline bul_sc44_2361 & & & \\
\hline bul_sc44_2391 & & & \\
\hline bul_sc44_2478 & & & \\
\hline bul_sc44_2493 & & & \\
\hline bul_sc44_2644 & & & \\
\hline bul_sc444_2659 & & & \\
\hline bul_sc44_2665 & & & \\
\hline bul_sc44_2925 & & & \\
\hline bul_sc44_2991 & & & \\
\hline bul_sc44_2999 & & & \\
\hline bul_sc44_3021 & & & \\
\hline bul_sc44_3028 & & & \\
\hline bul_sc44_3053 & & & \\
\hline bul_sc44_3085 & & & \\
\hline bul_sc44_3089 & & & \\
\hline bul_sc44_3128 & & & \\
\hline bul_sc44_3136 & & & \\
\hline bul_sc44_3232 & & & \\
\hline bul_sc44_3268 & & & \\
\hline bul_sc44_3286 & & & \\
\hline bul_sc44_3298 & & & \\
\hline bul_sc44_3393 & IRAS_17463-3001 & & \\
\hline bul_sc44_3694 & & & \\
\hline bul_sc44_3721 & & & \\
\hline bul_sc44_3729 & & & \\
\hline bul_sc44_3885 & & & \\
\hline bul_sc44_4178 & & & \\
\hline bul_sc44_4354 & & & \\
\hline bul_sc44_4364 & & & \\
\hline bul_sc44_4436 & & & \\
\hline bul_sc44_4548 & & & \\
\hline bul_sc44_4608 & & & \\
\hline bul_sc44_4689 & & & \\
\hline bul_sc44_4743 & & & \\
\hline bul_sc44_4786 & & & \\
\hline bul_sc44_4898 & & & \\
\hline bul_sc44_4939 & & & \\
\hline bul_sc44_4940 & & & \\
\hline bul_sc44_4942 & & & \\
\hline bul_sc44_5103 & & & \\
\hline
\end{tabular}


M. A. T. Groenewegen and J. A. D. L. Blommaert: Mira variables in the bulge, Online Material p 53

Table 2. continued.

\begin{tabular}{|c|c|c|c|}
\hline \multirow{2}{*}{$\begin{array}{l}\text { OGLE-name } \\
\text { bul_sc44_5111 }\end{array}$} & \multirow[t]{2}{*}{ Other name } & \multirow[t]{2}{*}{ Chemical type } & \multirow[t]{2}{*}{ Remarks } \\
\hline & & & \\
\hline bul_sc44_5142 & & & \\
\hline bul_sc44_5290 & & & \\
\hline bul_sc44_5347 & & & \\
\hline bul_sc44_5372 & & & \\
\hline bul_sc44_5391 & & & \\
\hline bul_sc44_5411 & & & \\
\hline bul_sc44_5412 & & & \\
\hline bul_sc44_5460 & & & \\
\hline bul_sc44_5472 & & & \\
\hline bul_sc44_5506 & & & \\
\hline bul_sc44_5534 & & & \\
\hline bul_sc44_5577 & & & \\
\hline bul_sc44_5578 & & & \\
\hline bul_sc44_5596 & & & \\
\hline bul_sc44_5643 & & & \\
\hline bul_sc44_5685 & & & \\
\hline bul_sc44_5726 & & & \\
\hline bul_sc44_5813 & & & \\
\hline bul_sc44_5974 & IRAS_17463-2946 & M8 & Raharto et al. (1984) \\
\hline bul_sc44_5975 & & & \\
\hline bul_sc44_5993 & & & \\
\hline bul_sc44_6016 & & & \\
\hline bul_sc44_6061 & & & \\
\hline bul_sc44_6153 & & & \\
\hline bul_sc44_6158 & & & \\
\hline bul_sc44_6159 & & & \\
\hline bul_sc44_6160 & & & \\
\hline bul_sc44_6195 & & & \\
\hline bul_sc44_6276 & & & \\
\hline bul_sc44_6279 & & & \\
\hline bul_sc44_6300 & & & \\
\hline bul_sc44_6352 & & & \\
\hline bul_sc44_6484 & & & \\
\hline bul_sc44_6529 & & & \\
\hline bul_sc44_6531 & & & \\
\hline bul_sc44_6554 & & & \\
\hline bul_sc44_6641 & & & \\
\hline bul_sc44_6644 & & & \\
\hline bul_sc44_6708 & & & \\
\hline bul_sc44_6859 & & & \\
\hline bul_sc44_7060 & & & \\
\hline bul_sc44_7107 & & & \\
\hline bul_sc44_7301 & & & \\
\hline bul_sc44_7317 & & & \\
\hline bul_sc44_7324 & & & \\
\hline bul_sc44_7450 & & & \\
\hline bul_sc44_7473 & & & \\
\hline bul_sc44_7551 & & & \\
\hline bul_sc444_7626 & & & \\
\hline bul_sc44_7649 & & & \\
\hline bul_sc44_7690 & & & \\
\hline bul_sc44_7738 & & & \\
\hline bul_sc44_7777 & & & \\
\hline bul_sc44_7828 & & & \\
\hline bul_sc45_0148 & & & \\
\hline bul_sc45_0164 & & & \\
\hline bul_sc45_0170 & & & \\
\hline bul_sc45_0179 & & & \\
\hline
\end{tabular}


Table 2. continued.

\begin{tabular}{|c|c|c|c|}
\hline OGLE-name & Other name & Chemical type & Remarks \\
\hline $\begin{array}{l}\text { bul_sc45_0226 } \\
\text { bul_sc45_0230 } \\
\text { bul_sc45_0275 } \\
\text { bul_sc45_0360 } \\
\text { bul_sc45_0369 } \\
\text { bul_sc45_0373 } \\
\text { bul_sc45_0418 } \\
\text { bul_sc45_0546 }\end{array}$ & & & . \\
\hline bul_sc45_0704 & $\begin{array}{l}\text { BW6_V4_MISC, TLE_NGC_6522_644, } \\
\text { BMB_87, IRAS_18001-3012, } \\
\text { MACHO 119.20088.3574 }\end{array}$ & M8 & $\begin{array}{l}\text { GS03, Frogel \& Whitford (1987; hereafter FW87), } \\
\text { G86, GF82, LE76 }\end{array}$ \\
\hline $\begin{array}{l}\text { bul_sc45_0799 } \\
\text { bul_sc45_0842 } \\
\text { bul_sc45_0961 } \\
\text { bul_sc45_0962 } \\
\text { bul_sc45_0972 }\end{array}$ & & & \\
\hline $\begin{array}{l}\text { bul_sc45_1068 } \\
\text { bul_sc45_1316 }\end{array}$ & $\begin{array}{l}\text { OOS03-PJ180313.8-300306, } \\
\text { ISOGAL-PJ180313.8-300306, } \\
\text { TLE_NGC_6522_395, BMB_68, } \\
\text { MACHO 119.20091.28, V1414 Sgr } \\
\text { OOS03-PJ180311.5-295747, } \\
\text { ISOGAL-PJ180311.5-295747 }\end{array}$ & M6 & GS03, LE76 \\
\hline $\begin{array}{l}\text { bul_sc45_1323 } \\
\text { bul_sc45_1374 } \\
\text { bul_sc45_1490 }\end{array}$ & & & \\
\hline bul_sc45_1586 & $\begin{array}{l}\text { TLE_NGC_6522_721, } \\
\text { BMB_220, V1471 Sgr, } \\
\text { BMB_220, V1471 Sgr, } \\
\text { MACHO 119.20353.109 }\end{array}$ & M6 & GS03, GF82, LE76 \\
\hline bul_sc45_1589 & IRAS_18007-2951, BMB_250 & M7 & FW87 \\
\hline $\begin{array}{l}\text { bul_sc45_1713 } \\
\text { bul_sc45_1725 } \\
\text { bul_sc45_1941 } \\
\text { bul_sc45_1980 } \\
\text { bul_sc45_1998 } \\
\text { bul_sc45_2020 } \\
\text { bul_sc45_2055 } \\
\text { bul_sc46_0025 } \\
\text { bul_sc46_0125 } \\
\text { bul_sc46_0214 } \\
\text { bul_sc46_0225 } \\
\text { bul_sc46_0242 } \\
\text { bul_sc46_0254 } \\
\text { bul_sc46_0347 } \\
\text { bul_sc46_0355 } \\
\text { bul_sc46_0427 } \\
\text { bul_sc46_0490 }\end{array}$ & BMB_238 & M6.5 & \\
\hline bul_sc46_0866 & $\begin{array}{l}\text { TLE_NGC_6522_786, } \\
\text { BMB_285, V 1502 Sgr, } \\
\text { MACHO } 119.20610 .46\end{array}$ & & GS03, LE76 \\
\hline $\begin{array}{l}\text { bul_sc46_0950 } \\
\text { bul_sc46_0996 } \\
\text { bul_sc46_1026 }\end{array}$ & ISOGAL-PJ180448.4-300400 & & \\
\hline $\begin{array}{l}\text { bul_sc46_1048 } \\
\text { bul_sc46_1115 } \\
\text { bul_sc46_1117 } \\
\text { bul_sc46_1163 }\end{array}$ & $\begin{array}{l}\text { ISOGAL-PJ180441.9-295958, } \\
\text { BW7_V2_MISC }\end{array}$ & & \\
\hline
\end{tabular}


M. A. T. Groenewegen and J. A. D. L. Blommaert: Mira variables in the bulge, Online Material p 55

Table 2. continued.

\begin{tabular}{l} 
OGLE-name \\
\hline Other name \\
bul_sc46_1245 \\
bul_sc46_1378 \\
bul_sc46_1419 \\
bul_sc46_1467 \\
bul_sc46_1474 \\
bul_sc46_1583 \\
bul_sc46_1735 \\
bul_sc46_2021 \\
bul_sc47_0024 \\
bul_sc47_0125 \\
bul_sc47_0180 \\
bul_sc47_0303 \\
bul_sc47_0456 \\
bul_sc47_0489 \\
bul_sc47_0655 \\
bul_sc47_0758 \\
bul_sc47_0816 \\
bul_sc47_0964 \\
bul_sc47_1000 \\
bul_sc47_1090 \\
bul_sc48_0186 \\
bul_sc48_0230 \\
bul_sc48_0280 \\
bul_sc48_0306 IRAS_17248-3953 \\
bul_sc48_0316 \\
bul_sc48_0340 \\
bul_sc48_0438 \\
bul_sc48_0465 \\
bul_sc48_0568 \\
bul_sc48_0573 \\
bul_sc48_0773 \\
bul_sc48_0914 \\
bul_sc49_0157 \\
bul_sc49_0249 \\
bul_sc49_0251 \\
bul_sc49_0262 \\
bul_sc49_0280 \\
bul_sc49_0463 \\
bul_sc49_0477 \\
bul_sc49_0526 \\
bul_sc49_0575 \\
bul_sc49_0744 \\
bul_sc49_0754 \\
bul_sc49_0756 \\
bul_sc49_0757 \\
bul_sc49_0759 \\
bul_sc49_0770 \\
bul_sc49_0778 \\
bul_sc49_0779 \\
bul_sc49_0782 \\
\hline
\end{tabular}

